\title{
DETERMINACY FROM STRONG REFLECTION
}

\author{
JOHN STEEL AND STUART ZOBLE \\ SEPTEMBER 16, 2008
}

Abstract. The Axiom of Determinacy holds in the inner model $L(\mathbb{R})$ assuming Martin's Maximum for partial orderings of size c.

\section{INTRODUCTION}

A theorem of Neeman gives a particularly elegant sufficient condition for a set $B$ of reals to be determined: $\mathrm{B}$ is determined if there is a triple $(M, \tau, \Sigma)$ which captures $B$ in the sense that $M$ is a model of a sufficient fragment of set theory, $\tau$ is a forcing term in $M$ with respect to the collapse of some Woodin cardinal $\delta$ of $M$ to be countable, and $\Sigma$ is an $\omega+1$-iteration strategy for $M$ such that

$$
B \cap N[g]=i(\tau)_{g},
$$

whenever $i: M \rightarrow N$ is an iteration map by $\Sigma$, and $g$ is generic over $N$ for the collapse of $i(\delta)$. The core model induction, subject of the forthcoming book [16], is a method pioneered by Woodin for constructing such triples $(M, \tau, \Sigma)$ by induction on the complexity of the set $B$. It seems to be the only generally applicable method for making fine consistency strength calculations above the level of one Woodin cardinal. We employ this method here to establish that the Axiom of Determinacy holds in the inner model $L(\mathbb{R})$ from consequences of the maximal forcing axiom $\mathrm{MM}(c)$, or Martin's Maximum for partial orderings of size c. The particular consequences we use are the saturation of the nonstationary ideal on $\omega_{1}$, and the simultaneous reflection principle $\operatorname{WRP}_{(2)}\left(\omega_{2}\right)$ asserting that for any stationary subsets $S$ and $T$ of $\left[\omega_{2}\right]^{\omega}$ there is an ordinal $\delta<\omega_{2}$ so that $S \cap[\delta]^{\omega}$ and $T \cap[\delta]^{\omega}$ are both stationary in $[\delta]^{\omega}$.

Theorem 1. $\operatorname{WRP}_{(2)}\left(\omega_{2}\right)$ plus NS saturated implies $\mathrm{AD}^{L(\mathbb{R})}$.

Corollary 2. $\mathrm{MM}(c)$ implies $\mathrm{AD}^{L(\mathbb{R})}$.

2000 Mathematics Subject Classification. Primary 03E45; Secondary 03E35.

Key words and phrases. Stationary Reflection, Nonstationary Ideal, Determinacy. 
This theorem, obtained in late 2000, builds on Woodin's proof of PD from the same hypotheses (9.85 of [25]), and represents the first proof of the consistency of the Axiom of Determinacy from Forcing Axioms. The first author subsequently obtained the same conclusion in [22] from a single failure of square (and hence from PFA) building on Woodin's theorem that PFA together with an inaccessible gives AD in the Solovay model. Unlike that proof, which relies on covering lemmas to produce the models required for the induction step, we use the generic embedding derived from the saturated ideal. This has its precedents in the first author's proof of $\Delta_{2}^{1}$ determinacy from a presaturated ideal on $\omega_{1}$ together with a measurable cardinal, and in Woodin's proof, via the core model induction, of $\mathrm{AD}^{L(\mathbb{R})}$ from an $\omega_{1}$-dense ideal on $\omega_{1}$.

While our theorem represents the best known lower bound for the consistency strength of $\mathrm{MM}(c)$, this principle is believed to be much stronger. In Chapter 6 we discuss some results suggesting that the arguments here cannot take us much farther than $A D^{L(\mathbb{R})}$, and some extensions of the main theorem which could plausibly yield an equiconsistency result at the level of $\omega^{2}$ Woodin cardinals from modified hypotheses.

Acknowledgement. Some of the arguments presented here have their origin in [26], written under the supervision of Hugh Woodin. The second author would like to thank him for his guidance and support.

\section{FramewORK OF THE INDUCTION}

Let us recall some terminology from [22]

Definition 3. Let $U \subseteq \mathbb{R}$, and $k<\omega$. Let $N$ be countable and transitive, and suppose $\delta_{0}, \ldots, \delta_{k}, S$, and $T$ are such that

(a) $N \models$ ZFC $\wedge \delta_{0}<\ldots<\delta_{k}$ are Woodin cardinals,

(b) $N \models S, T$ are trees which project to complements after the collapse of $\delta_{k}$ to be countable, and

(c) there is an $\omega_{1}+1$-iteration strategy $\Sigma$ for $N$ such that whenever $i: N \rightarrow P$ is an iteration map by $\Sigma$ and $P$ is countable, then $p[i(S)] \subseteq U$ and $p[i(T)] \subseteq \mathbb{R} \backslash U$.

Then we say that $N$ is a coarse $(k, U)$-Woodin mouse, as witnessed by $S, T, \Sigma, \delta_{0}, \ldots, \delta_{k}$.

Definition 4. $W_{\alpha}^{*}$ denotes the following assertion. If $U \subseteq \mathbb{R}$, and there are scales $\vec{\phi}$ and $\vec{\psi}$ on $U$ and $\mathbb{R} \backslash U$ respectively such that $\overrightarrow{\vec{\phi}^{*}}, \overrightarrow{\psi^{*}} \in J_{\alpha}(\mathbb{R})$, where $\vec{\phi}^{*}$ and $\vec{\psi}^{*}$ are the sequences of prewellorders associated to the scales, then for all $k<\omega$ and $x \in \mathbb{R}$ there are $N, \Sigma$ such that 
(1) $x \in N$, and $N$ is a coarse $(k, U)$-Woodin mouse, as witnessed by $\Sigma$, and

(2) $\Sigma \uparrow \mathrm{HC} \in J_{\alpha}(\mathbb{R})$.

Our core model induction will show that

$$
V[g] \models \forall \alpha W_{\alpha}^{*},
$$

whenever $g \subset \operatorname{Col}\left(\omega, \omega_{1}\right)$ is $V$-generic. From $W_{\alpha}^{*}$ we get a version of mouse capturing by fine-structural mice. Let us recall the relevant definitions from [22]. To any $\Sigma_{1}$ formula $\theta(v)$ we associate formulae $\theta^{k}(v)$ for $k \in \omega$, such that $\theta^{k}$ is $\Sigma_{k}$, and for any $\gamma$ and any real $x$,

$$
J_{\gamma+1}(\mathbb{R}) \models \theta[x] \Leftrightarrow \exists k<\omega J_{\gamma}(\mathbb{R}) \models \theta^{k}[x] .
$$

Our fine-structural witnesses are as follows.

Definition 5. Suppose $\theta(v)$ is a $\Sigma_{1}$ formula (in the language of set theory expanded by a name for $\mathbb{R})$, and $\mathrm{z}$ is a real; then a $(\theta, z)$-witnesss is an $\omega$-sound, $\left(\omega, \omega_{1}, \omega_{1}+1\right)$-iterable $z$-mouse $\mathcal{N}$ in which there are $\delta_{0}<\ldots<\delta_{9}, S$, and $T$ such that $\mathcal{N}$ satisfies the formulae expressing

(a) ZFC,

(b) $\delta_{0}, \ldots, \delta_{9}$ are Woodin,

(c) $S$ and $T$ are trees on some $\omega \times \eta$ which are absolutely complementing in $V^{\operatorname{Col}\left(\omega, \delta_{9}\right)}$, and

(d) For some $k<\omega, p[T]$ is the $\Sigma_{k+3}$-theory (in the language with names for each real) of $J_{\gamma}(\mathbb{R})$, where $\gamma$ is least such that $J_{\gamma}(\mathbb{R})=$ $\theta^{k}[z]$.

Definition 6. $W_{\alpha}$ is the assertion: if $\theta(v)$ is $\Sigma_{1}, z \in \mathbb{R}$, and $J_{\alpha}(\mathbb{R})=$ $\theta[z]$, then there is a $(\theta, z)$-witness $\mathcal{N}$ whose associated iteration strategy, when restricted to countable iteration trees, is in $J_{\alpha}(\mathbb{R})$.

We have

Lemma 7. Assume $W_{\alpha}^{*}$ holds; then

(a) $J_{\alpha}(\mathbb{R}) \models \mathrm{AD}$, and

(b) $W_{\alpha}$ holds if $\alpha$ is a limit ordinal.

See [22] for a proof of (b), which is essentially Woodin's mouse set theorem for $L(\mathbb{R})$. Part (a) is an easy exercise for our intended reader. ${ }^{1}$ We note that $W_{\alpha}$ easily implies other forms of capturing by fine-structural mice, and in particular:

\footnotetext{
${ }^{1}$ It follows directly from Neeman's theorem in [12], but one doesn't need that much firepower. The results of Martin-Steel [9], together with Woodin's genericity iterations (see [20]), give it easily.
} 
Lemma 8. Assume $W_{\alpha}$ holds; then if a is countable transitive, and $b \subseteq a, b$ is ordinal definable from parameters in $a \cup\{a\}$ over some $J_{\gamma}(\mathbb{R})$, where $\gamma<\alpha$, then there is a a-premouse $\mathcal{M}$ such that $b \in \mathcal{M}$, and $J_{\alpha}(\mathbb{R})=\mathcal{M}$ is $\omega_{1}$-iterable.

In our proof of $W_{\beta+1}^{*}$, we get the capturing mice we need in $V[g][G]$, where $G \subset\left(P\left(\omega_{1}\right) / N S\right)^{V}$ is generic over $V[g]$. We then use an inductively maintained resemblance between $V[g]$ and $V[g][G]$ to find these mice in $V[g]$. This leads us to a second induction hypothesis.

Definition 9. $I_{\alpha}$ is the assertion: whenever $h \times G$ is $\operatorname{Col}\left(\omega, \omega_{1}\right) \times$ $\left(P\left(\omega_{1}\right) / N S\right)^{V}$-generic over $V$, there is a $\Sigma_{1}$ embeddings

$$
\pi: J_{\alpha}(\mathbb{R})^{V[h]} \rightarrow J_{\alpha}(\mathbb{R})^{V[G][h]}
$$

such that $\pi \uparrow \omega \alpha$ is the identity.

An easy consequence of Lemma $7(\mathrm{a})$ and our induction hypotheses together is

Lemma 10. Assume $I_{\alpha}$ holds Let $g \times G$ be $\operatorname{Col}\left(\omega, \omega_{1}\right) \times\left(P\left(\omega_{1}\right) / N S\right)^{V}$ generic over $V$, and suppose $V[g] \models W_{\alpha}^{*}$; then $\mathrm{AD}$ holds in $J_{\alpha}(\mathbb{R})^{V[g]}$, and in $J_{\alpha}(\mathbb{R})^{V[G][g]}$.

We shall see later that AD holds in $J_{\alpha}(\mathbb{R})^{V[G]}$ as well; see 39 .

As mentioned above, we shall be proving that $W_{\alpha}^{*}$ holds in $V[g]$, by induction on $\alpha$. Clearly, the only stages which matter are the critical ones, where

Definition 11. An ordinal $\beta$ is critical just in case there is some set $U \subseteq \mathbb{R}$ such that $U$ and $\mathbb{R} \backslash U$ admit scales in $J_{\beta+1}(\mathbb{R})$, but $U$ admits no scale in $J_{\beta}(\mathbb{R})$.

Once again, we are identifying a scale with the sequence of its prewellorderings here. Clearly, we need only show that $W_{\beta+1}^{*}$ holds whenever $\beta$ is critical, in order to conclude that $W_{\alpha}^{*}$ holds for all $\alpha$. It follows from [23] that if $\beta$ is critical, then $\beta+1$ is critical. Moreover, if $\beta$ is a limit of critical ordinals, then $\beta$ is critical if and only if $J_{\beta}(\mathbb{R})$ is not an admissible set. Letting $\beta$ be critical, we then have the following possibilities

(1) $\beta=\eta+1$, for some critical $\eta$;

(2) $\beta$ is a limit of critical ordinals, and either

(a) $\operatorname{cof}(\beta)=\omega$, or

(b) $\operatorname{cof}(\beta)>\omega$, but $J_{\beta}(\mathbb{R})$ is not admissible;

(3) $\alpha=\sup (\{\eta<\beta \mid \eta$ is critical $\})$ is such that $\alpha<\beta$, and either

(a) $[\alpha, \beta]$ is a $\Sigma_{1}$ gap, or 
(b) $\beta-1$ exists, and $[\alpha, \beta-1]$ is a $\Sigma_{1}$ gap.

We shall call (3) the admissible case, because it corresponds precisely to crossing a $\Sigma_{1}$ gap whose initial ordinal is admissible.

The hypothesis $I_{\alpha}$ is not used as an input in the arguments of the admissible case but is produced as an output. On the other hand, it is used in the inadmissible case. $I_{\alpha}$ originates in [26], where it is part of a proof that the saturation of NS and $\operatorname{WRP}_{(2)}\left(\omega_{2}\right)$ imply $W_{\alpha}^{*}$ for $\alpha$ the first admissible over the reals. Our argument here follows the overall structure of [26] pretty closely. What we add are some techniques for getting past admissible ordinals using hybrid strategy mice. These techniques were also used in [22].

A final remark on the organization of our proof. It might perhaps be more natural to think of ourselves as proving that $W_{\alpha}^{*}$ holds in $V$, for all $\alpha$. As in any core model induction, given a critical ordinal $\beta$, the first step toward $W_{\beta+1}^{*}$ in $V$ is to find a (hybrid) mouse operator $J$ which codes up truth at the level of the first pointclass $\sum_{\sim}^{J_{\beta}(\mathbb{R})}$ having the scale property. In order to prove $W_{\beta+1}^{*}$, we then need to capture truth over $J_{\beta}(\mathbb{R})$ in full, and for this we need to construct the " $k$ many - $J$-Woodins" operators $M_{k}^{J}$, for all $k$. These "successor steps" are where core model theory (relativised to $J$ ) comes in. The core model theory in our argument requires that $J$ first be extended to $H\left(\omega_{3}\right)$, in a way that is consistent with its images $\pi(J)$ under $N S$ generic ultrapower maps. The extension to $H\left(\omega_{2}\right)$ is equivalent to an extension to $H\left(\omega_{1}\right)^{V[g]}$. It is at this point that we must consider $W_{\gamma}^{*}$ in $V[g]$, where $\gamma<\pi(\beta)$ for some $N S$-generic $\pi$. Extending $J$ involves showing that $\pi(\beta)$ is independent of the $N S$-generic, and $W_{\gamma}^{*}$ and holds in $V[g]$ at all $\gamma \leq \pi(\beta)$. This subinduction in $V[g]$ leading to an extension of $J$ is also where $I_{\gamma}$ is used.

\section{THE SUCCESSOR STEP}

The successor step in a core model induction is the step from a model operator $J$ to the one $J$-Woodin operator. There are two important sorts of model operators for which one needs to make this step, the mouse operators and the hybrid mouse operators. We shall consider only mouse operators in this section, but the proof works without much change for model operators in general. We shall consider hybrid mouse operators in the last section of the paper.

Our proof builds on the proofs of the following theorems.

Theorem 12 (Steel, [18]). Assume there is a measurable cardinal and a presaturated ideal on $\omega_{1}$; then $\Delta_{2}^{1}$ determinacy holds. 
Theorem 13 (Woodin, [25][9.85]). (Woodin) Assume $\mathrm{WRP}_{(2)}\left(\omega_{2}\right)$ and that NS is saturated; then PD holds, and continues to hold in the universe after $\omega_{2}$ is collapsed.

Roughly, the proof of the first theorem supplies the core model theory we need, and the proof of the second shows how to integrate the core model theory into a core model induction.

Woodin's argument shows inductively that $H\left(\omega_{3}\right)$ is closed under the $M_{n}^{\#}$ mouse operator for each $n<\omega$. We shall give the proof in somewhat greater generality. Roughly, rather than doing the first $\omega$ steps of a core model induction, we will be doing the general successor step. For that, we need to have as data an operator $J$ such that $H\left(\omega_{3}\right)$ is closed under $J$; we then show that $H\left(\omega_{3}\right)$ is closed under $M_{1}^{J, \sharp}$. $\left(M_{1}^{\sharp}(a)=M_{1}^{J \sharp}(a)\right.$, for $J(\mathcal{P})=\operatorname{rud}(\mathcal{P})$.) Here we shall just consider the case that $J$ is a first order mouse operator. In the last section we shall be forced to consider more general $J$.

Since it requires only a little additional work, we shall assume only $\operatorname{WRP}_{(2)}\left(\omega_{2}\right)$, there is a presaturated ideal on $\omega_{1}$, and $2^{\omega_{1}} \leq \omega_{2}$. Woodin has shown that $\operatorname{WRP}_{(2)}\left(\omega_{2}\right)$ and the saturation of NS together imply that $2^{\omega_{1}} \leq \omega_{2}$; see 30 below. We believe that in fact $\operatorname{WRP}_{(2)}\left(\omega_{2}\right)$ together with a presaturated ideal on $\omega_{1}$ should be enough for our argument, but have not checked that carefully. See 31 below.

The properties of our initial mouse operator $J$ that make the general successor step possible are that it

(1) $J$ condenses well,

(2) $J$ relativises well,

(3) $J$ determines itself on generic extensions, and

(4) $I$-generic embeddings move $J$ to itself.

Here $I$ is our presaturated ideal. We now explain these properties. The reader should keep in mind the example $J_{n}(b)=M_{n}^{\sharp}(b)$, which has all of them. That it has property (4) is one of the main things our induction will show.

We need to consider premice over some transitive set $b$, with a distinguished parameter $a \in b$. In this context, we shall always in this paper assume that $b$ is selfwellordered, that is, equipped with a wellorder that is (uniformly over all $b$ under consideration) rudimentary in $a .^{2}$ The language $\mathcal{L}_{0}$ of such relativized premice is the language of premice, together with an additional constant symbols $\dot{b}$ and $\dot{a}$ for the set thrown in at the bottom and its distinguished element.

\footnotetext{
${ }^{2}$ We are essentially working with $b$ 's which are sets of ordinals, and sweeping some codings under the rug.
} 
Iterations of a relativized premouse $M$ are always by extenders on its coherent sequence, all of which have critical points above $\dot{b}^{M}$. A relativised premouse $M$ is countably iterable if whenever $\pi: N \rightarrow M$ is $\mathcal{L}_{0^{-}}$elementary with $N$ countable then $N$ is $\omega_{1}+1$ iterable. Fix $b$ transitive. Any two sound countably iterable premice over $b$ which project to $b$ are comparable (see [20]). (Their possibly different parameters are irrelevant at this point.) The lower part closure of $b$ is defined as the union of all $b$-premice $N$ which are countably iterable, sound, and satisfy $\rho_{\omega}(M)=b . L p(b)$ can be regarded as a countably iterable $b$-premouse in its own right, over any $a \in b$. We sometimes write $L p^{a}(b)$ when we want to think of it this way, but we shall drop the superscript $a$ when it is safe to do so.

Definition 14. Let $\nu \geq \omega_{1}$ be regular, and $a \in H(\nu)$. Let $\varphi$ be an $r Q$-sentence of $\mathcal{L}_{0}$. Suppose that for any transitive, selfwellordered $b \in H(\nu)$ such that $a \in b$, there is a countably iterable premouse $M$ over $b$ with parameter $a$ such that $M \models \varphi$; then we set

$$
J_{\varphi}(b)=L p^{a}(b) \mid \gamma
$$

where $\gamma$ is least such that $L p^{a}(b) \models \varphi$. We call the map $b \mapsto J_{\varphi}(b)$ a (first order) $(\nu, a)$ mouse operator. We say that $J_{\varphi}$ is defined on the $H(\nu)$-cone above $a$.

Remark 15. In some contexts, the countable iterability requirement we have imposed above is too onerous. For example, if $H\left(\omega_{1}\right)$ is closed under sharps, then $\omega_{1}$-iterability is enough to identify the true $M_{1}^{\sharp}$. We don't need full $\omega_{1}+1$ iterability. This fact will be important for us when we consider mice in $V[h]$, for $h$ generic over $\operatorname{Col}\left(\omega, \omega_{2}\right)$. Our hypotheses are consistent with $V=L[A]$ for some $A \subseteq \omega_{3}$, so they do not imply that $M_{1}^{\sharp}$ is $\omega_{1}+1$ iterable in $V[h]$. (An iteration to make $A$ generic will provide a counterexample.) We do have to consider countable mice in $V[h]$, in order to show the mouse operators in smaller models behave well. However, we don't need to consider mouse operators in $V[h]$, so we can stick with the $\omega_{1}+1$ iterability requirement of 14 .

An important property of first order mouse operators is that they condense well, in the sense of the following lemma.

Lemma 16. Let $J$ be a first order mouse operator with parameter a. Let $b \in \operatorname{dom}(J)$, and let $\pi: \mathcal{M} \rightarrow J(b)$ be $r Q$-elementary, with $\pi(c)=b$, and $\pi \uparrow T C(a \cup\{a\})$ being the identity; then $c \in \operatorname{dom}(J)$, and $\mathcal{M}=J(c)$.

One theme of this paper is that simultaneous reflection can be used to lift closure under certain operations from $P\left(\omega_{1}\right)$ to $P\left(\omega_{2}\right)$. In [25], 
Woodin gives a proof that under $\mathrm{WRP}_{(2)}\left(\omega_{2}\right)$, closure of $P\left(\omega_{1}\right)$ under sharps entails closure of $P\left(\omega_{2}\right)$ under sharps. His proof of Theorem 10 involved analogous arguments for the $M_{n}^{\#}$ operation. The following is a straighforward generalization to first order mouse operators. There is a related argument in [27], where it is shown that under $\operatorname{WRP}_{(2)}\left(\omega_{2}\right)$, $\omega_{1}$-Universally Baire self-justifying systems are $\omega_{2}$-Universally Baire. What is key to all the arguments is that the function being extended from $P\left(\omega_{1}\right)$ to $P\left(\omega_{2}\right)$ condenses well.

Definition 17. For regular cardinals $\kappa<\lambda$, we say that Mouse Reflection holds at $(\kappa, \lambda)$ iff for every $a \in H(\kappa)$, every $(\kappa, a)$-mouse operator can be extended to a $(\lambda, a)$-mouse operator. If $\lambda=\kappa^{+}$, we say that Mouse Reflection holds at $\kappa$.

Lemma 18. $\operatorname{WRP}_{(2)}\left(\omega_{2}\right)$ implies Mouse Reflection at $\omega_{2}$.

Proof. Let $J=J_{\varphi}$ be a first order mouse operator with parameter a defined on the $H\left(\omega_{2}\right)$ cone above $a$. Fix a transitive, selfwellordered $b$ in $H\left(\omega_{3}\right)$ such that $a \in b$. We must show that there is a countably iterable $b$-premouse with parameter $a$ that satisfies $\varphi$.

For $\sigma \in[b]^{\omega}$ such that $a \in \sigma$, let $b_{\sigma}$ be the transitive collapse of $\sigma$, and let $a_{\sigma}$ be the collapse of $a$.

Claim. For club many $\sigma \in[b]^{\omega}$, there is a countably iterable $b_{\sigma^{-}}$ premouse $\mathcal{M}$, with parameter $a_{\sigma}$, such that $\mathcal{M} \models \varphi$.

Proof. If not, then one-set stationary reflection for $[b]^{\omega}$ gives us an $X \subseteq b$ such that $|X|=\omega_{1}, T C(a \cup\{a\}) \subseteq X$, and for stationary many $\sigma \in[X]^{\omega}$ the conclusion of the claim fails. But let $b_{X}$ be the collapse of $X$, and note that $a$ is fixed by this collapse. Let $\mathcal{N}=J_{\varphi}\left(b_{X}\right)$. It is clear that for club many $\sigma \in[X]^{\omega}$, there is a countable $Y \prec \mathcal{N}$ such that $Y \cap X=\sigma$. For such $\sigma$, the collapse of $Y$ is an $\mathcal{M}$ as in the claim. Contradiction.

For $c \in \operatorname{dom}(J)$, let $T^{J}(c)=\left\{\langle\psi, t\rangle \mid t \in c^{<\omega} \wedge \psi \in \mathcal{L}_{0} \wedge J(c) \models \psi[t]\right\}$. Since $J(c)$ projects to $c$, it is coded by $T^{J}(c)$. Our goal is to define the appropriate theory to be $T^{J}(b)$. To this end, for $t \in[b]^{<\omega}$ and $\psi(v)$ an $\mathcal{L}_{0}$ formula, put

$$
S_{\psi, t}=\left\{\sigma \in[b]^{\omega} \mid t_{\sigma} \in T^{J}\left(b_{\sigma}\right)\right\} .
$$

Claim. For any $\psi, t$, one of $S_{\psi, t}$ and $S_{\neg \psi, t}$ contains a club in $[b]^{\omega}$.

Proof. Otherwise we can find $X \subseteq b$ such that $|X|=\omega_{1}, t \in X$, $T C(a \cup\{a\}) \subseteq X$, and both $S_{\psi, t}$ and $S_{\neg \psi, t}$ are stationary in $[X]^{\omega}$. Let $b_{X}$ be the transitive collapse of $X$, and $t_{X}$ the image of $t$ under the collapse. The collapse fixes $a$. Arguing as in the first claim, we 
see that if $J\left(b_{X}\right) \models \psi\left[t_{X}\right]$, then $S_{\psi, t}$ contains a club in $\left.b_{X}\right]^{\omega}$, and if $J\left(b_{X}\right) \models \neg \psi\left[t_{X}\right]$, then $S_{\neg \psi, t}$ contains a club in $\left[b_{X}\right]^{\omega}$. In either case, we have a contradiction.

Now we put

$$
\langle\psi, t\rangle \in T \Leftrightarrow S_{\psi, t} \text { contains a club in }[b]^{\omega} .
$$

It is easy to see that $T$ is the theory with parameters of a countably iterable $b$-premouse $\mathcal{M}$ with parameter $a$ satisfying $\varphi$.

Remark 19. We believe that with more work, one can show that simultaneous reflection for pairs of stationary subsets of $\omega_{2}$ implies Mouse Reflection at $\omega_{2}$.

Definition 20. Let $J$ be a $(\nu, a)$-mouse operator, and let $\mathcal{M}$ be a $b$ premouse with parameter $a$; then we say $\mathcal{M}$ is $J$-level-closed iff whenever $\eta<\xi<\nu$ and $\xi$ is a cardinal of $\mathcal{M}$, then $J(\mathcal{M} \mid \xi) \unlhd \mathcal{M}$.

For any $(\nu, a)$-mouse operator $J$ and set $b$ in its domain there is a corresponding minimal $J$-level-closed premouse $L^{J}(b)$, obtained by concatenating extender sequences. A condensation argument shows that $L^{J}(b)$ is a bone-fide $b$-premouse with parameter $a$. It has ordinal height $o\left(L^{J}(b)\right)=\nu$, and it is countably iterable.

If they are defined on the $H(\nu)$ cone above $a$, then $J^{\sharp}, J^{*}$, and $J_{n}^{w}$ are themselves $(\nu, a)$-mouse operators. The reader should see [22] and [16] for background.

Definition 21. A $(\nu, a)$-mouse operator $J$ relativises well iff

(1) there is a formula $\theta(u, v, w, z)$ such that whenever $b, c \in \operatorname{dom}(J), b \in$ $c$, and $N$ is a transitive model of $\mathrm{ZFC}^{-}$such that $J(c) \in N$, then $J(b) \in N$ and $J(b)$ is the unique $x \in N$ such that $N \models$ $\theta[x, a, b, J(c)]$, and

(2) if $b \in \operatorname{dom}(J)$ and $\eta$ is a cutpoint of $J(b)$, then $J(J(b) \mid \eta)$ is not a proper initial segment of $J(b)$.

We shall only be dealing with operators that relativize well. Clause (2) is used in the proof of

Lemma 22. Suppose that $J$ is a $(\nu, a)$ mouse operator that relativises well; then for all $b \in \operatorname{dom}(J), J(b)$ is $\nu$-iterable.

Proof. We show that $J(b)$ is iterable by the strategy of choosing the unique cofinal branch $b$ of $\mathcal{T}$ such that $Q(b, \mathcal{T}) \unlhd J(\mathcal{M}(\mathcal{T}))$. The usual reflection argument shows that this works. 
If $J$ relativizes well, then a $J$-level-closed premouse that satisfies ZFC $^{-}$is fully closed under $J$. Because of this, we shall sometimes say "J-closed" when we mean "J-level-closed". Note that if $n \leq \omega$ and the $M_{n}^{\sharp}$ operator is total on $H(\nu)$, then it is a $(\nu, 0)$-mouse operator that relativizes well. Another very useful property of the $M_{n}^{\sharp}$ operator is that it determines itself on generic extensions, in the following sense.

Definition 23. Let $J$ be a $(\nu, a)$-mouse operator; we say that $J$ determines itself on generic extensions iff for all $b$ in the $H\left(\omega_{1}\right)$-cone over $a$, and all $g$ that are $\mathbb{P}$-generic over $J(b)$ for some $\mathbb{P} \in \operatorname{rud}(b)$, we have that $J(b)[g]=J(\langle b, g\rangle)$.

Notice here that $J(b)[g]$ can be regarded as a premouse over $\langle b, g\rangle$, because the forcing is small with respect to extenders on the sequence of $J(b)$. Definition 23 requires that so regarded, $J(b)[g]$ is just $J(\langle b, g\rangle$. It is clear that if the $M_{n}^{\sharp}$ operator is defined on $H\left(\omega_{1}\right)$, then it determines itself on generic extensions.

It is shown in [21][section 4] that under $A D$, every mouse operator on $H\left(\omega_{1}\right)$ determines itself on generic extensions in some $H\left(\omega_{1}\right)$ cone.

Although we stated definition 23 in terms of generic extensions of countable models that exist in $V$, condensation leads to extendibilty beyond $V$ :

Lemma 24. Let $a \in H\left(\omega_{1}\right)$, and let $J=J_{\varphi}$ be a first order $(\nu, a)$ mouse operator, where $\nu \geq \omega_{1}$ is regular. Suppose that $J \uparrow H\left(\omega_{1}\right)$ relativizes well and determines itself on generic extensions. Let $h$ be $V$-generic over some partial order of size $<\nu$; then $V[h]$ satisfies "for any c in the $H(\nu)$ cone over a, there is a c-premouse $\mathcal{M}$ with parameter a such that $\mathcal{M}=\varphi$, and $\mathcal{M}$ is $\nu$-iterable.

Proof. Let $\theta$ be the formula witnessing that $J$ relativises well. Let $\tau \in H(\nu)$ be a term such that $c=\tau_{h}$. Let $b$ be in the $H(\nu)^{V}$ cone over $a$ with $\tau \in b$.

The canonical re-arrangement of $J(b)[h]$ is a $\langle b, h\rangle$-premouse with parameter $a$ satisfying $\varphi$. For if not, we can find in $V$ a countable elementary submodel $N$ of $V$ such that forcing over $N$ yields $g$, where $\bar{J}(\bar{b})[g]$ does not re-arrange to a premouse satisfying $\varphi$. But $\bar{J} \subseteq J \uparrow$ $H\left(\omega_{1}\right)$ because $J$ condenses well. Since $J$ determines itself on generic extensions, we have a contradiction.

Let $\mathcal{N}$ be the canonical re-arrangement of $J(b)[h]$. Let $\theta$ be the formula witnessing that $J \uparrow H\left(\omega_{1}\right)$ relativises well. let $M \models \mathrm{ZFC}^{-}$ be transitive, with $b, h \in M$. A Lowenheim-Skolem argument like that in the last paragraph shows that there is a unique $c$-premouse $\mathcal{M}$ 
satisfying $\varphi$ such that for some (equivalently all) transitive $S \models$ ZFC $^{-}$, $S \models \theta[\mathcal{M}, a, c, \mathcal{N}]$. This is our desired $\mathcal{M}$.

If $\nu=\omega_{1}$ in $V[h]$, the premouse $\mathcal{M}$ in the conclusion of 24 may not be $\omega_{1}+1$-iterable in $V[h]$. See remark 15 above. It is, however, definable in $V[h]$ from $J \uparrow V$ and $c$, uniformly over all $V[h]$. Thus we write

$$
\mathcal{M}=J^{h}(c)
$$

for the $c$-premouse satisfying $\varphi$ and obtained from $J$ as above. If $\nu>\omega_{1}$ in $V[h]$, we get

Corollary 25. Under the hypotheses of 24, if $\nu>\omega_{1}$ in $V[h]$, then $J^{h}$ is a $(\nu, a)$ mouse operator extending $J$, and $J^{h}$ relativizes well, and determines itself on generic extensions.

Definition 26. Let $I$ be a presaturated ideal on $\omega_{1}$, and suppose $2^{\omega_{1}}=\omega_{2}$. Let $a \in H\left(\omega_{1}\right)$, and let $J$ be a $\left(\omega_{3}, a\right)$ mouse operator that relativizes well and determines itself on generic extensions. We say that $J$ is $I$-absolute iff whenever $\pi: V \rightarrow \operatorname{Ult}(V, G)$ is a generic embedding associated to some $\left(I^{+}, \subseteq\right.$ )-generic $G$, and $J^{G}$ is the extension of $J$ to $V[G]$ given by corollary 25 , then

$$
\pi(J) \uparrow H\left(\omega_{1}\right)^{V[G]}=J^{G} \uparrow H\left(\omega_{1}\right)^{V[G]} .
$$

If $J$ is $I$-absolute, then in fact $\pi(J) \subseteq J^{G}$ in full, by a simple Lowenheim-Skolem argument based on the condensation property of $J$.

Definition 27. Let $J$ be a $(\nu, a)$-mouse operator, and let $b \in \operatorname{dom}(J)$; then

(1) $J^{\sharp}(b)$ is the minimal active, countably iterable, $J$-level-closed $b$-premouse, if there is one.

(2) $J^{*}(b)=\left(J^{\sharp}\right)^{\sharp}(b)$, if it exists.

(3) For $n \geq 1, J_{n}^{w}(b)=M_{n}^{J, \sharp}(b)$ is the minimal active, countably iterable, $J$-level-closed $b$-premouse satisfying "there are $n$ Woodin cardinals above $o(b) "$, if there is one. We put $J^{w}(b)=J_{1}^{w}(b)$.

If they are defined on the $H(\nu)$ cone above $a$, then $J^{\sharp}, J^{*}$, and $J_{n}^{w}$ are themselves $(\nu, a)$-mouse operators. The reader should see [22] and [16] for background.

The successor step in a core model induction is the step from $J$ closure to $J^{w}$-closure. We are ready now to execute it, in the case that our given $J$ is a first order mouse operator.

Theorem 28. Suppose that I s a presaturated ideal on $\omega_{1}, 2^{\omega_{1}}=\omega_{2}$, and Mouse Reflection holds at $\omega_{2}$. Let $a \in H\left(\omega_{1}\right)$, and suppose that $J$ 
is a first order $\left(\omega_{3}, a\right)$-mouse operator that relativizes well, determines itself on generic extensions, and is I-absolute; then $J^{w}$ is a first order $\left(\omega_{3}, a\right)$-mouse operator that relativizes well, determines itself on generic extensions, and is I-absolute.

Proof. We first take a smaller step.

Claim 1. $J^{\sharp}$ is an $\left(\omega_{3}, a\right)$ mouse operator that relativizes well, determines itself on generic extensions, and is $I$-absolute.

Proof. Let $G$ be $V$-generic over $\left(I^{+}, \subseteq\right)$, and

$$
\pi: V \rightarrow M=\operatorname{Ult}(V, G)
$$

be the generic embedding. We have that $M$ is closed under $\omega$-sequences in $V[G], \pi\left(\omega_{1}^{V}\right)=\omega_{2}^{V}, \pi\left(\omega_{3}^{V}\right)=\omega_{3}^{V}=\omega_{2}^{V[G]}$, and

$$
V[G] \models\{\alpha \mid \pi(\alpha)=\alpha\} \text { is stationary in } \omega_{2} .
$$

By corollary 25, there is in $V[G]$ a unique $\left(\omega_{2}^{V[G]}, a\right)$-mouse operator $J^{G}$ extending $J$. Because $J$ is $I$-absolute, $V[G] \models \pi(J) \uparrow H\left(\omega_{1}\right)=$ $J^{G} \uparrow H\left(\omega_{1}\right)$. It follows from this that

$$
\pi(J) \subseteq J^{G} .
$$

For take any $c \in \operatorname{dom}(\pi(J))$; then $c \in \pi\left(H\left(\omega_{3}\right)\right) \subseteq H\left(\omega_{2}\right)^{V[G]}$, so $c \in$ $\operatorname{dom}\left(J^{G}\right)$. If $J^{G}(c) \neq \pi(J)(c)$, then by a simple Skolem hull argument in $V[G]$, using condensation for $J^{G}$ in $V[G]$ and for $\pi(J)$ in $M$, we get a countable $b$ such that $J^{G}(b) \neq \pi(J)(b)$. This is a contradiction.

Now let $b$ be in the $H\left(\omega_{1}\right)$-cone over $a$ of $V$. We wish to show that in $V, J(b)^{\sharp}$ exists and is countably iterable. This is basically a well known result of Kunen, but we sketch a proof for completeness. Setting $\theta=\omega_{3}^{V}$, we have in $V[G]$

$$
\pi: L_{\theta}^{J}(b) \rightarrow L_{\theta}^{J}(b)
$$

with a stationary set of fixed points. We leave it to the reader to show that $\omega_{1}^{V}$ and $\omega_{2}^{V}$ are inaccessible cardinals in $L^{J}(b)$. Let $\kappa_{0}=\omega_{1}^{V}$, and let

$$
U_{0}=\left\{A \subseteq \kappa_{0} \mid A \in L^{J}(b) \wedge \kappa_{0} \in \pi(A)\right\} .
$$

Let $\mu=\left(\kappa_{0}^{+}\right)^{L^{J}(b)}$, and

$$
\mathcal{M}_{0}=\left(L_{\mu}^{J}(b), \in, U_{0}\right) .
$$


$\mathcal{M}_{0}$ is an amenable structure by an argument of Kunen. Let $\mathcal{M}_{\alpha}$ be the $\alpha$-th iterate of $\mathcal{M}_{0}$ by $U_{0}$ and its images. We show by induction on $\alpha<\omega_{1}^{V[G]}$ that $\mathcal{M}_{\alpha}$ has the form $\left(L_{\mu_{\alpha}}^{J}(b), \in, U_{\alpha}\right)$, where $\mu_{\alpha}$ is a cardinal of $L_{\theta}^{J}(b)$. At the same time we define maps $i_{\beta, \alpha}: L_{\theta}^{J}(b) \rightarrow L_{\theta}^{J}(b)$ extending the iteration map from $\mathcal{M}_{\beta}$ to $\mathcal{M}_{\alpha}$, and "realization maps"

$$
\pi_{\alpha}: L_{\theta}^{J}(b) \rightarrow L_{\theta}^{J}(b),
$$

with $\pi_{0}=\pi$, such that for all $\beta<\alpha$

$$
\pi_{\beta}=\pi_{\alpha} \circ i_{\beta, \alpha}
$$

If $\alpha$ is a limit ordinal, then $i_{\beta, \alpha}$ is the direct limit map, and $\pi_{\alpha}\left(i_{\beta, \alpha}(x)\right)=$ $\pi_{\beta}(x)$ for all $x$. Note that $\pi_{\alpha}$ embeds the direct limit into $L_{\theta}^{J}(b)$, and thus the direct limit does indeed have the form $L_{\theta}^{J}(b)$, by the fact that $J$ condenses well. We let

$$
i_{\alpha, \alpha+1}: L_{\theta}^{J}(b) \rightarrow \operatorname{Ult}\left(L_{\theta}^{J}(b), U_{\alpha}\right)
$$

be the ultrapower map, and

$$
\pi_{\alpha+1}\left(i_{\alpha, \alpha+1}(f)\left(\kappa_{\alpha}\right)\right)=\pi_{\alpha}(f)\left(\kappa_{\alpha}\right)
$$

where $\kappa_{\alpha}=\operatorname{crit}\left(U_{\alpha}\right)=i_{0, \alpha}\left(\kappa_{0}\right)$. This works so long as $U_{\alpha}$ is the ultrafilter derived from $\pi_{\alpha}$, that is, $\operatorname{crit}\left(\pi_{\alpha}=\kappa_{\alpha}\right.$, and for $X \subseteq \kappa_{\alpha}$ in $L_{\theta}^{J}(b)$,

$$
(*) X \in U_{\alpha} \Leftrightarrow \kappa_{\alpha} \in \pi_{\alpha}(X) .
$$

Again, the fact that $J$ condenses well then yields that $\operatorname{Ult}\left(L_{\theta}^{J}(b), U_{\alpha}\right)=$ $L_{\theta}^{J}(b)$, as desired.

We omit the proof that $\operatorname{crit}\left(\pi_{\alpha}\right)=\kappa_{\alpha}$. To see $(*)$, let $\nu<\mu_{0}$, $W=U_{0} \cap L_{\nu}^{J}(b)$, and $f: \kappa_{0} \rightarrow P\left(\kappa_{0}\right) \cap L_{\nu}^{J}(b)$. Let $c, \tau$ be such that $\pi(c)=c$, and for all $\xi<\kappa_{0}, f(\xi)=\tau^{L_{\theta}^{J}(b)}[c](\xi) \cap \kappa_{0}$. Then

$$
L_{\theta}^{J}(b) \models \forall \xi<\kappa_{0}\left(\left(\tau[c](\xi) \cap \kappa_{0} \in W\right) \leftrightarrow \kappa_{0} \in \tau[c](\xi)\right) .
$$

This fact is preserved by $i_{0, \alpha}$, and from that, we easily get $(*)$.

So for $b$ in the $H\left(\omega_{1}\right)$ cone over $a$ of $V, V[G] \models J^{\sharp}(b)$ exists, and is $\omega_{2}$ iterable. (The iterations that do not drop are linear, so we can go to $\omega_{2}^{V[G]}$. This uses 24 for iterations that do drop.) Let $\mathcal{M}=J^{\sharp}(b)^{V[G]}$, and let $h$ be $V$-generic over $\operatorname{Col}\left(\omega, \omega_{2}\right)$ and such that $G \in V[h]$. Our proof shows that $\mathcal{M}$ is definable in $V[h]$ from $b$ and $J^{V}$ : it is the unique putative $J(b)^{\sharp}$ that is linearly $\omega_{1}$-iterable by its last extender in a way that moves the $L^{J}(b)$ to itself. It follows that $\mathcal{M} \in V$, and it is easy to see that it is countably iterable in $V$. So for $b$ in the $H\left(\omega_{1}\right)$ cone over $a$ of $V, V \models J^{\sharp}(b)$ exists. 
Now let $b \in H\left(\omega_{2}\right)^{V}$. Since $b \in H\left(\omega_{1}\right)^{M}$, we have an $\mathcal{N}$ such that $M \models \mathcal{N}=\pi(J)^{\sharp}(b)$. Since $J$ is $I$-absolute, this gives

$$
V[G] \models \mathcal{N}=\left(J^{G}\right)^{\sharp}(b),
$$

with $\mathcal{N}$ being $\omega_{2}$-iterable in $V[G]$. As in the last paragraph, this gives that $\mathcal{N}$ is definable from $J^{V}$ and $b$ in $V^{\operatorname{Col}\left(\omega, \omega_{2}\right.}$, so $\mathcal{N} \in V$, and $V \models$ $\mathcal{N}=J^{\sharp}(b)$.

Finally, $J^{\sharp}$ can be extended to $H\left(\omega_{3}\right)^{V}$ by Mouse Reflection at $\omega_{2}$.

It is easy to see that $J^{\sharp}$ relativizes well, determines itself on generic extensions, and is $I$-absolute. Indeed, the proof that it is $I$-absolute is part of our proof that that $J^{\sharp}$ is defined on $H\left(\omega_{2}\right)^{V}$.

Claim 2. $J^{*}$ is an $\left(\omega_{3}, a\right)$ mouse operator that relativizes well, determines itself on generic extensions, and is $I$-absolute.

Proof. $\quad J^{*}=\left(J^{\sharp}\right)^{\sharp}$, so we can just use the proof of claim 1, with $J^{\sharp}$ replacing $J$.

We are ready to prove that $J^{w}$ an $\left(\omega_{3}, a\right)$ mouse operator that relativizes well, determines itself on generic extensions, and is $I$-absolute. We show first that it is an $\left(\omega_{1}, a\right)$ mouse operator. The proof is parallel to that in the step from $J$ to $J^{\sharp}$, but now the core model theory is too involved to be reproduced, so we must just quote it.

Let $b$ be in the $H\left(\omega_{1}\right)$ cone over $a$. Let $C \subseteq \omega_{2}$ code $\left\langle H\left(\omega_{2}\right), I\right\rangle$. We work in the model

$$
N=L_{\omega_{2}}^{J^{\sharp}}[C] .
$$

Because $J^{*}(C)$ exists, $N \models$ ZFC, and letting $\Omega$ be the critical point of the last extender of $J^{*}(C), \Omega$ behaves in $N$ enough like a measurable cardinal that the core model theory of [18], relativised to $J$, goes through. Let $K^{c, J}(b)$ be the result of the $J$-relativized $K^{c}$-construction over $b$ of length $\Omega$. (See $\left[16 .^{3}\right.$ ) It is enough to show that $K^{c, J}(b)$ reaches an active level $\mathcal{P}$ satisfying "there is a Woodin cardinal". That is because the first such $\mathcal{P}$ is countably iterable in $N$, and hence countably $J$-iterable ${ }^{4}$ in $V$ because $H\left(\omega_{2}\right) \subseteq N$. It follows that $\mathcal{P}$ can be re-arranged as a countably iterable putative $M_{1}^{J, \sharp}(b)$.

\footnotetext{
${ }^{3} J$ is fed into the model being constructed as a model operator, meaning that if the current model in the construction is $\mathcal{P}$, and we are not adding an extender, then the next model is the core of $\mathcal{Q}$, where $\mathcal{Q}=J(\mathcal{P})$ unless some proper initial segment of $J(\mathcal{P})$ projects strictly across $o(\mathcal{P})$, in which case $\mathcal{Q}$ is the first such initial segment of $J(\mathcal{P})$. Strictly speaking, the levels of $K^{c, J}(b)$ are not $b$-premice, but hybrid $b$-premice relative to the model operator $F^{J}$, where $F^{J}(\mathcal{P})$ is the $\mathcal{Q}$ we just described. See [16].

${ }^{4}$ This means that the iterations move $F^{J}$ correctly. See [16].
} 
But if the $K^{c, J}(b)$ construction does not reach such a $\mathcal{P}$, then in $N$, $K^{J}(b)$ exists, and has the basic properties of the unrelativized $K$ from [18]. Since $I$ is a presaturated ideal in $N$, the argument of [18] [section 7] leads to a contradiction. This shows that $J^{w}$ is an $\left(\omega_{1}, a\right)$ mouse operator. It is easy to see that it relativizes well and determines itself on generic extensions, using those properties of $J$.

We now extend $J^{w}$ to an $I$-absolute $\left(\omega_{2}, a\right)$ operator. Let $b$ be in the $H\left(\omega_{2}\right)$ cone over $a$. Let

$$
\pi: V \rightarrow M=\operatorname{Ult}(V, G)
$$

be the generic embedding associated to $G \subseteq I^{+}$. Let $\mathcal{P}$ be such that

$$
M \models \mathcal{P}=M_{1}^{\pi(J), \sharp}(b) .
$$

Claim. $\mathcal{P}$ is in $V$, and countably iterable in $V$.

Proof. Let $h$ be $V$-generic over $\operatorname{Col}\left(\omega, \omega_{2}\right)$, and such that $G \in V[h]$. In $V[h], \mathcal{P}$ is $\omega_{1}$-iterable by the following strategy $\Sigma$ : pick the unique cofinal branch $b$ of $\mathcal{T}$ such that $\mathcal{Q}(b, \mathcal{T}) \unlhd\left(J^{\sharp}\right)^{h}(\mathcal{M}(\mathcal{T}))$. To see that $\Sigma$ works, note that it works in $V[G]$, because $M$ and $V[G]$ have the same reals, and $\pi(J) \subseteq J^{G}$. But $H\left(\omega_{2}\right)^{V[G]}[h]=H\left(\omega_{1}\right)^{V[h]}$, and $\left(J^{\sharp}\right)^{G}$ determines $\left(J^{\sharp}\right)^{h}$ on $H\left(\omega_{1}\right)^{V[h]}$. A simple Lowenheim Skolem argument for the forcing from $V[G]$ to $V[h]$ shows that if $\Sigma$ fails in $V[h]$, it fails in $V[G]$. Thus $\Sigma$ works in $V[h]$. Note that $\Sigma$ is definable from $J^{\sharp}$, which is in $V$.

If $\mathcal{Q}, \Lambda$ have in $V[h]$ the properties of $\mathcal{P}, \Sigma$ just described, then working in $J^{*}(\langle\mathcal{P}, \mathcal{Q}\rangle)$, where both $\mathcal{P}$ and $\mathcal{Q}$ are $\omega_{1}+1$-iterable, we can compare them. This shows $\mathcal{Q}=\mathcal{P}$. It follows that $\mathcal{P}$ is definable in $V[h]$ from $b$ and $\left(J^{*}\right)^{V}$, and thus $\mathcal{P} \in V$ by the homogeneity of $\operatorname{Col}\left(\omega, \omega_{2}\right)$. For a similar reason, $\Sigma \uparrow V \in V$, and witnesses that $\mathcal{P}$ is countably iterable (in fact $\omega_{3}$-iterable) in $V$.

Thus $J^{w}$ extends to an $\left(\omega_{2}, a\right)$ mouse operator, and our proof showed that it is $I$-absolute. By Mouse Reflection at $\omega_{2}, J^{w}$ extends to an $\left(\omega_{3}, a\right)$ mouse operator. The extension relativises well and determines itself on generic extensions, because these properties depend only on $J \uparrow H\left(\omega_{1}\right.$. It is $I$-absolute, because this property depends only on $J \uparrow H\left(\omega_{2}\right)$.

This completes the proof of theorem 28.

We have at once 
Corollary 29. Suppose there is a presaturated ideal on $\omega_{1}$, and $2^{\omega_{1}}=$ $\omega_{2}$, and Mouse Reflection holds at $\omega_{2}$; then for all $n<\omega$ and all $b \in H\left(\omega_{3}\right), M_{n}^{\sharp}(b)$ exists, and is countably iterable. Thus PD holds, and continues to hold in $V[H]$ whenever $H$ is $V$-generic for a poset of size $\leq \omega_{2}$.

\section{An ASIDE ON $2^{\omega_{1}}=\omega_{2}$}

We digress briefly in order to prove the following theorem, due to Woodin and implicit in [25] (see Thm. 9.82 for example).

Lemma 30. Assume $\mathrm{WRP}_{(2)}\left(\omega_{2}\right)$ and NS saturated; then

$$
2^{\omega}=2^{\omega_{1}}=\delta_{2}^{1}=\omega_{2} .
$$

Proof. A theorem of Todorcevic (see Thm 6.4 of [6]) gives $2^{\omega} \leq \omega_{2}$ under $\operatorname{WRP}\left(\omega_{2}\right)$. The idea is that there is always an injection from $2^{\omega}$ into any club subset $C$ of $\left[\omega_{2}\right]^{\omega}$, and under $\operatorname{WRP}\left(\omega_{2}\right)$ there is such a club of size $\omega_{2}$, namely

$$
C=\bigcup_{\delta<\omega_{2}} C_{\delta}
$$

where each $C_{\delta}$ be a club of size $\omega_{1}$ in $[\delta]^{\omega}$. Now, NS saturated gives closure of $P\left(\omega_{1}\right)$ under the dagger operation and $\operatorname{WRP}_{(2)}\left(\omega_{2}\right)$ lifts this closure to $P\left(\omega_{2}\right)$ as in Lemma 18 below. For $B \subseteq \omega_{2}$, we write $L[B, U]$ for the minimal model with one measurable cardinal over $B$ described by $B^{\dagger}$. We construct a function $B: \omega_{2} \rightarrow P\left(\omega_{1}\right)$ as follows. Let $B(0) \subset$ $\omega_{1}$ be such that $\omega_{1}^{L[B]}=\omega_{1}$. Given $B \uparrow \gamma$, let $\left(X_{\xi} \mid \xi<\omega_{1}\right)$ enumerate those nonstationary sets $X \in L[B \uparrow \gamma, U]$ which are stationary in $L\left[B\lceil\gamma, U]\right.$. Let $B(\gamma)$ code a sequence of clubs disjoint from the $X_{\xi}$ as well as a surjection from $\omega_{1}$ to $\gamma$. Every subset of $\omega_{2}$ in the final $L[B, U]$ is in $L[B \uparrow \gamma, U]$ for some $\gamma<\omega_{2}$, so $L[B, U]$ thinks that $N S$ is saturated, and computes $\omega_{2}$ correctly. Since $L[B, U]$ has a measurable cardinal, it follows from 3.17 of [25] that $\delta_{2}^{1}=\omega_{2}$ in $L[B, U]$, and thus $2^{\omega}=\omega_{2}$ in $V$, and we are left to show that $2^{\omega_{1}} \leq \omega_{2}$.

For this, let $G$ be $V$-generic over $(N S, \subseteq)$, and let $j: V \rightarrow M \simeq$ $\operatorname{Ult}(V, G)$ be the generic embedding. We have $\omega_{2}^{V}=\omega_{1}^{V[G]}$ and $\omega_{3}^{V}=$ $\omega_{2}^{V[G]}$ by the $\omega_{2}$-c.c. Thus

$$
j\left(\omega_{2}^{V}\right)=\omega_{2}^{M} \leq \omega_{2}^{V[G]}=\omega_{3}^{V} .
$$

But $j$ is continuous at $\omega_{2}$, so

$$
j\left(\omega_{2}^{V}\right)<\omega_{3}^{V} .
$$

But $M=2^{\omega}=\omega_{2}$, and $M$ contains all the reals of $V[G]$, so $\mathrm{CH}$ holds in $V[G]$. On the other hand, if $V$ thinks that $f$ is an injection of $\omega_{3}$ 
into $P\left(\omega_{1}\right)$, then $V[G]$ thinks that $f$ enumerates $\omega_{2}$ distinct subsets of $\omega_{1}^{V}$, contrary to $\mathrm{CH}$.

The hypothesis that $2^{\omega} \leq \omega_{2}$ is not needed for corollary 29, and probably not for theorem 28 , though we have not checked the latter carefully. The following little lemma lets us drop the hypothesis.

Lemma 31. Suppose $I$ is a presaturated ideal on $\omega_{1}$ and $J$ is an $\left(\omega_{3}, a\right)$ mouse operator, where $a \in H\left(\omega_{2}\right)$. Suppose that $J^{\sharp}$ is an $\left(\omega_{2}, a\right)$-mouse operator; then there is a $B \subset \omega_{2}$ such that

(1) $H\left(\omega_{2}\right)^{L^{J}(B)}$ is fully elementary in $H\left(\omega_{2}\right)$

(2) $\bar{I}=I \cap L^{J}(B) \in L^{J}(B)$ and

(3) $L^{J}(B) \models \bar{I}$ is a presaturated ideal on $\omega_{1}$.

Proof. We inductively fold in all of the necessary data into $B$, which we regard as a function from $\omega_{2}$ to $P\left(\omega_{1}\right)$. Let $B(0)$ code $a$, and be such that $\omega_{1}^{L[B(0)]}=\omega_{1}$. Let $\delta<\omega_{2}$, and suppose $B \uparrow \delta$ has been defined. We let $B(\delta)$ code $W_{\delta}$ and $I_{\delta}$, where $I_{\delta}$ in turn codes

$$
I \cap J^{\#}(B \uparrow \delta)
$$

and $W_{\delta} \in\left[P\left(\omega_{1}\right)\right]^{\omega_{1}}$ has the following property: if $c \in \mathcal{M}^{\#}(B \uparrow \delta)$ and $\phi(x, y)$ is a formula of the language of set theory so that

$$
H\left(\omega_{2}\right)^{V} \models \phi[c, d]
$$

for some $d$, then there is such a $d$ coded (in some simple way) by an element of $W_{\delta}$.

Finally, we let

$$
\bar{I}=\bigcup \delta<\omega_{2} I_{\delta}
$$

It is easy to check (1)-(3) of the lemma.

\section{5. $I_{\alpha}$ AND COHEN FORCING}

In this section, we discuss our resemblance hypothesis $I_{\alpha}$, and we prove some lemmas on Cohen forcing over models of AD that are relevant to its formulation.

5.1. The role of $I_{\alpha}$. In section 3, we proved PD by a "cycling" argument, first closing $H\left(\omega_{1}\right)$ under a mouse operator, then lifting this closure to $H\left(\omega_{2}\right)$ and finally to $H\left(\omega_{3}\right)$. One idea of [26] is that the main obstacle to continuing this "cycling" argument through the levels of $L(\mathbb{R})$ is the lack of homogeneity of the forcing $P\left(\omega_{1}\right) / N S$. In order to make the $H\left(\omega_{1}\right)$ to $H\left(\omega_{2}\right)$ step, we need at least enough homogeneity that for $J$ the current mouse operator on $H\left(\omega_{1}\right)^{V}, \pi(J) \uparrow H\left(\omega_{2}\right)^{V}$ is independent of the NS-generic embedding $\pi$. J will be definable 
at some level of $L(\mathbb{R})^{V}$, and $\pi^{G}(J)$ at some level of $L(\mathbb{R})^{V[G]}$. This makes it natural to relate the $L(\mathbb{R})$ of the homogeneous extension by $\operatorname{Col}\left(\omega, \omega_{1}\right)$ that of an extension by $P\left(\omega_{1}\right) / N S \times \operatorname{Col}\left(\omega, \omega_{1}\right)$. This latter model $V^{P\left(\omega_{1}\right) / N S \times C o l\left(\omega, \omega_{1}\right)}$ is a Cohen extension of $V^{P\left(\omega_{1}\right) / N S}$, and a $c c c$ extension of $V^{C o l}\left(\omega, \omega_{1}\right)$ if $N S$ is saturated. The author of [26] believed that the existence of an embedding between the $L(\mathbb{R})$ of these models together with our hypotheses would yield $A D^{L(\mathbb{R})}$. That is, he conjectured the following weakening of our main theorem:

Assume $W R P_{(2)}\left(\omega_{2}\right)$ and NS saturated. Suppose that whenever $G \subset P\left(\omega_{1}\right) / N S$ is $V$-generic and $g \subset \operatorname{Col}\left(\omega, \omega_{1}\right)$ is $V[G]$-generic there is an embedding

$$
\bar{\pi}: L(\mathbb{R})^{V[g]} \rightarrow L(\mathbb{R})^{V[G][g]}
$$

which is $\Sigma_{1}$ and fixes ordinals. Then $A D$ holds in $L(\mathbb{R})$.

On the other hand, it was also known that if the induction were to succeed in proving $A D$ in $L(\mathbb{R})$, and that this persists after collapsing $\omega_{2}$, then we would have such an embedding. This follows easily from results of Foreman and Magidor ([3]) and Woodin ([2],[25]) that we shall use later in a similar way to prove $I_{\alpha}$. We therefore give the argument here. Let us first recall the Foreman-Magidor results.

Definition 32. ([3]) A partial ordering $\mathbb{P}$ is reasonable iff for all ordinals $\alpha,[\alpha]^{\omega} \cap V$ is stationary in $V^{\mathbb{P}}$.

All proper posets are reasonable, and thus all ccc posets are reasonable.

Definition 33. ([2]) A set $B$ of reals is $\kappa$-universally Baire just in case there are trees $T$ and $U$ on some $\omega \times \gamma$ such that $p[T]=B$, and whenever $\mathbb{P}$ has cardinality $<\kappa$, then $V^{\mathbb{P}} \models p[T]=\mathbb{R} \backslash p[U]$. We call $T$ and $U \kappa$-absolute complements in this situation.

Of course, we can speak of $\kappa$-universally Baire relations on reals as well. If $B$ is $\kappa$ universally Baire, and $T, U$ are $\kappa$-absolute complements such that $p[T]=B$, and $G$ is $V$-generic for a poset of size $<\kappa$, then we write $B^{G}$ for $p[T] \cap V[G]$. The notation is justified because if $T^{*}, U^{*}$ is another such complementing pair, then $p[T]=p\left[T^{*}\right]$ holds in $V[G]$. Foreman and Magidor note that if $B$ is an equivalence relation on $\mathbb{R}$, then $B^{G}$ is an equivalence relation on $\mathbb{R}^{V[G]}([3][3.3])$. It is also easy to see that if $B$ is a prewellorder of $\mathbb{R}$, then $B^{G}$ is a prewellorder of $\mathbb{R}^{V[G]}$. Foreman and Magidor show

Theorem 34. ([3][3.4]) Let $B$ be a $\kappa$-universally Baire prewellorder of $\mathbb{R}$, and let $G$ be $V$ generic over a reasonable poset of size $<\kappa$; then for every $x \in \mathbb{R}^{V[G]}$ there is a $y \in \mathbb{R}^{V}$ such that $\left.B^{G}(x, y)\right)$ and $B^{G}(y, x)$. 
That is, every $B^{G}$-equivalence class has a representative in $V$. In fact, [3][3.4] shows that if $B$ is a thin equivalence relation, then every $B^{G}$ equivalence class has a representative in $B$. One can show that the equivalence relation generated by a prewellorder with the Baire property is thin.

Theorem 35. Assume $N S$ saturated and $\mathrm{WRP}_{(2)}\left(\omega_{2}\right)$. Assume $\mathrm{AD}^{L(\mathbb{R})}$ holds in $V[g]$ whenever $g \subset \operatorname{Col}\left(\omega, \omega_{1}\right)$ is $V$-generic. Suppose $G$ and $g$ are the factors of generic filter on $P\left(\omega_{1}\right) / N S \times \operatorname{Col}\left(\omega, \omega_{1}\right)$. Then there is a fully elementary embedding

$$
\pi: L(\mathbb{R})^{V[g]} \rightarrow L(\mathbb{R})^{V[G][g]},
$$

and any such embedding satisfies $\pi \uparrow \theta^{L(\mathbb{R})}=i d$. Moreover, $A D^{L(\mathbb{R})}$ holds in $V$ and in the universe after collapsing $\omega_{2}$.

Proof. By Lemma 30, we have $2^{\omega_{1}}=\omega_{2}$. Thus from the perspective of $V[g]$ the algebra $\mathbb{B}=\left(P\left(\omega_{1}\right) / N S\right)^{V}$ has size $\omega_{1}$.

Claim 1. In $V[g] \models\left(P\left(\omega_{1}\right) / N S\right)^{V}$ has the countable chain condition.

Proof. Otherwise there is a condition $p \in \operatorname{Col}\left(\omega, \omega_{1}\right)$ which forces that some $\dot{f}$ enumerates an antichain of length $\omega_{2}^{V}$. On cardinality grounds there must be a condition $q \leq p$ which decides $\omega_{2}$ of the values of $\dot{f}$, a contradiction as two of these values must therefore be compatible by saturation of $N S$ in $V$.

Now, since $2^{\omega_{1}}=\omega_{2}$ and $P\left(\omega_{2}\right)$ is closed under sharps, we have $\mathbb{R}^{\#}$ in $V[g]$. By 9.83 of $[25]$ then we have that $\mathbb{R}^{\#}$ exists and $L(\mathbb{R}) \models A D$ in the universe after collapsing $\omega_{2}$. This uses $\operatorname{WRP}_{(2)}\left(\omega_{2}\right)$. Now we may assume there is $h \subset \operatorname{Col}\left(\omega, \omega_{2}\right)$ which is $V[G][g]$ generic and so that $V[G][g][h]=V[\bar{h}]$ for some $\bar{h} \subset \operatorname{Col}\left(\omega, \omega_{2}\right)$. The argument of 5.2 of [2] now shows that there are definable trees $S, T$ in $V[g]$ such that $p[S]=\mathbb{R}^{\#}$ and $p[T]=\mathbb{R} \backslash \mathbb{R}^{\#}$ in $V[\bar{h}]$. These trees belong to $V, V[g]$, and $V[G][g]$ by homogeneity and it can be argued that $p[S]=\mathbb{R}^{\#}$ in the sense of each model. Thus there is a fully elementary embedding ${ }^{5}$

$$
\pi: L(\mathbb{R})^{V[g]} \rightarrow L(\mathbb{R})^{V[G][g]} .
$$

We also have that from the perspective of $V[g]$, every set of reals in $L(\mathbb{R})$ is $\omega_{1}^{+}$-Universally Baire and hence $\mathbb{B}$-Universally Baire as $2^{\omega_{1}}=$ $\omega_{2}$.

\footnotetext{
${ }^{5}$ The argument of this paragraph can be recast using mice; essentially we are crossing the weak gap $\left(\delta_{1}^{2}, \theta\right)^{L(\mathbb{R})}$, and we can do it using the techniques of Lemma 66 below.
} 
Now suppose $\pi$ is such an embedding and fix $\alpha$ less than the $\theta$ of $L(\mathbb{R})^{V[g]}$. There is a prewellordering $\preceq \in L(\mathbb{R})^{V[g]}$ (with associated equivalence relation $\simeq$ ) of length $\alpha$ in $V[g]$, together with $S, T$ such that

(1) $p[S]=\preceq$ in $V[g]$

(2) $p[S]=\pi(\preceq)$ in $V[G][g]$

(3) $p[S]=\mathbb{R} \backslash p[T]$ in $V[g][G]$.

Since $\pi(\alpha)$ is the length of $\pi(\preceq)$ we must show that $p[S]^{V[G][g]}$ has length $\alpha$. This follows from the Foreman-Magidor theorem 34 .

The preceding remarks suggest that we add the existence of approximations to such an embedding to the induction hypothesis. This was how [26] came to formulate $I_{\alpha}$. In the next section we shall use $W_{\alpha}^{*}$ and $I_{\alpha}$ to get $I_{\alpha+\omega}$. If $\alpha$ is not in the range of $\pi_{N S}$ this will give $W_{\alpha+\omega}^{*}$ easily. Otherwise the arguments of section 3 will produce the required witnessing structures.

5.2. $L(\mathbb{R})$ and Cohen Forcing. Recall that the ordinal height of the transitive structure $J_{\alpha}(\mathbb{R})$ is $\omega \alpha$, and that the new sets of reals appearing in $J_{\alpha+1}(\mathbb{R})$ are precisely the new sets which are first order definable over $J_{\alpha}(\mathbb{R})$, that is

$$
P(\mathbb{R}) \cap J_{\alpha+1}(\mathbb{R})=P(\mathbb{R}) \cap \sum_{\omega}^{1}\left(J_{\alpha}(\mathbb{R})\right) .
$$

We say that $\alpha$ begins a gap if there is no $\beta<\alpha$ with $J_{\beta}(\mathbb{R})$ a $\Sigma_{1}$ elementary (with real parameters) submodel of $J_{\alpha}(\mathbb{R})$.

Lemma 36. Suppose $h \subset \operatorname{Col}(\omega, \kappa)$ is $V$-generic and $\alpha$ begins a gap in $L(\mathbb{R})^{V[h]}$. Suppose $J_{\alpha}(\mathbb{R})^{V[h]}=\mathrm{AD}$; then there is a unique pair $\alpha_{0}, \psi$ such that $\alpha_{0}$ that begins a gap in $L(\mathbb{R})$, and

$$
\psi: J_{\alpha_{0}}(\mathbb{R}) \rightarrow J_{\alpha}(\mathbb{R})^{V[h]}
$$

is $\Sigma_{1}$ elementary.

Proof. There are uniformly $\Sigma_{1}$ definable functions

$$
f_{\alpha}:[\omega \alpha]^{<\omega} \times \mathbb{R} \rightarrow J_{\alpha}(\mathbb{R})
$$

which are surjective. Using these we define a $\Sigma_{1}$ function $F$ as follows. Given a real $x$, decode a sequence $\left(x_{0}, \ldots, x_{n}\right)$ of reals and a real $y$ and suppose there is a finite sequence $t$ such that

$$
J_{\alpha}(\mathbb{R}) \models \phi_{y(0)}\left(\left(x_{0}, \ldots, x_{n}\right), f_{\alpha}(t, \hat{y})\right)
$$

where $\hat{y}(n)=y(n+1)$ and $\left(\phi_{k} \mid k<\omega\right)$ enumerates $\Sigma_{1}$ formulae with two free variables. Let $t^{*}$ be the Brouwer-Kleene least such $t$ and set 
$F(x)=f_{\alpha}\left(t^{*}, \hat{y}\right) . F$ is a uniformly $\Sigma_{1}$ partial map and if $\alpha$ begins a gap then $F$ as defined over $J_{\alpha}(\mathbb{R})$ is surjective. Let $M=F\left[\mathbb{R}^{V}\right]$ where $F$ is computed in $J_{\alpha}(\mathbb{R})$ of $V[g]$. By the homogeneity of $\operatorname{Col}(\omega, \kappa), \mathbb{R} \cap M=$ $\mathbb{R}^{V}$. Because $\left(\Sigma_{1}^{J_{\alpha}(\mathbb{R})}\right)^{V[h]}$ has the scale property, $M \prec_{\Sigma_{1}} J_{\alpha}(\mathbb{R})^{V[h]}$. It follows that $M \simeq J_{\alpha_{0}}(\mathbb{R})^{V}$ for some ordinal $\alpha_{0}$, and the inverse of the collapse is the desired map $\psi$.

Lemma 37. Suppose $\alpha$ is an inadmissible limit ordinal which begins a gap.

(1) There is a surjective function $f: \mathbb{R} \rightarrow J_{\alpha}(\mathbb{R})$ which is $\Delta_{1}$ definable over $J_{\alpha}(\mathbb{R})$ from a real $z_{0}$.

(2) If $A \in P(\mathbb{R}) \cap J_{\alpha+1}(\mathbb{R})$ then $A$ is projective in a set $D \in P(\mathbb{R})$ which is $\Delta_{1}$ definable over $J_{\alpha}(\mathbb{R})$ from a real.

(3) If $\triangle_{2 k+1}\left(J_{\alpha}(\mathbb{R})\right)$-determinacy holds then the pointclasses

$$
\prod_{\sim 2 k+2}\left(J_{\alpha}(\mathbb{R}) \text { and } \sum_{2 k+3}^{J_{\alpha}(\mathbb{R})}\right.
$$

have the scale property.

Proof. Inadmissibility of $J_{\alpha}(\mathbb{R})$ together with a Skolem hull argument gives a $\sum_{1}^{J_{\alpha}(\mathbb{R})}$ map $g: \mathbb{R} \rightarrow \omega \alpha$ which is cofinal. Using the uniform $\Sigma_{1}$ Skolem function this can be turned into the desired map $f$. For (2) note that every such $A$ can obtained from a $\Delta_{1}$ set of the form

$$
D=\left\{\left(x, x_{1}, \ldots, x_{k}\right) \in \mathbb{R}^{k+1} \mid J_{\alpha}(\mathbb{R}) \models \phi\left(x, f\left(x_{1}\right), \ldots, f\left(x_{k}\right), f(r)\right)\right\}
$$

by taking projections and complements, for some $\Sigma_{0}$ formula $\phi$ and real $r$. Part (3) follows from the second periodicity theorem. See [23] for further details.

Let $g \times G$ be $V$-generic over the product $\operatorname{Col}\left(\omega, \omega_{1}\right) \times P\left(\omega_{1}\right) / N S$. In order to relate $L(\mathbb{R})^{V[g]}$ to $L(\mathbb{R})^{V[g][G]}$, we must at the same time relate $L(\mathbb{R})^{V[G]}$ to $L(\mathbb{R})^{V[G][g]}$. Now $V[G][g]$ comes from $V[G]$ by adding just one Cohen real. The following lemma, due to Woodin and H. Friedman, establishes the level-by-level relationship between the two $L(\mathbb{R})$ 's that we need. See [8] for other results in this vein.

Lemma 38. Suppose $J_{\alpha}(\mathbb{R}) \models A D$ and $\alpha$ begins a gap. Suppose $g \subset \operatorname{Col}(\omega, \omega)$ is Cohen generic over $V$; then there exists a unique $\Sigma_{1}$ elementary embedding

$$
j: J_{\alpha}(\mathbb{R}) \rightarrow J_{\alpha}(\mathbb{R})^{V[g]}
$$

such that

$$
j \uparrow \alpha=\text { identity. }
$$

Furthermore, if all $\sum_{1}^{J_{\alpha}(\mathbb{R})}$ sets of reals have the Baire property, then $j$ is $\Sigma_{2}$-elementary. 
Proof. The reader of [8][section 5] will easily adapt it so as to obtain this localization. Nevertheless, we sketch a proof for the sake of completeness.

We think of the reals as $\omega^{\omega}$. For $p \in \omega^{<\omega}$ let $N_{p}$ denote the neighborhood determined by $p$. Let $\mathcal{B}$ denote the $\sigma$-algebra of sets of reals in $L(\mathbb{R})$ which have the Baire property. Let $\mathbb{B}$ denote the quotient algebra $\mathcal{B} / I$ where $I$ is the ideal of meager sets. Clearly the map

$$
\pi: \operatorname{Col}(\omega, \omega) \rightarrow \mathbb{B}
$$

be defined by $\pi(p)=\left[N_{p}\right]$ is a dense embedding so if $g \subset \operatorname{Col}(\omega, \omega)$ is $V$-generic then $g$ induces an ultrafilter $U_{g}$ on $B$. That is, a set $A$ is in $U_{g}$ if and only if $A \cap N_{p}$ is comeager in $N_{p}$ for some $p \in g$.

In $V[g]$ we form the ultrapower $U l t\left(J_{\alpha}(\mathbb{R}), U_{g}\right)$ using functions belonging to $J_{\alpha}(\mathbb{R})$. We may assume that these functions are total with domain $\mathbb{R}$. We first show that $\operatorname{Ult}\left(J_{\alpha}(\mathbb{R}), U_{g}\right)$ is well-founded. Assume a condition $p$ forces that $\left(\left[\dot{f}_{n}\right]\right)$ is a decreasing sequence in the ultrapower. For $s \in \omega^{<\omega}$ define a condition $p_{s} \in \operatorname{Col}(\omega, \omega)$, a set $A_{s}$, and a function $f_{s}$ all in $J_{\alpha}(\mathbb{R})$ such that

(a) $p \subseteq p_{\emptyset}$

(b) $\left\{p_{s \frown n} \mid n \in \omega\right\}$ is a maximal antichain below $p_{s}$

(c) $A_{s} \subset N_{s}$ is comeager in $N_{s}$,

(d) $p_{s} \Vdash_{C o l(\omega, \omega)} \dot{f}_{l h(s)}=f_{s}$.

(e) $s \subset t, s \neq t$, and $x \in A_{t}$ implies $f_{t}(x) \in f_{s}(x)$.

By the Baire Category Theorem

$$
\bigcap_{n<\omega} \bigcup_{l h(s)=n} A_{s} \neq \emptyset
$$

so there are $x, h \in \omega^{\omega}$ so that $x \in A_{h\lceil n}$ for every $n<\omega$ and hence $\left\{f_{h \uparrow n}(x) \mid n<\omega\right\}$ is an $\in$-decreasing sequence, giving the desired contradiction. Thus the ultrapower has a transitivization $M$.

Los' theorem for $\Sigma_{0}$ formulae comes from almost-everywhere uniformization:

Claim. Suppose $A \subset \mathbb{R} \times \mathbb{R}$ and $A \in J_{\alpha}(\mathbb{R})$; then there is a continuous function $f$ and a comeager set $D \subseteq \operatorname{dom}(f)$ such that if $x \in D$ and there is $y$ such that $(x, y) \in A$ then $(x, f(x)) \in A$.

This standard result is proved by unfolding the Banach-Mazur game. It yields

Claim. For any functions $f_{1}, \ldots, f_{n} \in J_{\alpha}(\mathbb{R})$ and $\Sigma_{0}$ formula $\phi$,

$$
\operatorname{Ult}\left(J_{\alpha}(\mathbb{R}), U_{g}\right) \models \phi\left(\left[f_{1}\right], \ldots,\left[f_{n}\right]\right)
$$


if and only if

$$
\left\{r \in \mathbb{R} \mid J_{\alpha}(\mathbb{R}) \models \phi\left(f_{1}(r), \ldots, f_{n}(r)\right)\right\} \in U_{g} .
$$

This follows by the usual induction. For the existential step, we may assume that $\beta<\alpha$ begins a gap and $J_{\beta}(\mathbb{R}) \models \exists Y \phi(Y, f(x))$, for all $x$ in some set $B \in U_{g}$. Let $F \in J_{\alpha}(\mathbb{R})$ map $\mathbb{R}$ onto $J_{\beta}(\mathbb{R})$. Let $A$ denote the set of $(x, y)$ such that $\phi(F(y), f(x))$ holds in $J_{\beta}(\mathbb{R})$, let $h^{*}$ uniformize this $A$ as in the claim, and set $h(x)=F\left(h^{*}(x)\right)$. By induction, $\phi([h],[f])$ holds in the ultrapower, as desired.

It follows at once that the ultrapower map $j: J_{\alpha}(\mathbb{R}) \rightarrow M$ is $\Sigma_{1}$ elementary. We leave it to the reader to check that the standard terms for reals correspond to continuous functions on $\mathbb{R}$, and thus $\mathbb{R}^{M}=\mathbb{R}^{V[g]}$. It follows that $M=J_{\gamma}(\mathbb{R})^{V[g]}$, for some $\gamma$.

In $J_{\alpha}(\mathbb{R})$ any well-ordered union of meager sets is meager. It follows that for any ordinal $\eta$ and function $f: \mathbb{R} \rightarrow \eta$ in $J_{\alpha}(\mathbb{R})$ there is a dense set $D \subset \operatorname{Col}(\omega, \omega)$ such that $f$ is constant on a comeager subset of $N_{p}$ for any $p \in D$. It follows that $\gamma=\alpha$ and that $j \uparrow \omega \alpha$ is the identity.

Finally, suppose all $\sum_{1}^{J_{\alpha}(\mathbb{R})}$ sets of reals have the Baire property. We show that Los' theorem holds for $\Sigma_{1}$ formulae, so that $j$ is $\Sigma_{2}$ elementary, as desired. So suppose $\phi$ is $\Sigma_{1}$, and

$$
J_{\alpha}(\mathbb{R}) \models \exists Y \phi(Y, f(x)),
$$

for comeager many $x$ in $N_{p}$, where $p \in g$. Say this holds for all $x \in B$, where $B$ is Borel and comeager in $N_{p}$. Let us assume $\alpha$ is a limit ordinal for simplicity; otherwise we use Jensen's $S$-hierarchy. For $\beta<\alpha$, put

$$
x \in B_{\beta} \Leftrightarrow J_{\beta}(\mathbb{R}) \models \exists Y \phi(Y, f(x)) .
$$

The prewellordering $x_{1} \leq x_{2}$ iff $\mu \beta\left(x_{1} \in B_{\beta}\right) \leq \mu \beta\left(x_{2} \in B_{\beta}\right)$ is $\Sigma_{1}$ over $J_{\alpha}(\mathbb{R})$, so it has the Baire property, and thus by Kuratowski-Ulam some $B_{\beta}$ is nonmeager in $N_{p}$, and thus comeager on some $N_{q}$ with $p \subseteq q$. By density, there is such a $\beta, q$ with $q \in g$. We can now find the desired witness function $h$ for $\phi, f$ so that $h \in J_{\beta+1}(\mathbb{R})$.

The following lemma shows one way we shall use these results.

Lemma 39. Assume $I_{\alpha}$ holds, and that $\alpha$ begins a gap in $V^{\operatorname{Col}\left(\omega, \omega_{1}\right)}$. Let $h \times H$ be $\operatorname{Col}\left(\omega, \omega_{1}\right) \times\left(P\left(\omega_{1}\right) / N S\right)^{V}$-generic over $V$, and suppose $V[h] \models W_{\alpha}^{*}$; then

(1) there is a unique $\Sigma_{1}$ embedding from $J_{\alpha}(\mathbb{R})^{V[H]}$ to $J_{\alpha}(\mathbb{R})^{V[H][h]}$ that fixes all ordinals,

(2) there is a unique ordinal $\alpha_{0}$ that begins a gap in $V$, and $\Sigma_{1}$ embedding from $J_{\alpha_{0}}(\mathbb{R})^{V}$ to $J_{\alpha}(\mathbb{R})^{V[h]}$.

Thus $\mathrm{AD}$ holds in $J_{\alpha_{0}}(\mathbb{R})^{V}$, in $J_{\alpha}(\mathbb{R})^{V[h]}$, in $J_{\alpha}(\mathbb{R})^{V[H]}$, and in $J_{\alpha}(\mathbb{R})^{V[H][h]}$. 
Proof. We have AD in $J_{\alpha}(\mathbb{R})^{V[h]}$ and $J_{\alpha}(\mathbb{R})^{V[H][h]}$ by Lemma 10 . Moreover, the embedding given by $I_{\alpha}$ shows that $\alpha$ begins a gap in $V[H][h]$.

$V[H][h]$ is a $\operatorname{Col}(\omega, \omega)$ extension of $V[H]$, so Lemma 36 gives a unique $\Sigma_{1}$ embedding from $J_{\gamma}(\mathbb{R})^{V[H]}$ to $J_{\alpha}(\mathbb{R})^{V[H][h]}$, for some $\gamma$ that begins a gap in $V[H]$. But then $\mathrm{AD}$ holds in $J_{\gamma}(\mathbb{R})^{V[H]}$, so we can apply our lemma 38 to see that $\gamma=\alpha$, and the embedding is the identity on the ordinals.

Finally, $\alpha_{0}$ and the embedding of (2) are just what we get when we apply 36 to the $\operatorname{Col}\left(\omega, \omega_{1}\right)$-extension from $V$ to $V[h]$.

5.3. The Baire property for $\sum_{1}^{J_{\alpha}(\mathbb{R})}$. We present a sufficient condition for the pointclass $\Gamma=\sum_{1}^{J_{\alpha}(\mathbb{R})}$ to have the Baire property. The condition is implicit in Solovay's theorems on the Baire property for $\Sigma_{2}^{1}$ sets. We assume that $\alpha$ begins a gap, $J_{\alpha}(\mathbb{R}) \models \mathrm{AD}$, and $\operatorname{cof}(\alpha)>\omega^{6}{ }^{6}$ We define

$$
C_{\Gamma}(x)=\mathbb{R} \cap O D_{x}^{<\alpha},
$$

that is, for reals $x, y$ we put $y \in C_{\Gamma}(x)$ if there is $\beta<\alpha$ such that $y$ is ordinal definable over $J_{\beta}(\mathbb{R})$ from the parameter $x{ }^{7}$ Similarly

$$
C_{\Gamma}(a)=P(a) \cap O D_{a \cup\{a\}}^{J_{\alpha}(\mathbb{R})}
$$

for a countable transitive set $a$. Because we have $W_{\alpha}$,

$$
C_{\Gamma}(a)=\operatorname{Lp}^{\Gamma}(a)
$$

is the result of stacking all mice projecting to $a$ and having $\omega_{1}$-iteration strategies belonging to $J_{\alpha}(\mathbb{R})$. A transitive set $M$ is $\Gamma$-closed if $a \in M$ implies $C_{\Gamma}(a) \in M$. The model $L^{\Gamma}[x]$ is the minimal transitive model of height $\omega_{1}$ which contains $x$ and is $\Gamma$-closed. Finally, we say that $\omega_{1}$ is $\Gamma$-inaccessible to reals iff $C_{\Gamma}(x)$ is countable for all reals $x$, or equivalently,

$$
\omega_{1}^{L^{\Gamma}[x]}<\omega_{1}
$$

for all reals $x$.

The classical argument of Solovay in the case $\Gamma=\Sigma_{2}^{1}$ yields

Lemma 40. Assume $J_{\alpha}(\mathbb{R}) \models A D, \alpha$ begins a gap, $\operatorname{cof}(\alpha)>\omega$ and $\omega_{1}$ is $\Gamma$-inaccessible to reals where $\Gamma=\sum_{\sim}^{J_{\alpha}(\mathbb{R})} \cap P(\mathbb{R})$; then

(1) $\Gamma$ has the Baire Property.

(2) $\omega_{1}$ is $\Gamma$-inacessible to reals in $V[g]$ whenever $g$ is Cohen generic over $V$.

\footnotetext{
${ }^{6}$ In the case that $\operatorname{cof}(\alpha)=\omega$ or $\alpha$ is a successor, the facts that the class of sets with the Baire property is closed under countable unions is enough for us.

${ }^{7}$ If $J_{\alpha}(\mathbb{R})$ is admissible, then $C_{\Gamma}$ is the largest thin $\Gamma$ set, but we don't know whether this is true in general.
} 
Proof. Let $A$ be a set in $\Gamma$. Let $x$ be a real such that $A$ is $\Sigma_{1}$-definable over $J_{\alpha}(\mathbb{R})$ from $x$. Let $N$ be a rank initial segment of $L^{\Gamma}[x]$ containing its reals. There are comeager many Cohen generics over $N$ so if each one lands in $\mathbb{R} \backslash A$ we're finished. Assume therefore that there is $g_{0}$ which is Cohen generic over $N$ with $g_{0} \in A$. Let $\beta<\alpha$ be least such that $J_{\beta}(\mathbb{R}) \models g_{0} \in A$. Let $\beta_{1}=\beta+\omega$ and let $T$ be the tree of the scale on the universal $\Sigma_{1}\left(J_{\beta_{1}}(\mathbb{R})\right)$ set. Note that $N$ is a rank initial segment of $L[T, N]$. Now assume toward a contradiction that there exists a sequence of open dense sets $D_{n} \subseteq \mathbb{R}$ such that

$$
g \in \bigcap_{n \in \omega} D_{n} \Rightarrow g \in(\neg A)^{J_{\beta}(\mathbb{R})} .
$$

Then for any real $z$ coding $N$, the model $J_{\beta_{1}}(\mathbb{R})$ satisfies the sentence asserting the existence of $g_{0},\left\{D_{n} \mid n \in \omega\right\}$ and $\beta$ satisfying

(1) $g_{0}$ is Cohen generic over the model coded by $z$

(2) $g_{0} \in A^{J_{\beta}(\mathbb{R})}$

(3) $g \in \bigcap_{n \in \omega} D_{n} \Rightarrow g \in(\neg A)^{J_{\beta}(\mathbb{R})}$

This is a $\Sigma_{1}$ sentence and so it holds in $L[T, z]$ by absoluteness. Thus for every $g$ which is $L[T, z]$-generic, $g \in(\neg A)^{J_{\beta}(\mathbb{R})}$. Now, $g_{0}$ is generic over $L[T, N]$. Let $G$ be generic over $L[T, N]\left[g_{0}\right]$ for $\operatorname{Col}(\omega, N)$. Then $g_{0}$ is generic over $L[T, N][G]$. But $G$ codes $N$ which is a contradiction. Thus by the Baire Property there is a condition $p$ such that $g \in A^{J_{\beta}(\mathbb{R})}$ for comeager many $g$ below $p$. Let $O$ be the union of all neighborhoods which have this property. We claim that $A \backslash O$ is meager. Otherwise there is an $L^{\Gamma}[x, y]$ generic $g$ which lands in $A \backslash O$ where $y$ codes the opern set $O$. But $A \backslash O$ is $\Sigma_{1}$ so the preceeding analysis produces a $q$ such that comeager many $g$ below $q$ land in $A \backslash O$, a contradiction as $N_{q} \subseteq O$. For part (2) note that we interpret $\Gamma$ in $V[g]$ as $\left(\Sigma_{1}\left(J_{\alpha}(\mathbb{R})\right) \cap\right.$ $P(\mathbb{R}))^{V[g]}$. Let $\tau$ be a standard term for a real. It suffices to show that $C_{\Gamma}\left(\tau_{g}\right)$ is countable. Assume otherwise. Thus we may assume that there is a single $\Sigma_{1}$ formula $\psi(x, y, z)$ and a condition $p$ which forces that $\left\{r_{\eta} \mid \eta<\omega_{1}\right\}$ is a distinct sequence of reals where

$$
r_{\eta}=\left\{n<\omega \mid J_{\alpha}(\mathbb{R})^{V[g]} \models \psi\left(\eta, n, \tau_{g}\right)\right\} .
$$

Let $z$ be a real coding $\tau$ and let $\phi(\eta, q, n)$ be the $\Sigma_{1}$ formula which asserts that $q \leq p, z$ codes a term $\tau$ and

$$
q \Vdash_{C o l(\omega, \omega)} \psi(\eta, n, \tau) .
$$

Setting $r_{\eta}^{*}=f[\{(q, n) \mid \phi(\eta, q, n)\}]$ where $f: \operatorname{Col}(\omega, \omega) \times \omega \rightarrow \omega$ is a fixed bijection we see that each $r_{\eta}^{*}$ belongs to $C_{\Gamma}(z)$ and that they are distinct, contradicting our hypotheses. 


\section{The Induction Step in the Inadmissible Case}

We now handle the induction step in the uncountable cofinality, inadmissible case. This was the case labelled (2)(b) in case breakdown 2 . The countable cofinality inadmissible case (2)(a) is similar but simpler, so we omit it. The general successor case (1) involves no ideas beyond those in section 3, so again, we omit it.

We assume for the rest of this section that NS is saturated, and $\operatorname{WRP}_{(2)}\left(\omega_{2}\right)$ holds. We fix for the remainder of the section an $\alpha$ such that $I_{\alpha}$ holds, and for some (equivalently all) $g$ generic over $\operatorname{Col}\left(\omega, \omega_{1}\right)$ :

(1) $V[g] \models W_{\alpha}^{*}$, and

(2) $V[g] \models \alpha$ has uncountable cofinality, and $J_{\alpha}(\mathbb{R})$ is inadmissible. Our goal is to prove that $I_{\alpha+1}$ holds, and that $W_{\alpha+1}^{*}$ holds in $V[g]$.

6.1. The map $\pi_{N S} \uparrow \alpha_{0}$. If $G$ is any $V$-generic over $P\left(\omega_{1}\right) / N S$, then we let

$$
\pi_{G}: V \rightarrow \operatorname{Ult}(V, G)
$$

be the canonical embedding into the generic ultrapower. One byproduct of the proof of our main theorem is that $\pi_{G} \uparrow \Theta^{L(\mathbb{R})}$ is independent of $G$. As we go along, we are showing that $\pi_{G}(\gamma)$ is independent of $G$ for certain $\gamma$. In particular, for any $\operatorname{Col}\left(\omega, \omega_{1}\right)$-generic $g$, let $\alpha_{0}$ be the ordinal beginning a gap in $V$ that is given by 39 , and

$$
\tau^{g}: J_{\alpha_{0}}(\mathbb{R})^{V} \rightarrow J_{\alpha}(\mathbb{R})^{V[g]}
$$

the unique $\Sigma_{1}$ map. By homogeneity, $\tau^{g} \uparrow\left(\mathbb{R} \times \omega \alpha_{0}\right)$ is independent of $g$, and so we may omit the superscript $g$ when we are only concerned with this part of $\tau^{g}$. Of course, $J_{\alpha_{0}}(\mathbb{R})$ is the $\Sigma_{1}$ hull of $\left(\mathbb{R} \times \omega \alpha_{0}\right)$. We have

Lemma 41. Let $G$ be $P\left(\omega_{1}\right) / N S$-generic over $V$; then

(a) $\pi_{G} \uparrow \omega \alpha_{0}=\tau \uparrow \omega \alpha_{0}$,

(b) $\pi_{G}\left(\alpha_{0}\right) \geq \alpha$, and

(c) if $\pi_{G}\left(\alpha_{0}\right)=\alpha$, then for all $P\left(\omega_{1}\right) / N S$ generic $H, \pi_{H}\left(\alpha_{0}\right)=\alpha$.

Proof. Let $g$ be such that $g \times G$ is $\operatorname{Col}\left(\omega, \omega_{1}\right) \times P\left(\omega_{1}\right) / N S$ generic. Let $\pi$ witness $I_{\alpha}$, and let

$$
\psi: J_{\alpha}(\mathbb{R})^{V[G]} \rightarrow J_{\alpha}(\mathbb{R})^{V[G][g]}
$$

be the Cohen ultrapower map. $\psi$ is $\Sigma_{1}$ elementary, and the identity on ordinals. We have then

$$
\tau^{g}=\pi^{-1} \circ \psi \circ\left(\pi_{H} \uparrow J_{\alpha_{0}}(\mathbb{R})^{V}\right)
$$


because both sides yield $\Sigma_{1}$ embeddings of $\left.J_{\alpha_{0}}(\mathbb{R})^{V}\right)$ into $J_{\alpha_{0}}(\mathbb{R})^{V[h]}$. Since $\psi$ and $\pi$ are the identity on ordinals, we have (a).

For (b), let $\beta$ be least such that $\pi_{G}(\beta) \geq \alpha$, and suppose $\alpha_{0}<\beta$ toward contradiction. Let $\gamma$ end the gap in $V$ that begins with $\alpha_{0}$. If $\beta \leq \gamma$, then $\left[\pi_{G}\left(\alpha_{0}\right), \pi_{G}(\beta)\right]$ is a gap in $V[G]$ by the elementarity of $\pi_{G}$, and $\alpha$ is inside it, so $\alpha$ does not begin a gap in $V[G]$, contradiction. Suppose $\gamma<\beta$. Let $x$ be a real in $V$ and $\varphi(v)$ a $\Sigma_{1}$ formula such that $J_{\gamma+1}(\mathbb{R})^{V} \models \varphi[x]$, but $J_{\alpha_{0}}(\mathbb{R}) \forall=\varphi[x]$. Using $\tau^{g}=\pi^{-1} \circ \psi \circ$ $\left(\pi_{G}\left\lceil J_{\alpha_{0}}(\mathbb{R})^{V}\right)\right.$, we see that $J_{\alpha}(\mathbb{R})^{V[g]} \models \varphi[x]$. But this contradicts the elementarity of $\tau^{g}$.

For (c), we show first that all $\left(\sum_{1}^{J_{\alpha}(\mathbb{R})}\right)^{V[G]}$ sets have the Baire property. Let $\Gamma=\left(\Sigma_{1}^{J_{\alpha}(\mathbb{R})}\right)^{V[G]}$. We wish to show that in $V[G], \omega_{1}$ is $\Gamma$-inaccessible to reals. Letting $\Gamma_{0}=\left(\Sigma_{1}^{J_{\alpha_{0}}(\mathbb{R})}\right)^{V}$, it is enough to show that in $V, \omega_{1}$ is $\Gamma_{0}$-inaccessible to reals. If not, there is in $V$ a real $x$ such that every countable ordinal is the order type of a wellorder in $C_{\Gamma_{0}}(x)$. Applying $\pi_{G}$ to this fact, we have $y \in C_{\Gamma}(x)^{V[G]}$ coding a wellorder of $\omega$ of order type $\omega_{1}^{V}$. Applying $\pi^{-1} \circ \psi$ to this fact, we see that $y \in V[g]$, and $y$ is ordinal definable in $V[g]$ from $x$. But then $y \in V$, a contradiction.

Having shown $\pi_{G}(\gamma)$ independent of $G$, we may use $\pi_{N S}(\gamma)$ to denote the common value of all $\pi_{G}(\gamma)$. So at the moment, $\pi_{N S} \uparrow \alpha_{0}$ is determined, and it is the Skolem hull map $\tau$.

Let us say that a pointclass has the Baire property just in case all sets of reals belonging to it have the Baire property. Our proof of the last lemma gives

Lemma 42. Let $g \times G$ be $\operatorname{Col}\left(\omega, \omega_{1}\right) \times P\left(\omega_{1}\right) / N S$-generic; then

(1) if $\pi_{H}\left(\alpha_{0}\right)=\alpha$, then $\left(\sum_{1}^{J_{\alpha_{0}}(\mathbb{R})}\right)^{V}$ has the Baire property, and

(2) in any case, each of the pointclasses

(a) $\left(\sum_{1}^{J_{\alpha}(\mathbb{R})}\right)^{V[G]}$

(b) $\left(\sum_{1}^{J_{\alpha}(\mathbb{R})}\right)^{V[G][g]}$, and

(c) $\left(\sum_{1}^{J_{\alpha}(\mathbb{R})}\right)^{V[g]}$

has the Baire property in its respective model.

Proof. We showed (1) in proving the last lemma.

(2)(a) follows from (1) in the case that $\pi_{G}\left(\alpha_{0}\right)=\alpha$ by elementarity. But if $\pi_{G}\left(\alpha_{0}\right)>\alpha$, then $\mathrm{AD}$ in $J_{\alpha_{0}}(\mathbb{R})^{V}$ gives us $\mathrm{AD}$, and hence the Baire property, for all sets in $J_{\alpha+1}(\mathbb{R})^{V[G]}$. So this gives $(2)$ (a) in any case.

We get (2)(b) from (2)(a) and part (2) of Lemma 40 . 
For $(2)(\mathrm{c})$, we show that $\omega_{1}$ is $\Gamma$-inaccessible to reals in $V[g]$, where $\Gamma=\left(\Sigma_{1}^{J_{\alpha}(\mathbb{R})}\right)^{V[g]}$. If not, there is a real $x \in V[g]$ such that every countable ordinal has a counting that is $\operatorname{OD}(x)$ over some $J_{\beta}(\mathbb{R})^{V[g]}$, where $\beta<\alpha$. Applying $\pi^{g}$, we have that $\omega_{1}$ is not $\left.\left(\Sigma_{1}^{J_{\alpha}(\mathbb{R})}\right)^{V[G][g]}\right]_{-}$ inaccessible to reals in $V[G][g]$, contrary to $(2)(\mathrm{b})$.

6.2. The plan. The following diagram helps illustrate the steps of our proof.

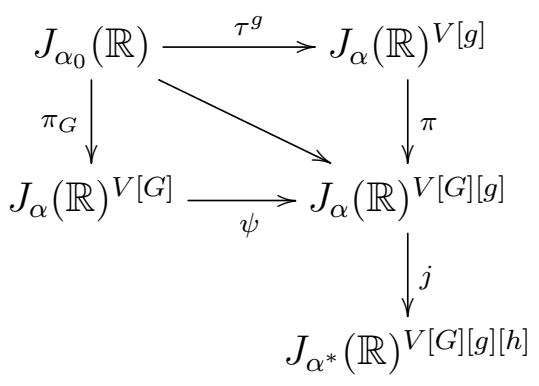

Here $g \times G$ is an arbitrary $\operatorname{Col}\left(\omega, \omega_{1}\right) \times P\left(\omega_{1}\right) / N S$ generic, and $h \subset$ $\operatorname{Col}\left(\omega, \omega_{2}^{V}\right)$ is $V[G][g]$-generic. Of course, $V[G][g][h]$ could be reorganized as $V[\bar{h}]$ for another $\operatorname{Col}\left(\omega, \omega_{2}^{V}\right)$-generic. $J_{\alpha}(\mathbb{R})^{V[G]}$ and $J_{\alpha}(\mathbb{R})^{V[G][g]}$ are connected via the Cohen ultrapower map $\psi$. The map $\pi$ is given by $I_{\alpha}$, and $\tau^{g}$ is the $\Sigma_{1}$ hull map of 36 . It may be that $\alpha_{0}$ is a discontinuity point of $\pi_{G}$, so in general, $\pi_{G}$ is only $\Sigma_{1}$ elementary as a map from $J_{\alpha_{0}}(\mathbb{R})$ to $J_{\alpha}(\mathbb{R})^{V[G]}$. If $\pi_{G}\left(\alpha_{0}\right)=\alpha$, then the map is fully elementary.

Our first burden is to define an appropriate level $\alpha^{*}$ of the $L(\mathbb{R})$ of $V^{\operatorname{Col}\left(\omega, \omega_{2}\right)}$ and to construct $j$. We shall show that $\alpha^{*}$ and $j \uparrow \omega \alpha$ are independent of $g, G$ and $h$, using the fact that $\alpha$ has a "name" provided by any witness to its inadmissibility in $V[g]$. We will use $j$ and $\alpha^{*}$ to show that $\pi$ is in fact $\Sigma_{2}$-elementary.

The proof then breaks into cases. If $\pi_{G}\left(\alpha_{0}\right)=\alpha$ for some (equivalently all) $G$, then $\alpha_{0}$ is inadmissible and begins a gap in $L(\mathbb{R})^{V}$, so we have from [22] a natural mouse operator $J$ on $H\left(\omega_{1}\right)^{V}$ coding up $\Sigma_{1}$ truth over $J_{\alpha_{0}}(\mathbb{R})$. We can extend $J$ to $H\left(\omega_{2}\right)$ using $\pi_{G}$ and $\pi$; at this point we use $j$, and the fact that $\pi$ is $\Sigma_{2}$-elementary, to see that $\pi_{G}(J)\left\lceil H\left(\omega_{2}\right)^{V}\right.$ is independent of $G$. By Mouse Reflection, $J$ extends to $H\left(\omega_{3}\right)^{V}$. We can now use the core model theory of Theorem 28 to get the mice needed for $W_{\alpha+1}^{*}$ in $V[g]$, and to prove $I_{\alpha+1}$.

If $\pi_{G}\left(\alpha_{0}\right)>\alpha$, then since $V \models W_{\alpha_{0}}^{*}, V[G] \models W_{\alpha+1}^{*}$, so $V[G][g] \models$ $W_{\alpha+1}^{*}$ by the elementarity of the Cohen ultrapower. So we don't need core model theory to get the new mice, but we do need to get them 
in $V[g]$, rather than in $V[G][g]$. This is done with an induction that shows at the same time that $\pi$ and $j$ are fully elementary.

6.3. Lifting $J_{\alpha}(\mathbb{R})^{V[G][g]}$ to $V^{\operatorname{Col}\left(\omega, \omega_{2}\right)}$.

Lemma 43. There is a function $l: \omega_{1} \rightarrow\left(\alpha_{0}+1\right)$ such that whenever $G$ is $P\left(\omega_{1}\right) / N S$-generic over $V$, then $\alpha=[l]_{G}$.

Proof. Let $\left\{S_{\xi}, l_{\xi} \mid \xi<\omega_{1}\right\}$ be such that $\left\{S_{\xi} \mid \xi<\omega_{1}\right\}$ is a maximal antichain in $P\left(\omega_{1}\right) / N S, l_{\xi}: \omega_{1} \rightarrow\left(\alpha_{0}+1\right)$, and $S_{\xi}$ forces $l_{\xi}$ to represent $\alpha$ in the generic ultrapower. Define $l(\eta)=l_{\xi}(\eta)$ where $\xi$ is least such that $\eta \in S_{\xi}$. It's easy to see that $l$ is as desired.

We fix $l$ as in the lemma. By lemma 42, we may assume that for all $\beta, \sum_{1}^{J_{l(\beta)}(\mathbb{R})}$ has the Baire property.

Let $F$ be the uniform $\Sigma_{1}$ function for levels of $L(\mathbb{R})$, as described in the proof of 36 .

We use " $\forall * \sigma$ " to abbreviate " for a club of $\sigma \in\left[\omega_{2}\right]^{\omega}$ ". If $\sigma \in\left[\omega_{2}\right]^{\omega}$, then $\operatorname{otp}(\sigma)$ is the image of $\sigma$ under transitive collapse, that is, its order-type. For $a \subseteq \sigma^{<\omega}, a^{\sigma}$ is the image of $a$ under the collapse of $\sigma$.

Lemma 44. There is a term for an ordinal $\alpha^{*}$ such that $V^{\operatorname{Col}\left(\omega, \omega_{2}\right)}=\alpha^{*}$ begins a gap, and for any $p \in \operatorname{Col}\left(\omega, \omega_{2}\right)$, standard term for a real $\tau$, and $\Sigma_{1}$ formula $\phi$, the following are equivalent:

(1) $p \Vdash_{C o l\left(\omega, \omega_{2}\right)} J_{\alpha^{*}}(\mathbb{R}) \models \phi(F(\tau))$

$(2) \forall^{*} \sigma p^{\sigma} \Vdash_{C o l(\omega, o t p(\sigma))} J_{l\left(\sigma \cap \omega_{1}\right)}(\mathbb{R}) \models \phi\left(F\left(\tau^{\sigma}\right)\right)$

Proof. We define a name for a structure

$$
\dot{\mathcal{M}}=\left(\dot{M}, \dot{\in}_{\mathcal{M}}, \dot{=}_{\mathcal{M}}\right)
$$

as follows. For $p \in \operatorname{Col}\left(\omega, \omega_{2}\right)$ and $\tau, \bar{\tau}$ standard terms for reals

(a) $(p, \tau) \in \dot{M}$ if and only if

$$
\forall^{*} \sigma p^{\sigma} \Vdash_{C o l(\omega, o t p(\sigma))} J_{l\left(\sigma \cap \omega_{1}\right)}(\mathbb{R}) \models F\left(\tau^{\sigma}\right) \text { exists, }
$$

(b) $(p,(\tau, \bar{\tau})) \in \dot{\epsilon}_{\mathcal{M}}$ if and only if

$$
\forall^{*} \sigma p^{\sigma} \Vdash_{C o l(\omega, o t p(\sigma))} J_{l\left(\sigma \cap \omega_{1}\right)}(\mathbb{R}) \models F\left(\tau^{\sigma}\right) \in F\left(\bar{\tau}^{\sigma}\right),
$$

- (c) []$(p,(\tau, \bar{\tau})) \in \doteq_{\mathcal{M}}$ if and only if

$$
\forall^{*} \sigma p^{\sigma} \Vdash_{C o l(\omega, o t p(\sigma))} J_{l\left(\sigma \cap \omega_{1}\right)}(\mathbb{R}) \models F\left(\tau^{\sigma}\right)=F\left(\bar{\tau}^{\sigma}\right) .
$$

By $(\tau, \bar{\tau})$ above we really mean the term for the ordered pair. Notice that because $\sum_{1}^{J_{l(\beta)}(\mathbb{R})}$ has the Baire property, we can understand $p^{\sigma} \Vdash_{C o l(\omega, o t p(\sigma))} J_{l\left(\sigma \cap \omega_{1}\right)}(\mathbb{R}) \models \varphi(\tau)$ to mean that $\varphi\left(\tau^{h}\right)$ is true in $J_{l\left(\sigma \cap \omega_{1}\right)}(\mathbb{R})$, for comeager many generics $h$ such that $p^{\sigma} \in h$. We prove the following Los-type assertion. 
Claim 45. For a condition $p$, terms $\tau_{1}, \ldots, \tau_{k}$, and a $\Sigma_{1}$ formula $\phi$ the following are equivalent.

(1) $p \Vdash_{C o l\left(\omega, \omega_{2}\right)} \tau_{1}, \ldots, \tau_{k} \in \dot{M} \wedge \dot{\mathcal{M}} \models \phi\left(\tau_{1}, \ldots, \tau_{k}\right)$

(2) $\forall^{*} \sigma p^{\sigma} \Vdash_{C o l(\omega, o t p(\sigma))} J_{h\left(\sigma \cap \omega_{1}\right)}(\mathbb{R}) \models \phi\left(F\left(\tau_{1}^{\sigma}\right), \ldots, F\left(\tau_{k}^{\sigma}\right)\right)$.

Proof. Note that $p \Vdash_{C o l\left(\omega, \omega_{2}\right)} \tau \in \dot{M}$ if and only if $(p, \tau) \in \dot{M}$. The atomic cases follow easily. We handle negation as follows. Assume $\phi\left(\tau_{1}, \ldots, \tau_{k}\right)$ is $\neg \psi\left(\tau_{1}, \ldots, \tau_{k}\right), \psi$ is $\Sigma_{1}$, and the equivalence above holds for $\psi\left(\tau_{1}, \ldots, \tau_{k}\right)$. Assume first that $p \Vdash_{C o l\left(\omega, \omega_{2}\right)} \dot{\mathcal{M}}=\phi\left(\tau_{1}, \ldots, \tau_{k}\right)$. Assume toward a contradiction that there is a stationary set $A_{0} \subset\left[\omega_{2}\right]^{\omega}$ such that for $\sigma \in A$ we have

$$
\neg p^{\sigma} \Vdash_{C o l(\omega, o t p(\sigma))} J_{l\left(\sigma \cap \omega_{1}\right)}(\mathbb{R}) \models \phi\left(F\left(\tau_{1}^{\sigma}\right), \ldots, F\left(\tau_{k}^{\sigma}\right)\right) .
$$

Thus by refining $p^{\sigma}$ and pressing down we find a $q$ below $p$ and a stationary subset $A \subset A_{0}$ such that $\sigma \in A$ implies

$$
q^{\sigma} \Vdash_{C o l(\omega, o t p(\sigma))} J_{l\left(\sigma \cap \omega_{1}\right)}(\mathbb{R}) \models \psi\left(F\left(\tau_{1}^{\sigma}\right), \ldots, F\left(\tau_{k}^{\sigma}\right)\right) .
$$

Similarly we get a stationary set $B$ and a condition $r$ below $q$ such that for $\sigma \in B$ we have

$$
r^{\sigma} \Vdash_{C o l(\omega, o t p(\sigma))} J_{l\left(\sigma \cap \omega_{1}\right)}(\mathbb{R}) \models \phi\left(F\left(\tau_{1}^{\sigma}\right), \ldots, F\left(\tau_{k}^{\sigma}\right)\right) .
$$

We now find an ordinal $\gamma<\omega_{2}$ above $r$ so that $A \cap[\gamma]^{\omega}$ and $B \cap[\gamma]^{\omega}$ are both stationary in $[\gamma]^{\omega}$. Let $\left(\sigma_{\xi} \mid \xi<\omega_{1}\right)$ be a continuous, exhaustive chain in $[\gamma]^{\omega}$ and let $\bar{A}=\left\{\xi \mid \sigma_{\xi} \in A\right\}$ and similarly define $\bar{B}$. Let $G_{A}, G_{B} \subset P\left(\omega_{1}\right) / N S$ be $V$-generic with $\bar{A} \in G_{A}$ and $\bar{B} \in G_{B}$. Let $g \subset \operatorname{Col}(\omega, \gamma)$ be generic over both $V\left[G_{A}\right]$ and $V\left[G_{B}\right]$ with $r \in g$. Thus

$$
J_{\alpha}(\mathbb{R})^{V\left[G_{A}\right][g]}=\psi\left(F \left(\left(\tau_{1}\lceil\gamma)_{g}\right), \ldots, F\left(\left(\tau_{k}\lceil\gamma)_{g}\right)\right)\right.\right.
$$

and

$$
J_{\alpha}(\mathbb{R})^{V\left[G_{B}\right][g]} \models \phi\left(F \left(\left(\tau_{1}\lceil\gamma)_{g}\right), \ldots, F\left(\left(\tau_{k}\lceil\gamma)_{g}\right)\right) .\right.\right.
$$

By hypothesis there are $\Sigma_{1}$ embeddings

$$
\bar{\pi}_{A}: J_{\alpha}(\mathbb{R})^{V[g]} \rightarrow J_{\alpha}(\mathbb{R})^{V\left[G_{A}\right][g]}
$$

and

$$
\bar{\pi}_{B}: J_{\alpha}(\mathbb{R})^{V[g]} \rightarrow J_{\alpha}(\mathbb{R})^{V\left[G_{B}\right][g]} .
$$

Thus $J_{\alpha}(\mathbb{R})^{V[g]}$ satisfies

$$
\psi\left(F \left(\left(\tau_{1}\lceil\gamma)_{g}\right), \ldots, F\left(\left(\tau_{k}\lceil\gamma)_{g}\right)\right) \wedge \neg \psi\left(F \left(\left(\tau_{1}\lceil\gamma)_{g}\right), \ldots, F\left(\left(\tau_{k}\lceil\gamma)_{g}\right)\right),\right.\right.\right.\right.
$$

which is the desired contradiction. The other direction of the negation case follows similarly. 
We now treat the unbounded existential case. For the nontrivial direction suppose for a club of $\sigma \in\left[\omega_{2}\right]^{\omega}$ that

$$
p^{\sigma} \Vdash_{C o l(\omega, o t p(\sigma))} J_{l\left(\sigma \cap \omega_{1}\right)}(\mathbb{R}) \models \exists x \phi\left(x, F\left(\tau^{\sigma}\right)\right) .
$$

For simplicity we assume there is only one parameter. For a real $z$, the set

$$
\{x \mid \phi(F(x), F(z))\}
$$

as interpreted in a level of $L(\mathbb{R})$ beginning a gap, is the projection of the tree of the $\Sigma_{1}$-scale on this set. We let $l w(z)$ denote witness obtained from the leftmost branch of this tree. A key point is that there is a $\Sigma_{1}$ formula $\psi$ so that $\psi(u, z)$ holds if and only if $u=l w(z)$. We define a term $l w(\tau)$ as follows. For a condition $q$ and a pair $n, m \in \omega$ we put the term $(q,(n, m))$ (abusing notation) in $l w(\tau)$ if and only if for a club of $\sigma$,

$$
q^{\sigma} \Vdash_{C o l(\omega, o t p(\sigma))} J_{l\left(\sigma \cap \omega_{1}\right)}(\mathbb{R}) \models l w\left(\tau^{\sigma}\right)(n)=m .
$$

We need to see that for a club of $\sigma \in\left[\omega_{2}\right]^{\omega}$

$$
p^{\sigma} \Vdash_{C o l(\omega, o t p(\sigma))} J_{l\left(\sigma \cap \omega_{1}\right)}(\mathbb{R}) \models l w(\tau)^{\sigma}=l w\left(\tau^{\sigma}\right) .
$$

We will then have

$$
p^{\sigma} \Vdash_{C o l(\omega, o t p(\sigma))} J_{l\left(\sigma \cap \omega_{1}\right)}(\mathbb{R}) \models \phi\left(F\left(\left(l w(\tau)^{\sigma}\right), F\left(\tau^{\sigma}\right)\right)\right.
$$

for each such $\sigma$ as desired. Assume otherwise. We extract a condition $q$ below $p$, integers $n, m_{1}, m_{2}$, an ordinal $\gamma<\omega_{2}$ above $q$ and stationary sets $A, B \subset[\gamma]^{\omega}$ such that for $\sigma \in A$ we have

$$
q^{\sigma} \Vdash_{C o l(\omega, o t p(\sigma))} J_{l\left(\sigma \cap \omega_{1}\right)}(\mathbb{R}) \models l w\left(\tau^{\sigma}\right)(n)=m_{1}
$$

and for $\sigma \in B$ we have

$$
q^{\sigma} \Vdash_{C o l(\omega, o t p(\sigma))} J_{l\left(\sigma \cap \omega_{1}\right)}(\mathbb{R}) \models l w\left(\tau^{\sigma}\right)(n)=m_{2} .
$$

As in the negation case we get generics $G_{A}, G_{B} \subset P\left(\omega_{1}\right) / N S$ and $g \subset \operatorname{Col}(\omega, \gamma)$ such that

$$
J_{\alpha}(\mathbb{R})^{V\left[G_{A}\right][g]} \models l w\left((\tau \uparrow \gamma)_{g}\right)(n)=m_{1}
$$

and

$$
J_{\alpha}(\mathbb{R})^{V\left[G_{B}\right][g]} \models l w\left((\tau \uparrow \gamma)_{g}\right)(n)=m_{2} .
$$

Using $\bar{\pi}$ we get a contradiction. This completes the proof of the claim. 
Now let $h \subset \operatorname{Col}\left(\omega, \omega_{2}\right)$ be $V$-generic. In $V[h]$ we form a structure

$$
(M, E)=\left(\dot{M}_{h} / \dot{=}_{h}, \dot{\epsilon}_{h} / \dot{=}_{h}\right) \text {. }
$$

An easy argument which uses the fact that the club filter on $P\left(\left[\omega_{2}\right]^{\omega}\right)$ is countably complete shows that $M$ is wellfounded, and so there is an isomorphism

$$
i:(M, E) \rightarrow(N, \in)
$$

for some transitive set $N$. The construction of the function $F$ we have used is such that for any real $r$ there is a real $\hat{r}$ obtained from $r$ so that $F(\hat{r})=r$ and $r, \hat{r}$ are Turing equivalent. Thus for any standard term for a real $\tau$, there is a term $\hat{\tau}$ so that

$$
\hat{\tau}_{h}=\hat{\tau_{h}} .
$$

It follows that $\mathbb{R}^{N}=\mathbb{R}^{V[h]}$. By claim 45, $N$ satisfies the sentence asserting that it is a level of $L(\mathbb{R})$ and thus $N=J_{\alpha^{*}}(\mathbb{R})$ in $V[h]$, for some ordinal $\alpha^{*}$ (which would seem to depend on $h$ ). We finish the proof of lemma 44 by showing that the following are equivalent for a $\Sigma_{1}$ formula $\phi$, a condition $p \in \operatorname{Col}\left(\omega, \omega_{2}\right)$ and a standard term for a real $\tau$.

(1) $p \Vdash \dot{\mathcal{M}}=\phi(\tau)$

(2) $p \Vdash J_{\alpha^{*}}(\mathbb{R}) \models \phi(F(\tau))$

Note that $\dot{M} \models F(\hat{\tau})=\tau$ so $J_{\alpha}^{*}(\mathbb{R}) \models F(i(\hat{\tau})=i(\tau)$. Since $i(\hat{\tau})=\tau$ we see that $i=F$, which gives the equivalence above. The equivalence shows that $J_{\alpha^{*}}(\mathbb{R})^{V[h]}$ is $\Sigma_{1}$-generated by its reals, so that $\alpha^{*}$ begins a gap in $V[h]$.

If $h$ is $\operatorname{Col}\left(\omega, \omega_{2}\right)$-generic over $V$, then we write $\alpha^{*}(h)$ for the ordinal given by lemma 44. We shall eventually show $\alpha^{*}(h)$ is independent of $h$.

Lemma 46. Let $g$ be $\operatorname{Col}\left(\omega, \omega_{1}\right)$-generic over $V$, and suppose that $g \in V[h]$, where $h$ is $\operatorname{Col}\left(\omega, \omega_{2}\right)$-generic over $V$; then there is a $\Sigma_{2}$ elementary embedding

$$
\pi_{2}: J_{\alpha}(\mathbb{R})^{V[g]} \rightarrow J_{\alpha^{*}(h)}(\mathbb{R})^{V[h]} .
$$

Proof. We consider first the case that $h=g \times k$, where $k$ is $\operatorname{Col}\left(\omega, \omega_{2}^{V}\right)$ generic over $V[g]$. The proof of 44 generalizes trivially from $\operatorname{Col}\left(\omega, \omega_{2}\right)$ to $\operatorname{Col}\left(\omega, \omega_{1}\right) \times \operatorname{Col}\left(\omega, \omega_{2}\right)$, and shows that the ordinal

$$
\alpha^{*}=\alpha^{*}(h)=\alpha^{*}(g \times k)
$$


that begins a gap in $V[g][k]$ and satisfies the natural variant of the Los property recorded in the statement of 44 . We show that $J_{\alpha}(\mathbb{R})^{V[g]}$ $\Sigma_{2}$-embeds into $J_{\alpha^{*}}(\mathbb{R})^{V[g][k]}$.

Since $J_{\alpha}(\mathbb{R})^{V[g]}$ is pointwise $\Sigma_{1}$-definable from its reals, it is enough to show that whenever $x$ is a real in $V[g]$ and $\varphi(v)$ is a $\Sigma_{2}$ formula, then

$$
J_{\alpha}(\mathbb{R})^{V[g]}=\varphi[x] \Leftrightarrow J_{\alpha^{*}}(\mathbb{R})^{V[g][k]}=\varphi[x] .
$$

We show this first for $\varphi(v)$ a $\Sigma_{1}$ formula. Suppose $J_{\alpha^{*}}(\mathbb{R})^{V[g][k]}=$ $\varphi[x]$. Let $x=\rho^{g}$, where $\rho$ is a standard $\operatorname{Col}\left(\omega, \omega_{1}\right)$ term for a real. Let

$$
(p, q) \Vdash J_{\alpha^{*}(\dot{g} \times \dot{k})}(\mathbb{R}) \models \varphi\left(\rho^{\dot{g}}\right),
$$

with $(p, q) \in g \times k$. Applying 44, we have a club $C$ of $\sigma \in\left[\omega_{1} \times \omega_{2}\right]^{\omega}$ such that, letting $\sigma_{0}=\{\xi \mid \exists \nu\langle\xi, \nu\rangle \in \sigma\}$ and $\sigma_{1}=\{\nu \mid \exists \xi\langle\xi, \nu\rangle \in \sigma\}$, we have

$$
\left(p, q^{\sigma}\right) \Vdash J_{l\left(\sigma_{0} \cap \omega_{1}\right)}(\mathbb{R}) \models \varphi\left[\left(\rho^{\sigma_{0}}\right)^{\dot{g}}\right],
$$

where the forcing is $\operatorname{Col}\left(\omega, \sigma_{0} \cap \omega_{1}\right) \times \operatorname{Col}\left(\omega, o t p\left(\sigma_{1}\right)\right)$. Thus the forcing is essentially Cohen forcing. Note that $\sigma_{0}$ is transitive, $p^{\sigma_{0}}=p$, and $\rho^{\sigma_{0}}=\rho \cap \sigma_{0}$, for club many $\sigma$. We have by $\operatorname{WRP}_{(2)}\left(\omega_{2}\right)$ a $\gamma<\omega_{2}$ such that $C$ is club in $\left[\omega_{1} \times \gamma\right]^{\omega}$, and $q \in \gamma^{<\omega}$. Now let $G$ be such that $g \times G$ is $\operatorname{Col}\left(\omega, \omega_{1}\right) \times P\left(\omega_{1}\right) / N S$-generic over $V$. (It doesn't matter whether $G \in V[h]$.) Los theorem for the generic ultrapower by $G$ gives

$$
V[G] \models(p, q) \Vdash J_{\alpha}(\mathbb{R}) \models \varphi\left[\rho^{\dot{g}}\right],
$$

which gives

$$
V[G][g] \models q \Vdash J_{\alpha}(\mathbb{R}) \models \varphi[x],
$$

where the forcing is $\operatorname{Col}(\omega, \gamma)$, and hence essentially Cohen forcing. Since $\sum_{1}^{J_{\alpha}(\mathbb{R})}$ has the Baire property in $V[G][g]$, we get that

$$
J_{\alpha}(\mathbb{R})^{V[G][g]}=\varphi[x],
$$

and hence

$$
J_{\alpha}(\mathbb{R})^{V[g]} \models \varphi[x],
$$

as desired.

The proof just given works equally well for $\Pi_{1}$ formulae $\varphi$, and so it gives the desired equivalence for $\Sigma_{1}$ formulae. That in turn gives us a unique $\Sigma_{1}$ elementary embedding $\pi_{2}: J_{\alpha}(\mathbb{R})^{V[g]} \rightarrow J_{\alpha^{*}}(\mathbb{R})^{V[g][k]}$. We want to show $\pi_{2}$ is $\Sigma_{2}$-elementary. For that, it suffices to show that $\Sigma_{2}$ formulae about a real $x$ go down, and since $\alpha^{*}$ begins a gap in $V[g][k]$, we may assume the outer quantifier is witnessed by a real. Let $x=\rho^{g}=\mu^{g \times k}$. We have

$$
J_{\alpha^{*}}(\mathbb{R})^{V[g][k]}=\varphi\left[\rho^{g}, \nu^{g \times k}\right],
$$


where $\nu$ is a canonical term for a real, and $\varphi(v)$ is $\Pi_{1}$. Let $(p, q)$ force this. As before, we get a $\gamma<\omega_{2}$ such that for club many $\sigma \in\left[\omega_{1} \times \gamma\right]^{\omega}$,

$$
\left(p, q^{\sigma}\right) \Vdash J_{l\left(\sigma_{0} \cap \omega_{1}\right)}(\mathbb{R}) \models \varphi\left[\left(\rho^{\sigma_{0}}\right)^{\dot{g}},\left(\nu^{\sigma}\right)^{\dot{g} \times \dot{k}}\right] .
$$

Again, let $G$ be such that $g \times G$ is $\operatorname{Col}\left(\omega, \omega_{1}\right) \times P\left(\omega_{1}\right) / N S$ generic over $V$. We have

$$
V[G] \models(p, q) \Vdash J_{\alpha}(\mathbb{R}) \models \varphi\left[\rho^{\dot{g}}, \nu^{\dot{g} \times \dot{k}}\right],
$$

which gives

$$
V[G][g] \models q \Vdash J_{\alpha}(\mathbb{R}) \models \varphi[x, \hat{\nu}],
$$

where $\hat{\nu}$ is a $\operatorname{Col}(\omega, \gamma)$ term obtained from $\nu$ and $g$. Let $k_{0}$ be $\operatorname{Col}(\omega, \gamma)$ generic over $V[G][g]$, and let $z=\nu^{g \times k_{0}}$. We have

$$
V[G][g]\left[k_{0}\right] \models J_{\alpha}(\mathbb{R}) \models \varphi[x, z] .
$$

But then

$$
V[g]\left[k_{0}\right] \models J_{\alpha}(\mathbb{R}) \models \varphi[x, z]
$$

because $g \times k_{0}$ is equivalent to a $\operatorname{Col}\left(\omega, \omega_{1}\right)$ generic over $V$, and we have $I_{\alpha}$. Since $\sum_{1}^{J_{\alpha}(\mathbb{R})}$ has the Baire property in $V[g], 38$ gives

$$
V[g] \models J_{\alpha}(\mathbb{R}) \models \exists z \varphi[x, z],
$$

as desired.

Thus we have a unique $\Sigma_{2}$ elementary

$$
\pi_{2}: J_{\alpha}(\mathbb{R})^{V[g]} \rightarrow J_{\alpha^{*}(g \times k)}(\mathbb{R})^{V[g][k]} .
$$

Let $\varphi, x$ be a witness to the inadmissibility of $\alpha$ in $V[g]$; that is, $\varphi(u, v)$ is $\Sigma_{1}, x \in \mathbb{R} \cap V[g]$, and $\alpha$ is the least ordinal $\beta$ such that $J_{\beta}(\mathbb{R})^{V[g]}=$ $\forall u \in \mathbb{R} \varphi[x]$. Since $\pi_{2}$ is $\Sigma_{2}$ elementary, $\varphi, x$ witness the inadmissibility of $\alpha^{*}(g \times k)$ in $V[g][k]$, so that $\varphi, x$ gives us a "name" for $\alpha^{*}(g \times k)$.

We now show $\alpha^{*}(h)$ is independent of $h$. It is enough to show $\alpha^{*}\left(h_{1}\right)=\alpha^{*}\left(h_{2}\right)$ whenever $h_{1}$ and $h_{2}$ are $\operatorname{Col}\left(\omega, \omega_{2}\right)$-generic, and $V\left[h_{1}\right]=$ $V\left[h_{2}\right]$. We may re-arrange $h_{i}$ so that $h_{i}=g_{i} \times k_{i}$, where $g_{i} \times k_{i}$ is $\operatorname{Col}\left(\omega, \omega_{1}\right) \times \operatorname{Col}\left(\omega, \omega_{2}\right)$-generic. Let $\varphi_{i}, x_{i}$ be an inadmissibility witness for $\alpha$ in $V\left[g_{i}\right]$. Since $\Sigma_{1}^{J_{\alpha}(\mathbb{R})}$ has the Baire property in $V\left[g_{i}\right]$, we have that in $V\left[g_{1}\right]\left[g_{2}\right]$, both $\varphi_{1}, x_{1}$ and $\varphi_{2}, x_{2}$ are inadmissibility witnesses for $\alpha$. Let $g_{3}=g_{1} \times g_{2}$, and let $k_{3}$ on $\operatorname{Col}\left(\omega, \omega_{2}\right)$ be such that $V\left[g_{3}\right]\left[k_{3}\right]=V\left[h_{1}\right]=V\left[h_{2}\right]$. By what we just showed, both $\varphi_{1}, x_{1}$ and $\varphi_{2}, x_{2}$ are inadmissibility witnesses for $\alpha^{*}\left(g_{3} \times k_{3}\right)$ in $V\left[h_{1}\right]=V\left[h_{2}\right]$. It follows that $\alpha^{*}\left(h_{1}\right)=\alpha^{*}\left(g_{3} \times k_{3}\right)=\alpha^{*}\left(h_{2}\right)$, as desired.

This finishes the proof of lemma 46.

Henceforth we write $\alpha^{*}$ for the common value of all $\alpha^{*}(h)$. 
Lemma 47. Let $g \times G$ be $\operatorname{Col}\left(\omega, \omega_{1}\right) \times P\left(\omega_{1}\right) / N S$ generic over $V$, and suppose $g, G \in V[h]$, where $h$ is $\operatorname{Col}\left(\omega, \omega_{2}\right)$ generic over $V$; then there is a unique $\Sigma_{1}$ elementary

$$
j: J_{\alpha}(\mathbb{R})^{V[G][g]} \rightarrow J_{\alpha^{*}}(\mathbb{R})^{V[h]} .
$$

Moreover, $\pi_{2}=j \circ \pi$.

Proof. In $V[h], J_{\alpha^{*}}(\mathbb{R}) \models \mathrm{AD}$, and $\alpha^{*}$ begins a gap. Moreover, $V[h]=V[k]$ where $k$ is $\operatorname{Col}\left(\omega, \omega_{2}^{V}\right)$ generic over $V[G][g]$. Thus by lemma 36, there is a unique $\gamma$ beginning a gap in $V[G][g]$ and a unique $\Sigma_{1}$-elementary

$$
j: J_{\gamma}(\mathbb{R})^{V[G][g]} \rightarrow J_{\alpha^{*}}(\mathbb{R})^{V[h]} .
$$

It will be enough to show $\gamma=\alpha$. (We then get $\pi_{2}=j \circ \pi$ because the maps are determined by their restrictions to reals.)

To see $\gamma=\alpha$, in $V[g]$ let $B$ be the prewellordering associated to a $\Sigma_{1}^{J_{\alpha}(\mathbb{R})}$ norm on a universal $\Sigma_{1}^{J_{\alpha}(\mathbb{R})}$ set of reals. $B$ has length $\alpha$. In $V[h]$, let $C$ be the set with the same definition, but over $J_{\alpha^{*}}(\mathbb{R})$. Since $\Sigma_{1}^{J_{\alpha^{*}}(\mathbb{R})}$ has the scale property in $V[h]$, there are definable-in- $V[h]$ trees $T, U$ projecting to $C$ and its complement. By homogeniety, $T$ and $U$ are in $V$. Because $\pi_{2}$ exists, $T$ and $U$ project to $B$ and its complement in $V[h]$. It follows that

$$
V[g] \models B \text { is } \omega_{2} \text {-universally Baire. }
$$

But $G$ is generic over $V[h]$ for a reasonable forcing, so by the ForemanMagidor theorem 34, every level of $C$ with a representative in $V[G][g]$ has a representative in $V[g]$. The order type of $C \cap V[G][g]$ is just $\gamma$, and the order type of $B=C \cap V[g]$ is $\alpha$, so $\gamma=\alpha$.

We now consider the case that $\alpha$ is in the range of a generic embedding.

Lemma 48. Suppose $\pi_{G}\left(\alpha_{0}\right)=\alpha$ for some $P\left(\omega_{1}\right) / N S$ generic; then

(1) $J_{\alpha_{0}}(\mathbb{R})$ is inadmissible in $V$, and

(2) $\pi_{G}\left(\alpha_{0}\right)=\alpha$ for all $P\left(\omega_{1}\right) / N S$ generic $G$.

Proof. Let $g$ be such that $g \times G$ is $\operatorname{Col}\left(\omega_{1}, \omega\right) \times P\left(\omega_{1}\right) / N S$ generic. Let $h$ on $\operatorname{Col}\left(\omega, \omega_{2}\right)$ be such that $g, G \in V[h]$. In $V[g]$, let $x$ be a real and $\varphi$ a $\Sigma_{1}$ formula such that

$$
J_{\alpha}(\mathbb{R}) \models \forall y \in \mathbb{R} \varphi[y, x],
$$

and $\alpha$ is least such that this is true. So $\varphi, x$ witness the inadmissibility of $\alpha$ in $V[g]$. Because $\pi_{2}$ is $\Sigma_{2}$ elementary, $\varphi$ and $x$ witness the inadmissiblity of $\alpha^{*}$ in $V[h]$. But then our $\Sigma_{1}$ map $j$ from 47 shows that $\varphi, x$ define a total function over $J_{\alpha}(\mathbb{R})$ in $V[G][g]$, and the map $\pi$ given 
by $I_{\alpha}$ shows this function has range cofinal in $\alpha$. Thus $\varphi, x$ witness the inadmissibilty of $\alpha$ in $V[G][g]$. The Cohen map is $\Sigma_{2}$, so $\varphi, x$ witness the inadmissibility of $\alpha$ in $V[G]$. Finally, $\pi_{G}$ is fully elementary, and this gives us part (1).

For (2), let $\varphi, x$ witness the inadmissibility of $\alpha_{0}$ in $V$. Let $g \times G$ be any $\operatorname{Col}\left(\omega_{1}, \omega\right) \times P\left(\omega_{1}\right) / N S$ generic, with $\alpha=\pi_{G}\left(\alpha_{0}\right)$. Then $\varphi, x$ witnesses inadmissibilty of $\alpha$ in $V[G]$, hence $V[G][g]$, and hence $V[g]$. If $k \times H$ is any other generic, and $\xi=\pi_{H}\left(\alpha_{0}\right)$, then $\varphi, x$ witness inadmissibility for $\xi$ in $V[k]$. Since $x \in V$ and $\operatorname{Col}\left(\omega, \omega_{1}\right)$ is homogeneous, $\xi=\alpha$, as desired.

Lemma 49. Suppose $\alpha=\pi_{G}\left(\alpha_{0}\right)$ for some $G$; then $I_{\alpha+1}$ holds, and $W_{\alpha+1}^{*}$ holds in all $V[g]$, for $g$ on $\operatorname{Col}\left(\omega, \omega_{1}\right)$.

Proof. Let $\varphi, x$ witness the inadmissibility of $\alpha_{0}$ in $V$. Let $g \times G$ be $\operatorname{Col}\left(\omega_{1}, \omega\right) \times P\left(\omega_{1}\right) / N S$ generic. Let $h$ on $\operatorname{Col}\left(\omega, \omega_{2}\right)$ be such that $g, G \in V[h]$. We have that $\varphi, x$ witnesses inadmissibility for $\alpha$ in $V[g]$, and for $\alpha^{*}$ in $V[h]$. Working in $V[h]$, we now obtain a function $J$ on $H\left(\omega_{1}\right)$ with parameter $x$ such that $J$ codes up $\Sigma_{1}^{J_{\alpha^{*}}(\mathbb{R})}$ truth. Here we just follow the construction of [22]. Given a countable transitive set $b$ such that $x \in b, J(b)$ is the minimal $b$-premouse with parameter $x$, call it $\mathcal{M}$, such that

$$
J_{\alpha^{*}}(\mathbb{R}) \models \mathcal{M} \text { is } \omega_{1} \text {-iterable },
$$

and

$$
\mathcal{M} \models \psi \text {. }
$$

Here $\psi$ asserts roughly that the $\Sigma_{1}$ function defined by $\varphi, x$ is defined at all reals of the form $\tau_{g}$, where $g$ is $\operatorname{Col}(\omega, b)$ generic over $\mathcal{M}$ and $\tau$ is a term that is rudimentary in $b$.

More precisely, if $l \subset \operatorname{Col}(\omega, b)$ is $\mathcal{M}$-generic, then there is a real $z(l, b)$ which simply codes the pair $(l, b)$. Thus $\mathcal{M}[l]$ is a $z(l, b)$-mouse. Let $\sigma \in \mathcal{M}$ be term so that whenever $l$ is such a generic

$$
\left\{\left(\sigma_{l}\right)_{i} \mid i>0\right\}=\left\{\rho_{l} \mid \rho \in L_{1}(b)\right\}
$$

and $\left(\sigma_{l}\right)_{0}=x$. We let the sentence $\psi$ assert that whenever $l$ is generic there is a $\gamma$ so that $\mathcal{M}(z(l, b)) \uparrow \gamma$ is a $\left(\phi_{n}^{*}, \sigma_{l}\right)$-witness, in the sense referred to in $W^{*}$, where $\phi_{n}^{*}(v)$ is the $\Sigma_{1}$ formula: there is an ordinal $\xi$ for which $\omega \xi+n$ exists and

$$
J_{\xi}(\mathbb{R}) \models \forall i>0 \phi\left(v_{i}, v_{0}\right) .
$$

Since in $V[h], \varphi, x$ witnesses inadmissibliity for $J \alpha^{*}(\mathbb{R})$, and $J_{\alpha^{*}}(\mathbb{R})$ thinks that every $\Sigma_{1}$ truth about a real is captured by an $\omega_{1}$-iterable 
premouse, we have that $J(b)$ exists for all $b$ such that $x \in b . J$ is not quite a mouse operator in $V[h]$, because that requires $\omega_{1}+1$-iterability. However, $\omega_{1}$-iterability in a model of $A D$ is enough for comparison, so $J(b)$ is $\Sigma_{1}$-definable over $J_{\alpha^{*}}(\mathbb{R})$ from Letting $J_{0}=J \uparrow V$, we then have in $V$ that $J_{0}$ is a first order mouse operator on $H\left(\omega_{3}\right)$, and $J=J_{0}^{h}$. It is not hard to see that $J_{0}$ relativises well, condenses well, and determines itself on generic extensions. (See [22].)

Our lemmas 47 on the existence of $j$ and the Cohen ultrapower map $\psi$ imply that $J$ is NS-absolute. For let $k \times H$ be $\operatorname{Col}\left(\omega, \omega_{1}\right) \times P\left(\omega_{1}\right) / N S$ generic over $V$, and both in $V[l]$, where $l$ is $\operatorname{Col}\left(\omega, \omega_{2}\right)$ generic. Let $\theta$ be the $\Sigma_{1}$ formula defining $J_{0} \uparrow H\left(\omega_{1}\right)^{V}$ from $x$ over $J_{\alpha_{0}}(\mathbb{R})$. Then $\theta$ defines $J^{l}$ over $J_{\alpha^{*}}(\mathbb{R})^{V[l]}$, because $j \circ \psi \circ \pi_{H}$ exists. Thus $\theta$ defines $J^{l} \uparrow V[H]=J^{H}$ over $J_{\alpha}(\mathbb{R})^{V[H]}$, because $j \circ \psi$ exists. So $\pi_{H}\left(J_{0}\right) \subseteq J^{H}$.

So in $V, J_{0}$ is an $\left(\omega_{3}, x\right)$ mouse operator that relativises and condenses well, determines itself on generic extensions, and is NS-absolute. By theorem 28, setting

$$
J_{n+1}=\left(J_{n}\right)^{w},
$$

we have the same properties for $J_{n}$, for all $n<\omega$.

This gives $W_{\alpha_{0}+1}^{*}$ in $V$, with the witnessing mice being the $J_{n}(b)$. Going back to $g, G$, and $h$, we also have that $W_{\alpha+1}^{*}$ holds in $V[g]$, with the witnessing mice being those of the form $J_{n}^{g}(b)$ for $b \in H\left(\omega_{1}\right)^{V[g]}$. For details on why these mice suffice as witnesses, see [22]; the key is that $\Sigma_{n}$ truth over $J_{\alpha}(\mathbb{R})$ reduces to $\left(\Sigma_{n}^{1}\right)$-in- $J_{0}$ truth by inadmissibility.

We also get $I_{\alpha+1}$. For let

$$
\pi: J_{\alpha}(\mathbb{R})^{V[g]} \rightarrow J_{\alpha}(\mathbb{R})^{V[G][g]}
$$

be the $\Sigma_{1}$ map given by $I_{\alpha}$. It suffices to show $\pi$ is fully elementary. This is because the $J_{n}$ 's code truth over $J_{\alpha}(\mathbb{R})$. More precisely, fix $n<\omega$. There is a recursive function $t$ such that for any $\Sigma_{n}$ formula $\varphi$ and any $b$ in the $H\left(\omega_{1}\right)$-cone over $x$ of $V$, we have

$$
J_{\alpha_{0}}(\mathbb{R})^{V} \models \varphi[b, x] \Leftrightarrow J_{n}(b) \models t(\varphi)[b] .
$$

But then for any $b \in H\left(\omega_{1}\right)^{V[G]}$,

$$
J_{\alpha}(\mathbb{R})^{V[G]} \models \varphi[b, x] \Leftrightarrow \pi_{G}\left(J_{n}\right)(b) \models t(\varphi)[b] .
$$

Since $J_{\alpha+1}(\mathbb{R})^{V[G]} \models \mathrm{AD}$, the Cohen ultrapower map $\psi$ is fully elemen-

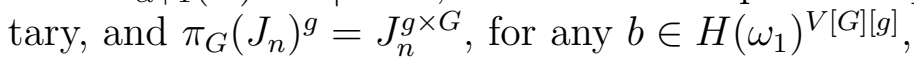

$$
J_{\alpha}(\mathbb{R})^{V[G][g]}=\varphi[b, x] \Leftrightarrow J_{n}^{g \times G}(b) \models t(\varphi)[b] .
$$

Finally, for $b \in H\left(\omega_{1}\right)^{V[g]}$,

$$
J_{\alpha}(\mathbb{R})^{V[g]} \models \varphi[b, x] \Leftrightarrow J_{n}^{g} \models t(\varphi)[b] .
$$


Since $J_{n}^{g} \subseteq J_{n}^{g \times G}$, this gives us that the inclusion map on the reals is fully elementary from $J_{\alpha}(\mathbb{R})^{V[g]}$ to $J_{\alpha}(\mathbb{R})^{V[G][g]}$. That implies $\pi$ is fully elementary, and hence $I_{\alpha+1}$.

We will be done with the inadmissible, uncountable cofinality case when we show:

Lemma 50. Suppose $\alpha<\pi_{G}\left(\alpha_{0}\right)$ for some $G$; then $I_{\alpha+1}$ holds, and $W_{\alpha+1}^{*}$ holds in all $V[g]$, for $g$ on $\operatorname{Col}\left(\omega, \omega_{1}\right)$.

Proof. Let $g \times G$ be $\operatorname{Col}\left(\omega_{1}, \omega\right) \times P\left(\omega_{1}\right) / N S$ generic. Let $h$ on $\operatorname{Col}\left(\omega, \omega_{2}\right)$ be such that $g, G \in V[h]$. Let $\pi$ be given by $I_{\alpha}, \pi_{2}$ by lemma $46, \psi$ by the Cohen ultrapower, and $j$ by lemma 47. We have that $\pi$ and $j$ are $\Sigma_{1}$ elementary, while $\psi$ and $\pi_{2}$ are $\Sigma_{2}$ elementary. Let $\varphi, x$ witnesses inadmissibility for $\alpha$ in $V[g], V[G]$, and $V[G][g]$, and for $\alpha^{*}$ in $V[h]$.

Just as before, working in $V[h]$, we obtain a function $J_{0}$ on $H\left(\omega_{1}\right)$ with parameter $x$ such that $J_{0}$ codes up $\Sigma_{1}^{J_{\alpha^{*}}(\mathbb{R})}$ truth. $J_{0}(b)$ is the least $b$-premouse with parameter $x$ satisfying a certain sentence $\varphi$, and being $\omega_{1}$-iterable in $J_{\alpha^{*}}(\mathbb{R})$. The difference now is just that the parameter $x$ may only be in $V[g]$. Since $V[h]$ is a homogeneous extension of $V[g]$, we do have in $V[g]$ and $V[G][g]\left(\omega_{2}, x\right)$ mouse operators $K_{0}, K_{1}$ such that $J_{0}=K_{i}^{h} . K_{i}$ is just the restriction of $J_{0}$ to the $H\left(\omega_{2}\right)$ of its model, so we write $J_{0}$ for $K_{i}$.

Claim. In $V[h]$, there are functions $J_{n}$ on the $H\left(\omega_{1}\right)$ cone over $x$ such that for any $b$ in this cone

(a) $J_{n+1}(b) \models$ "I am $J_{n}^{w}(b)$ ", and

(b) $J_{n+1}(b)$ is $\omega_{1}$ iterable in $V[h]$ by a $J_{n}$-guided iteration strategy.

Remark. So $J_{n+1}$ is $J_{n}^{w}$, except that $\omega_{1}+1$ strategies have to be replaced in $V[h]$ by absolutely definable $\omega_{1}$ strategies.

Proof.

We begin with $J_{1}$, though our method works in general.

Subclaim 1. In $V[g]$, for any $b$ in the $H\left(\omega_{1}\right)$ cone over $x, J_{0}^{w}(b)$ exists, and has a $J_{0}$-guided $\omega_{2}$-iteration strategy.

Proof. Fix $b$. We have $b$ in $V[G][g]$. Because $j$ exists, $J_{0} \uparrow V[G][g]$ has the same definition over $J_{\alpha}(\mathbb{R})$ in $V[G][g]$ that $J_{0}$ has over $J_{\alpha^{*}}(\mathbb{R})$ in $V[h]$. But $\pi_{G}\left(\alpha_{0}\right)>\alpha$, and hence

$$
V[G] \models W_{\alpha+1}^{*},
$$

so that

$$
V[G][g] \models W_{\alpha+1}^{*},
$$


by the elementarity of the Cohen ultrapower. Hence in $V[G][g]$ we can find $\mathcal{M}$ such that

(a) $\mathcal{M} \models$ "I am $J_{0}^{w}(b) "$, and

(b) $V[G][g] \models$ " $\mathcal{M}$ has a $J_{0}$-guided $\omega_{1}$-iteration strategy".

Using condensation for $J_{0}$ in $V[G][g]$ and the fact that it determines itself on generic extensions, we see by a standard Lowenheim-Skolem argument that $\mathcal{M}$ has a $J_{0}$-guided $\omega_{1}$-iteration strategy in $V[h]$. This fact defines $\mathcal{M}$ in $V[h]$ from $J_{0} \uparrow V[g]$ and $b$, so $\mathcal{M} \in V[g]$. It is easy to see that $V[g] \models \mathcal{M}=J_{0}^{w}(b)$, and that $\mathcal{M} J_{0}$-guided $\omega_{2}$-iteration strategy in $V[g]$.

Subclaim 2. In $V[h]$, for any $b$ in the $H\left(\omega_{1}\right)$ cone over $x$, there is a $b$ premouse $\mathcal{M}$ with parameter $x$ such that

(a) $\mathcal{M} \models$ "I am $J_{0}^{w}(b) "$, and

(b) $V[h] \models$ "M has a $J_{0}$-guided $\omega_{1}$-iteration strategy".

Proof. We go back to $V$, so that we can use Mouse Reflection.

Let $\tau$ be a standard term such that $\tau_{g}=x$. Thus $\tau \in V$, and $\tau \subset \omega \times \omega_{1}$. Working in $V$, we can find for any $b$ in the $H\left(\omega_{2}\right)$ cone over $\tau$ a countably iterable $b$-premouse $\mathcal{M}$ with parameter $\tau$ such that $J_{0}^{w}(b)$ is the canonical re-arrangement of $\mathcal{M}[g]$ as a premouse over $\langle b, g\rangle$ with parameter $x$. (The hierarchy of $\mathcal{M}$ is defined by induction. See [24] or [14] for the details of this method of inverting generic extensions of mice.) Writing $\mathcal{M}=I(b)$, we can summarize this as

$$
I(b)[g]=J_{0}^{w}(b),
$$

for all $b$ in the $H\left(\omega_{2}\right)^{V}$-cone over $\tau$. $I$ is a first order mouse operator with parameter $\langle\tau, p\rangle$ in $V$, its sentence being "it is forced in $\operatorname{Col}\left(\omega, \omega_{1}\right)$ by $p$ that my canonical re-arrangement as a premouse over $\langle b, g\rangle$ with parameter $\tau_{g}$ thinks it is $J_{0}^{w}(b)$." (Here $p$ is an appropriately chosen fixed condition.)

By Mouse Reflection, $I$ extends in $V$ to an $\left(\omega_{3},\langle p, \tau\rangle\right)$ mouse operator, which we also call $I$. Given now $b$ in the $H\left(\omega_{1}\right)^{V[h]}$ cone over $x$, we can find an $\mathcal{M}$ as in subclaim 2 as follows. Let $V[h]=V[g][k]$, where $k$ is $\operatorname{Col}\left(\omega, \omega_{2}\right)$ generic over $V[g]$, and let $b=\sigma_{g}$. Let $c$ be in the domain of $I$, with $\tau, \sigma \in c$. Then $I(c)[g][k]$ can be re-arranged as a premouse over $\langle c, g, k\rangle$ with parameter $x=\tau_{g}$. Let $\mathcal{N}$ be this re-arrangement. It is easy to see

(a) $\mathcal{N} \models$ "I am $J_{0}^{w}(\langle c, g, k\rangle)$ ", and

(b) $V[g][k] \models$ "N has a $J_{0}$-guided $\omega_{1}$-iteration strategy". 
The reason is simply that we can reflect any failure of (a) or (b) into the $H\left(\omega_{1}\right)$ cone over $x$ of $V[g]$, where $I$ does indeed determine $J_{0}^{w}$ by the method whereby we obtained $\mathcal{N}$.

Since $J_{0}^{w}$ relativises well, we can use $\mathcal{N}$ to find a $\mathcal{M}$ over $b$ satisfying subclaim 2.

This gives us the function $J_{1}$ as required by our claim. Notice also that the proof has shown that the $H\left(\omega_{1}\right)$ cones over $x$ of $V[g]$ and $V[G][g]$ are closed under $J_{1}$, and that

$$
J_{1}=J_{0}^{w}
$$

holds in $V[g]$ and $V[G][g]$ as well. This is what we need to obtain $J_{2}$ by the same method. We get $J_{1}^{w}$ on $H\left(\omega_{1}\right)^{V[G][g]}$ using $W_{\alpha+1}^{*}$ there. The $J_{1}$-closure of $V[h]$ and homogeneity of $\operatorname{Col}\left(\omega, \omega_{2}\right)$ give us $J_{1}^{w}$ on $H\left(\omega_{1}\right)^{V[g]}$ in $V[g]$. We get $I$ in $V$ such that $I(b)[g]=J_{1}^{w}(\langle b, g\rangle)$. $I$ extends to an $\left(\omega_{3}, \tau\right)$ operator in $V$, where $\tau_{g}=x$. Going back to $V[h]$, we can convert $I$ into the desired $J_{1}^{w}$-like function $J_{2}$.

We leave any further detail to the reader. This proves the claim.

We can now finish the proof of lemma 50. Let $J_{n}$ be the function in $V[h]$ we constructed. In $V[g]$, the $J_{n}(b)$ for $b$ in the $H\left(\omega_{1}\right)$ cone over $x$ collectively witness $W_{\alpha+1}^{*}$. This is because, as in the proof of 49 , we can reduce $\Sigma_{n}$ truth about $b$ in $J_{\alpha}(\mathbb{R})^{V[g]}$ to $\Sigma_{1}$ truth in $J_{n}(b)$. We get $I_{\alpha+1}$ because, as a byproduct of our construction, $J_{n} \cap H\left(\omega_{1}\right)^{V[g]}$ is contained in $\left(\left(J_{0}\right)^{w, \ldots, w}\right)^{V[G][g]}$. The former captures truth over $J_{\alpha}(\mathbb{R})^{V[g]}$, and the latter captures truth over $J_{\alpha}(\mathbb{R})^{V[G][g]}$. This implies that $\pi$ is fully elementary, as desired.

\section{The Admissible Cases}

Let us fix $g \subset \operatorname{Col}\left(\omega, \omega_{1}\right)$ which is $V$-generic, and a critical ordinal $\gamma$ in $V[g]$ of type (3). That is, letting $\alpha$ be the strict sup of the critical ordinals $<\gamma$, we have $\alpha<\gamma$. We assume that $W_{\alpha}^{*}$ holds in $V[g]$, and we wish to show that $W_{\gamma+1}^{*}$ holds in $V[g]$. The analysis of scales in $L(\mathbb{R})$ shows that $\alpha$ begins a $\Sigma_{1}$ gap $[\alpha, \beta]$, and $J_{\alpha}(\mathbb{R})$ is admissible. The possibilities are that $\alpha=\beta=\gamma-1$ (the admissible empty gap), that $\alpha<\beta=\gamma-1$ (the strong gap), or that $\alpha<\beta=\gamma$ (the weak gap). But for the most part, we do not need to distinguish these three cases here. We also assume $\operatorname{WRP}_{(2)}\left(\omega_{2}\right)$, and that $N S$ is saturated. Our overall plan is: 
Step 1. Working in $V[g]$, we construct a mouse $N$ and iteration strategy $\Sigma^{g}$ which code up truth at the end of the gap $[\alpha, \beta] . N$ will be a mouse over some real parameter $z$.

Step 2. Letting $\tau_{g}=z$, we show that $N$ and $\Sigma^{g}$ yield a mouse $N_{\tau}$ over $\tau$ and an $\omega_{2}$-iteration strategy $\Sigma$ for $N_{\tau}$, both in $V$, via the equations $N_{\tau}[g]=N$, and $\Sigma=\Sigma^{g} \uparrow V$.

Step 3. We show that $\Sigma$ extends to act on $H\left(\omega_{3}\right)$.

Step 4. We then further extend $\Sigma$ so that it acts on all trees in the $H\left(\omega_{1}\right)$ of $V[g][h]$, where $h \subset \operatorname{Col}\left(\omega, \omega_{2}^{V}\right)$ is $V[g]$-generic. At the same time we find versions $\left[\alpha^{H}, \beta^{H}\right]$ of our gap $[\alpha, \beta]$ in $V[g][H]$, whenever $H \in V[g][h]$, along with appropriately elementary embeddings from $J_{\beta}(\mathbb{R})^{V[g]}$ to $J_{\beta^{H}}(\mathbb{R})^{V[g][H]}$.

Step 5. We proceed as in the inadmissible case, but using $\Sigma$-mice with Woodin cardinals to witness $W_{\gamma+1}^{*}$ in $V[g]$. As before, the proof breaks into cases, according to whether $\alpha \in \operatorname{ran}\left(\pi_{\mathrm{NS}}\right.$ or not.

Steps 1 and 2 follow [22] closely. The only difference here is that we want $N$ to have $\omega$ Woodin cardinals, so that we can lift the gap $[\alpha, \beta]$ to $V[g][h]$, for $h$ generic over $\operatorname{Col}\left(\omega, \omega_{2}\right)$, using an $\mathbb{R}$-genericity iteration over $V[g][h]$. We now proceed to details.

Definition 51. Let $\Gamma$ be the pointclass $\Sigma_{1}^{J_{\alpha}(\mathbb{R})}$. What is called the envelope of $\Gamma$, or $\operatorname{ENV}(\Gamma)$, is the class of all $A \subseteq \mathbb{R}$ which are countably captured by $\Gamma$ in that there is a real $x$ such that for any countable $\sigma \subseteq \mathbb{R}, A \cap \sigma$ is $O D^{<\alpha}(\sigma, x)$. The analysis of scales from [23] shows that if $\alpha=\beta$ or $[\alpha, \beta]$ is strong, then

$$
\operatorname{ENV}(\Gamma)=J_{\beta+1}(\mathbb{R}) \cap P(\mathbb{R}),
$$

and if $[\alpha, \beta]$ is weak, then

$$
\operatorname{ENV}(\Gamma)=J_{\beta}(\mathbb{R}) \cap P(\mathbb{R}) .
$$

If $\alpha=\beta$ or $[\alpha, \beta]$ is strong, put

$$
e(\Gamma)=\left\{A \subseteq \mathbb{R} \mid A \text { is ordinal definable over } J_{\beta}(\mathbb{R})\right\} .
$$

If $[\alpha, \beta]$ is weak, put

$$
e(\Gamma)=\left\{A \subseteq \mathbb{R} \mid \exists \xi<\beta\left(\mathrm{A} \text { is ordinal definable over } J_{\xi}(\mathbb{R})\right\} .\right.
$$


So

$$
\operatorname{ENV}(\Gamma)=\bigcup_{z \in \mathbb{R}} e(\Gamma)(z)
$$

is the boldface pointclass associated to $e(\Gamma)$.

A 0 -suitable premouse is a minimal premouse $N$ with one $\Gamma$ Woodin, called $\delta^{N}$. Such an $N$ is $A$-iterable if it has a partial iteration strategy moving the $\operatorname{Col}\left(\omega, \delta^{N}\right)$ term relation for $A$ correctly. The reader should see [22] or [24] for full definitions. We have the following basic result of Woodin.

Theorem 52. (Woodin) For any countable transitive set $X$, and $A$ such that $A \in e(\Gamma)(z)$ for some $z \in X$, there is a 0 -suitable, A-iterable premouse over $X$.

No full proof of this key lemma has ever been written up. There is part of a proof in [16]. Thew paper [24] outlines a proof in the weak gap case.

If $[\alpha, \beta]$ is weak, we let $z_{0}$ be a real parameter such that for some finite set $F$ of ordinals, $\left\langle z_{0}, F\right\rangle$ satisfies a non-reflecting $\Sigma_{n}$ type, where $n$ is least such that $\rho_{n}\left(J_{\beta}(\mathbb{R})\right)=\mathbb{R}$. We let $F_{0}$ be the Brouwer-Kleene least such $F$, and let

$$
J_{\beta}(\mathbb{R})=\bigcup_{n} H_{n}
$$

be the decompostion given in [23]. Thus in particular each $H_{n}$ collapses to a member of $J_{\beta}(\mathbb{R})$. If $[\alpha, \beta]$ is not weak, let $z_{0}$ be a real such that for some $\Sigma_{1}$ formula $\varphi(v)$, we have

$$
J_{\beta+1}(\mathbb{R}) \models \varphi\left[z_{0}\right] \text { but } J_{\beta}(\mathbb{R}) \not \models \varphi\left[z_{0}\right] .
$$

Let $\rho$ be a standard $\operatorname{Col}\left(\omega, \omega_{1}^{V}\right)$ term for a real such that $\rho_{g}=z_{0}$, and let $p_{0}$ force all the properties of $\rho$ we have enumerated so far. For $p \in \operatorname{Col}\left(\omega, \omega_{1}^{V}\right)$ such that $p_{0} \subseteq p$, let $g_{p}(n)=p(n)$ if $n \in \operatorname{dom}(p)$, and $g_{p}(n)=g(n)$ otherwise. Let $\tau$ be a term for a real such that $\tau_{g}$ codes $\rho_{g}$ and $g$ in some natural way. It is easy to do this so that

(a) $z_{0} \leq_{T} \tau_{g}$, and

(b) for all $p, \tau_{g} \equiv_{T} \tau_{g_{p}}$.

Put $z=\tau_{g}$. For any $A \in e(\Gamma)(z)$, put

$B(A)=\{(y, t) \in \mathbb{R} \times \mathbb{R} \mid y$ codes a countable, transitive $X$ such that $z \in X$, and $t$ codes $\operatorname{Th}_{\omega}^{N}\left(X \cup\left\{X, \tau_{A}^{N}\right\}\right)$,

for some (all) 0-suitable, $A$-iterable $X$-premouse $N$ \}.

Here $\tau_{A}^{N}$ is the standard $\operatorname{Col}\left(\omega, \delta^{N}\right)$ - term capturing $A$. "Some" is equivalent to "all" in the definition above because $A$-iterability yields 
an approximation to the comparison process which suffices to determine the theory in question. Note that $B(A) \in e(\Gamma)(z)$, because $e(\Gamma)(z)$ is closed under real quantification. By the scale analysis of [23], we have a self-justifying system $\mathcal{A}=\left\{A_{i} \mid i<\omega\right\}$ such that

(1) each $A_{i}$ is in $e(\Gamma)(z)$,

(2) if $\alpha=\beta$ or $[\alpha, \beta]$ is strong, then for each $n<\omega, \operatorname{Th}_{n}^{J_{\beta}(\mathbb{R})}(\mathbb{R}) \in \mathcal{A}$

(3) if $[\alpha, \beta]$ is weak, then for all $n, \operatorname{Th}_{\omega}^{H_{n}}\left(\mathbb{R} \cup\left\{z, F_{0}\right\}\right) \in \mathcal{A}$, and

(4) for any $n, B\left(\left\langle A_{i} \mid i \leq n\right\rangle\right) \in \mathcal{A}$, where we regard $\left\langle A_{i} \mid i \leq n\right\rangle$ as a set of reals via some natural coding.

It is easy to also arrange that there is a fixed term $\dot{\mathcal{A}}$ such that

(5) for all $p \supseteq p_{0}, \dot{\mathcal{A}}^{g_{p}}=\dot{\mathcal{A}}^{g}$.

Let $X$ be countable transitive, with $z \in X$. Let $N^{n}$ be a 0 -suitable, $\left\langle A_{i} \mid i \leq n\right\rangle$-iterable mouse over $X$. As in [22] we can simultaneously compare all the $N^{n}$ to get a 0 -suitable $N$ over $X$ such that $N$ is $\left\langle A_{i}\right| i \leq$ $n\rangle$-iterable for all $n$. But then condensation for term relations implies that $N$ has a unique fullness preserving $\left(\omega_{1}, \omega_{1}\right)$ iteration strategy which moves all the term relations $\tau_{A_{i}}^{N}$ correctly. $^{8}$ Put

$$
Q(X)=\operatorname{Hull}^{N}\left(X \cup\{X\} \cup\left\{\tau_{A}^{N} \mid A \in \mathcal{A}\right\}\right) .
$$

Condensation for term relations of a self-justifying system implies that $Q(X)$ has all the properties of $N$, namely, it is 0 -suitable, and has a unique fullness preserving $\left(\omega_{1}, \omega_{1}\right)$ iteration strategy which moves all the term relations $\tau_{A_{i}}^{Q(X)}$ correctly. Moreover, $Q(X)$ is "sound", in that

$$
Q(X)=\operatorname{Hull}^{Q(X)}\left(X \cup\{X\} \cup\left\{\tau_{A}^{Q(X)} \mid A \in \mathcal{A}\right\}\right) .
$$

Let

$$
N_{0}=Q\left(V_{\omega} \cup\{z\}\right)
$$

and

$$
N_{k+1}=Q\left(N_{k}\right)
$$

and

$$
N=\bigcup_{k<\omega} N_{k}
$$

Put $\delta_{k}^{N}=\delta^{N_{k}}$. We regard $N$ as a premouse over $z$ in the natural way. Note that because $N_{k}$ is suitable, and hence $\Gamma$-full, no level of $N_{k+1}$ projects across $o\left(N_{k}\right)$, and thus the $\delta_{k}$ are all Woodin in $N$.

Lemma 53. There is a unique $\mathcal{A}$-guided strategy for $N$ in $V[g]$.

\footnotetext{
${ }^{8}$ See [22]. The strategy chooses the limit over $n$ of branches $b_{n}$ moving all $\tau_{A_{i}}^{N}$ for $i \leq n$ correctly.
} 
Proof. As in [22], there is a unique $\mathcal{A}$-guided iteration strategy $\Sigma_{0}$ for $N_{0}$. Let

$$
i: N_{0} \rightarrow S_{0}
$$

be an iteration map by $\Sigma_{0}$. We can let $i$ act on all of $N$, giving rise to

$$
i: N \rightarrow S \text {. }
$$

Put also $S_{m}=i\left(N_{m}\right)$, for all $m$. We do not yet know that $S$ is even wellfounded, but in fact

Claim 54. For all $m, S_{m+1}=Q\left(S_{m}\right)$.

Proof. We prove it for $m=0$. Let

$$
W_{k}=\operatorname{Th}_{\omega}^{N_{1}}\left(N \mid \delta \cup\left\{\tau_{A_{0}}^{N_{1}}, \ldots, \tau_{A_{k}}^{N_{1}}\right\}\right),
$$

where $\delta=\delta_{0}^{N}$. Note $W_{k} \in N_{0}$ because $N_{0}$ is $\Gamma$-full. Let

$$
B=B\left(\left\langle A_{0}, \ldots, A_{k}\right\rangle\right) \text {. }
$$

Now $N_{0}$ satisfies the sentence "it is forced in $\operatorname{Col}(\omega, \delta)$ that if $y$ codes $N \mid \delta$ and $t$ codes $W_{k}$, then $(y, t) \in \tau_{B}^{N_{0}}$." Thus the same sentence is true of $i(\delta), i\left(W_{k}\right)$, and $i\left(\tau_{B}^{N_{0}}\right)$ in $S_{0}$. But $i\left(\tau_{B}^{N_{0}}\right)=\tau_{B}^{S_{0}}$, and so

$$
\begin{aligned}
\operatorname{Th}_{\omega}^{S_{1}}\left(S \mid i(\delta) \cup\left\{\tau_{A_{0}}^{S_{1}}, \ldots, \tau_{A_{k}}^{S_{1}}\right\}\right) & =i\left(W_{k}\right) \\
& =\operatorname{Th}_{\omega}^{Q\left(S_{0}\right)}\left(S \mid i(\delta) \cup\left\{\tau_{A_{0}}^{Q\left(S_{0}\right)}, \ldots, \tau_{A_{k}}^{Q\left(S_{0}\right)}\right\}\right) .
\end{aligned}
$$

It follows that there is a natural isomorphism between

$$
\operatorname{Hull}_{\omega}^{S_{1}}\left(S \mid i(\delta) \cup\left\{\tau_{A_{0}}^{S_{1}}, \ldots, \tau_{A_{k}}^{S_{1}}\right\}\right)
$$

and

$$
\operatorname{Hull}_{\omega}^{Q\left(S_{0}\right)}\left(S \mid i(\delta) \cup\left\{\tau_{A_{0}}^{Q\left(S_{0}\right)}, \ldots, \tau_{A_{k}}^{Q\left(S_{0}\right)}\right\}\right) .
$$

Moreover, these isomorphisms commute with the inclusion maps on the hulls, because they are determined by the $i\left(W_{k}\right)$. Finally, $S_{1}$ and $Q\left(S_{0}\right)$ are the unions of the respective sequences of hulls, as $k$ varies. (In the case of $S_{1}$, this is because $N_{1}=Q\left(N_{0}\right)$, and $i$ came from an iteration based on $N_{0}$.) Thus $S_{1} \cong Q\left(S_{0}\right)$. The proof for $m>0$ is the same.

But now $S_{1}=Q\left(S_{0}\right)$ has a unique iteration strategy $\Sigma_{1}$ for trees above $S_{0}$. Letting $i: S \rightarrow T$ come from an iteration of $S_{1}$ by this strategy, and $T_{m}=i\left(S_{m}\right)$ we get $T_{m+1}=Q\left(T_{m}\right)$ for all $m \geq 1$ by repeating the proof of the claim above. We can then move on to iterating $T_{2}$ above $T_{1}$, etc. Clearly, this describes an iteration strategy for $N$ acting on normal trees. ${ }^{9}$

\footnotetext{
${ }^{9}$ In fact our strategy applies to trees of the form: a stack of normal trees below the first Woodin, then a stack of normal trees between the first and second Woodin, then a stack between the second and third, etc.
} 
$N$ is a mouse over $\tau_{g}$, but it can be re-arranged as a mouse over $\tau_{g_{p}}$ whenever $p \supseteq p_{0}$. The re-arranged mouse has the same universe and extender sequence; it just has a different (but Turing equivalent) real distinguished at the bottom. What is more, we have a fixed term $\dot{N}$ such that for all $p \supseteq p_{0}, \dot{N}_{g_{p}}$ is the re-arrangement of $N$ as a mouse over $\tau_{g_{p}}$. This is because of the symmetry in the construction of $N$, and in particular, because $\dot{\mathcal{A}}_{g}=\dot{\mathcal{A}}_{g_{p}}$ for all such $p$. This enables us to build in $V$ a premouse $N_{\tau}$ over $\tau$ such that $N_{\tau}[g]=N$. We construct $N_{\tau} \mid \alpha$ by induction on $\alpha$, maintaining that

$$
\left(N_{\tau} \mid \alpha\right)[g]=N \mid \alpha,
$$

along with the correspondence of projecta and parameters. $\alpha$ is active in $N_{\tau}$ iff it is active in $N$, and if so,

$$
E_{\alpha}^{N_{\tau}}=E_{\alpha}^{N} \cap N_{\tau} \mid \alpha .
$$

$N_{\tau} \mid(\alpha+1) \in V$ because by induction, $N_{\tau} \mid \alpha \in V$, and because $E_{\alpha}^{N}$ is independent of $g . E_{\alpha}^{N_{\tau}}$ is an extender over $N_{\tau}$ because $g$ was generic over $V$, and the forcing is small. The reader can find all the details of this construction in [24]. Let $\Sigma^{g}$ be the unique iteration strategy for $N$ given by the Lemma. Iterating $N_{\tau}$ is the same as iterating $N_{\tau}[g]=N$, because the forcing is small, and thus we can regard $\Sigma^{g}$ as a strategy for $N_{\tau}$. Moreover $\Sigma$, which denotes $\Sigma^{g} \uparrow V$, is in $V$ by the symmetry in our construction of $\Sigma^{g}$. Since $\Sigma^{g}$ condenses well, $\Sigma$ condenses well. We have finished steps 1 and 2 of the general plan.

We now execute step 3. Here we use $\operatorname{WRP}_{(2)}\left(\omega_{2}\right)$ in $V$ to extend our $\omega_{2}$-iteration strategy to an $\omega_{3}$-iteration strategy. In fact, simultaneous stationary reflection for pairs of subsets of $\omega_{2}$ is enough.

Lemma 55. Let $M$ be a premouse of cardinality $\leq \omega_{1}$, and let $\Sigma$ be any $\omega_{2}$ iteration strategy for $M$ which condenses well. Suppose that whenever $S, T \subseteq \omega_{2}$ are stationary and consist of ordinals of countable cofinality, there is a $\nu<\omega_{2}$ such that $S$ and $T$ are stationary in $\nu$. Then there is a unique $\omega_{3}$ iteration strategy $\Omega$ for $M$ such that

(1) $\Sigma \subseteq \Omega$, and

(2) $\Omega$ condenses well.

Proof. We omit the easy proof that there is at most one such $\Omega$. Fix $\eta$ large. Let $\mathcal{T}$ be an iteration tree on $M$ with $\operatorname{lh}(\mathcal{T})<\omega_{3}$. We say $\left\langle X_{\alpha} \mid \alpha<\omega_{2}\right\rangle$ is a $\mathcal{T}$-chain iff

(a) $X_{\alpha} \prec V_{\eta}$, for all $\alpha<\omega_{2}$,

(b) $\alpha<\beta \Rightarrow X_{\alpha} \subsetneq X_{\beta}$, and $X_{\lambda}=\bigcup_{\alpha<\lambda} X_{\alpha}$ for limit $\lambda$,

(c) $M, \mathcal{T} \in X_{0}$, and

(d) $\left|X_{\alpha}\right|=\omega_{1}$, and $X_{\alpha} \cap \omega_{2} \in \omega_{2}$, for all $\alpha<\omega_{2}$. 
Given a $\mathcal{T}$-chain $\vec{X}$, we let $\pi_{\alpha}: H_{\alpha} \cong X_{\alpha}$ with $H_{\alpha}$ transitive, let $\pi_{\alpha, \beta}=$ $\pi_{\beta}^{-1} \circ \pi_{\alpha}$, and let $\mathcal{T}_{\alpha}=\pi_{\alpha}^{-1}(\mathcal{T})$. We say that $\vec{X}$ is $\Sigma$-good iff each $\mathcal{T}_{\alpha}$ is by $\Sigma$, and in that case, we set

$$
b_{\alpha}=\Sigma\left(\mathcal{T}_{\alpha}\right)
$$

for all $\alpha<\omega_{2}$. There is of course no reason that we should have $b_{\alpha} \in H_{\alpha}$.

Claim 56. Let $\vec{X}$ be a $\Sigma$-good $\mathcal{T}$-chain, and let $\gamma<\omega_{2}$ with $\operatorname{cof}(\gamma)=$ $\omega_{1}$; then for club many $\alpha<\gamma, \pi_{\alpha, \gamma} " b_{\alpha} \subseteq b_{\gamma}$.

Proof. We take cases on the cofinality of the length of $\mathcal{T}$. Suppose first $\operatorname{cof}(\operatorname{lh}(\mathcal{T}))=\omega$. Then for all sufficiently large $\alpha<\gamma, \operatorname{ran}\left(\pi_{\alpha, \gamma}\right)$ is cofinal in $b_{\gamma}$, and thus applying condensation to the support-closed subtree of $\mathcal{T}_{\gamma} \frown b_{\gamma}$ determined by $\operatorname{ran}\left(\pi_{\alpha, \gamma}\right)$, we get that $\pi_{\alpha, \gamma}^{-1}$ " $b_{\gamma}=\Sigma\left(\mathcal{T}_{\alpha}\right)=b_{\alpha}$. So the desired club is just a tail below $\gamma$. Suppose $\operatorname{cof}(\operatorname{lh}(\mathcal{T}))=\omega_{1}$. Then $\operatorname{cof}\left(\operatorname{lh}\left(\mathcal{T}_{\xi}\right)\right)=\omega_{1}$, for all $\xi$. Also, for all $\alpha<\gamma, \pi_{\alpha, \gamma}$ " $b_{\alpha}$ is cofinal in $l h\left(\mathcal{T}_{\gamma}\right)$. Since $\mathcal{T}_{\gamma}$ has at most one cofinal branch, we get $\pi_{\alpha, \gamma}{ }^{\prime \prime} b_{\alpha} \subseteq b_{\gamma}$. Finally, suppose $\operatorname{cof}(\operatorname{lh}(\mathcal{T}))=\omega_{2}$. As in case two, $\operatorname{cof}\left(\operatorname{lh}\left(\mathcal{T}_{\xi}\right)\right)=\operatorname{cof}\left(X_{\xi} \cap \omega_{2}\right)$, , for all $\xi$, but now, $\alpha<\gamma \Rightarrow \pi_{\alpha, \gamma}$ is discontinuous at $l h\left(\mathcal{T}_{\alpha}\right)$. Fixing $\gamma$ with $\operatorname{cof}(\gamma)=\omega_{1}$, we can find club many $\alpha<\gamma$ such that $\operatorname{ran}\left(\pi_{\alpha, \gamma}\right) \cap b_{\gamma}$ is cofinal in $\sup \pi_{\alpha, \gamma} " l h\left(\mathcal{T}_{\alpha}\right)$. For any such $\alpha$, condensation for the support-closed subtree of $\mathcal{T}_{\gamma} b_{\gamma}$ determined by $\operatorname{ran}\left(\pi_{\alpha, \gamma}\right)$, implies that $\pi_{\alpha, \gamma}^{-1}$ " $b_{\gamma}=\Sigma\left(\mathcal{T}_{\alpha}\right)=b_{\alpha}$.

Let $\vec{X}$ be a $\Sigma$-good $\mathcal{T}$-chain. We say $\vec{X}$ is coherent if and only if whenever $\alpha<\gamma<\omega_{2}$, then $\pi_{\alpha, \gamma}$ " $b_{\alpha} \subseteq b_{\gamma}$. In this case, we say $\vec{X}$ justifies $b$, where

$$
b=\bigcup_{\alpha<\omega_{2}} b_{\alpha} .
$$

It is easy to see

Claim 57. $\mathcal{T}$ has at most one branch $b$ which is justified by some coherent $\mathcal{T}$-chain.

Proof. If $\vec{X}$ and $\vec{Y}$ are $\Sigma$-good $\mathcal{T}$-chains, then for club many $\alpha<\omega_{2}$, $X_{\alpha} \cap \omega_{2}=Y_{\alpha} \cap \omega_{2}$. Thus for club many $\alpha<\omega_{2}, \mathcal{T}_{\alpha}^{\vec{X}}=\mathcal{T}_{\alpha}^{\vec{Y}}$ and $b_{\alpha}^{\vec{X}}=b_{\alpha}^{\vec{Y}}$.

So we define

$$
\Omega(\mathcal{T})=b \Leftrightarrow \mathrm{b} \text { is justified by some coherent } \mathcal{T} \text {-chain. }
$$

Claim 58. If $\mathcal{T}$ is by $\Omega$, then every $\mathcal{T}$-chain is $\Sigma$-good. 
Proof. Let $\mathcal{T}$ be of minimal length such that the claim is false. Suppose first that $l h(\mathcal{T})$ is a limit ordinal. Let $\vec{X}$ be a $\mathcal{T}$-chain. If $\alpha<\gamma<\omega_{2}$, then $\mathcal{T}_{\alpha}$ is the collapse of a support-closed subtree of $\mathcal{T}_{\gamma}$, so since $\Sigma$ condenses well, we have that $\mathcal{T}_{\gamma}$ is not by $\Sigma$ for all sufficiently large $\gamma<\omega_{2}$. Using a surjective map $f: \omega_{2} \rightarrow \operatorname{lh}(\mathcal{T})$ with $f \in X_{0}$, and a Fodor argument, we can fix $\xi<l h(\mathcal{T})$ such that for stationary many $\alpha<\omega_{2}, \pi_{\alpha}^{-1}(\mathcal{T} \uparrow \xi)$ is not by $\Sigma$. But $\vec{X}$ is a $\mathcal{T} \uparrow \xi$-chain, contrary to the minimality of $\operatorname{lh}(\mathcal{T})$. Thus $\ln (\mathcal{T})=\lambda+1$ for some $\lambda$. It is clear that $\lambda$ must be a limit ordinal. Let $b=\Omega(\mathcal{T} \mid \lambda)$, and let $\vec{X}$ be a $\mathcal{T}$-chain. Let $\vec{Y}$ be a $\mathcal{T} \uparrow \lambda$-chain which justifies $b$. There are club many $\alpha<\omega_{2}$ such that $X_{\alpha} \cap \operatorname{lh}(\mathcal{T})=Y_{\alpha} \cap \operatorname{lh}(\mathcal{T})$, and for such $\alpha,\left(\pi_{\alpha}^{\vec{X}}\right)^{-1}(b)=\left(\pi_{\alpha}^{\vec{Y}}\right)^{-1} " b=b_{\alpha}^{\vec{Y}}$. Thus for club many $\alpha, \mathcal{T}_{\alpha}^{\vec{X}}$ is by $\Sigma$. Condensation implies this is true for all $\alpha$. This contradiction completes the proof.

Claim 59. $\Sigma \subseteq \Omega$, and $\Omega$ condenses well.

Proof. If $\mathcal{T}$ is by $\Sigma$, then in any $\mathcal{T}$-chain, we have $\mathcal{T}_{\alpha}=\mathcal{T}$ for all $\alpha<\omega_{2}$, so every $\mathcal{T}$-chain justifies $\Sigma(\mathcal{T})$. For condensation, suppose $\Omega(\mathcal{T})=b$, and $\mathcal{U} \frown c$ is the collapse of a support-closed subtree of $\mathcal{T}^{\frown} b$, and $\Omega(\mathcal{U})=d$ where $d \neq c$. It is easy to see that there is a single $\vec{X}$, with $\mathcal{T}, \mathcal{U}, b, c, d \in X_{0}$, which justifies both $b$ and $d$. But this gives a failure of condensation for $\Sigma$.

Claim 60. Suppose $\mathcal{T}$ is by $\Omega$, and $l h(\mathcal{T})<\omega_{3}$; then there is a $b$ such that $\Omega(\mathcal{T})=b$.

Proof. Fix any $\xi<l h(\mathcal{T})$, and any $\mathcal{T}$-chain $\vec{X}$. Since $\vec{X}$ is $\Sigma$-good, we have $b_{\alpha}=\Sigma\left(\mathcal{T}_{\alpha}\right)$ for $\alpha<\omega_{2}$. We claim that exactly one of the following holds:

(1) for $\omega$-club many $\alpha<\omega_{2}, \pi_{\alpha}^{-1}(\xi) \in b_{\alpha}$,

(2) for $\omega$-club many $\alpha<\omega_{2}, \pi_{\alpha}^{-1}(\xi) \notin b_{\alpha}$.

It is clear that not both can hold. Suppose both fail. Let $S$ be the stationary set of $\alpha$ of cofinality $\omega$ where $\xi \in \operatorname{ran}\left(\pi_{\alpha}\right)$ and (1) fails, and $T$ the stationary set of $\alpha$ of cofinality $\omega$ where (2) fails. By our stationary reflection hypothesis, we can fix $\gamma$ of cofinality $\omega_{1}$ such that both $S$ and $T$ are stationary in $\gamma$. Note $\xi \in \operatorname{ran}\left(\pi_{\gamma}\right)$. If $\pi_{\gamma}^{-1}(\xi) \in b_{\gamma}$, then by the first claim, $\pi_{\alpha}^{-1}(\xi) \in b_{\alpha}$ for club-in- $\gamma$ many $\alpha$, so $T$ was not stationary in $\alpha$, contradiction. Similarly, if $\pi_{\gamma}^{-1}(\xi) \notin b_{\gamma}$, then The first claim implies $S$ is not stationary in $\gamma$, a contradiction. So at least one of (1) and (2) holds. It also implies that the $\omega$-clubs of (1) and (2) can be taken to be fully club in $\omega_{2}$. Define $b$ by:

$$
\xi \in b \Leftrightarrow \text { for club many } \alpha<\omega_{2}, \pi_{\alpha}^{-1}(\xi) \in b_{\alpha} .
$$


Taking a diagonal intersection, we can find a club $C \subseteq \omega_{2}$ such that for $\alpha \in C, \pi_{\alpha} " b_{\alpha} \subseteq b$. But then $\left\langle X_{\alpha} \mid \alpha \in C\right\rangle$ is a coherent $\mathcal{T}$-chain which justifies $b$.

This completes the proof of Lemma 55.

Applying Lemma 55, let us use $\Sigma$ to denote the unique $\omega_{3}$ iteration strategy for $N_{\tau}$ which condenses well, and extends $\Sigma^{g} \uparrow V$. Proceeding to step 4 , we need to further extend $\Sigma$ so that it acts on all trees in $H\left(\omega_{1}\right)^{V[g][h]}$, whenever $h$ is $V[g]$-generic over some poset in $H\left(\omega_{2}\right)^{V[g]}$. These extensions of $\Sigma$ will be mutually consistent. At the same time, we will be showing that the gap $[\alpha, \beta]$ of $V[g]$ has counterparts in every $V[g][h]$. The following little lemma will be useful.

Lemma 61. Let $\Gamma$ be an iteration strategy for $S$ which condenses well. Let $\pi: R \rightarrow S$ be sufficiently elementary that the pullback strategy $\Gamma^{\pi}$ for $R$ exists; then $\Gamma^{\pi}$ also condenses well.

Proof. Let $\mathcal{T}$ be a tree according to $\Gamma^{\pi}$, and $\mathcal{U}$ a support-closed subtree of $\mathcal{T}$ corresponding to those $\mathcal{M}_{\alpha}^{\mathcal{T}}$ such that $\alpha \in X$, and let $\overline{\mathcal{U}}$ be the collapse of $\mathcal{U}$. It is easy to see the lifted tree $\pi \overline{\mathcal{U}}$ is the collapse of the support-closed subtree of $\pi \mathcal{T}$ corresponding to those $\mathcal{M}_{\alpha}^{\pi \mathcal{T}}$ such that $\alpha \in X$. Since $\Gamma$ condenses well, $\pi \overline{\mathcal{U}}$ is according to $\Gamma$, and hence $\overline{\mathcal{U}}$ is according to $\Gamma^{\pi}$.

We need to use hybrid strategy mice. Suppose $\Omega$ is an iteration strategy for some structure $M$, and $\Omega$ condenses well. Let $A$ be transitive, with $M \in A$. We obtain a hybrid $\Omega$-premouse by adding extenders with critical points above $A$ to a coherent sequence we are building, and at the same time closing the model we are building under $\Omega$, and giving it a predicate for $\Omega$. The construction can only go on as long as all (non-dropping) iteration trees according to $\Omega$ we construct are in the domain of $\Omega$. ( $M$ may or may not be a fine-structural premouse, but in any case, it is convenient to only close under $\Omega$ on non-dropping trees.) We refer the reader to [22] for a brief discussion of such $\Omega$-hybrids, and to $[16]$ for a more thorough one.

Definition 62. Let $\Omega$ be an $|A|^{+}$-iteration strategy for $M$ which condenses well, where $A$ is transitive and $M \in A$; then $P_{n}^{\Omega}(A)^{\sharp}$ is the minimal $|A|^{+}$-iterable hybrid $\Omega$ - mouse over $A$ which is active, and satisfies "there are $n$ Woodin cardinals".

We note that the iterations referred to here all leave $A$, and hence $M$, fixed. It is part of iterability that they must move $\Omega$ to itself. One can hope to construct such iterable hybrid mice in a $K^{c}$ construction, 
because $\Omega$ condenses well, and hence $\Omega$ will condense to itself under realizing maps. The iterability demand we have made for $P_{n}^{\Omega}(A)^{\sharp}$ in the definition above is the minimal one which guarantees uniqueness, granted that $H\left(|A|^{+}\right)$is closed under $P_{n-1}^{\Omega}$-sharps. We shall never consider a putative $P_{n}^{\Omega}(A)$-sharp unless we already know $H\left(|A|^{+}\right)$is closed under $P_{n-1}^{\Omega}$-sharps. In practice, we often have more iterability than the minimal demand. Our core-model-induction proof that $H\left(\omega_{3}\right)$ of $V$ is closed under the $M_{n}^{\sharp}$ operators generalizes routinely to hybrid mouse operators, and gives:

Lemma 63. Assume $N S$ is saturated, and $\mathrm{WRP}_{(2)}\left(\omega_{2}\right)$ holds. Let $S \in$ $H\left(\omega_{1}\right)$, and let $\Omega$ be an $\omega_{3}$-iteration strategy for $S$ which condenses well. Then for all transitive $A \in H\left(\omega_{3}\right)$ such that $S \in A$, and all $n<\omega$, $P_{n}^{\Omega}(A)^{\sharp}$ exists and is $\omega_{3}$-iterable.

This is proved exactly as was theoremjtojw, so we omit the details.

Lemma 63 is the place core model theory gives us new mice. We shall eventually apply it in the case that $\alpha \in \operatorname{ran}\left(\pi_{G}\right)$ for some NS-generic $G$. In that case, we can take our real $z_{0}$ to be in $V$, and $N_{\tau}$ to be in $V$, and countable there. (We don't actually need $\tau$ at all, $N$ could be a $z_{0}$-premouse.) We shall then apply lemma 63 with $S=N$ and $\Omega=\Sigma$.

Before we take cases on whether $\alpha \in \operatorname{ran}\left(\pi_{G}\right)$, however, we do some further preliminary work related to $I_{\gamma}$.

Lemma 64. For all $A \in H\left(\omega_{3}\right), P_{0}^{\Sigma}(A)^{\sharp}$ exists and is $\omega_{3}$-iterable.

Proof. We show first that $P_{0}^{\Sigma}(A)^{\sharp}$ exists for all such $A \in H\left(\omega_{2}\right)$, then extend this to $A \in H\left(\omega_{3}\right)$ using simultaneous reflection. Let $G \subset$ $P\left(\omega_{1}\right) / N S$ be $V$-generic and let

$$
i: V \rightarrow M \subseteq V[G]
$$

be the generic embedding. Since $N_{\tau} \in H\left(\omega_{2}\right)^{V}$, we have $N_{\tau} \in M$, and $i \uparrow N_{\tau} \in M$. So inside $M$, we can form the $\left(i \uparrow N_{\tau}\right)$-pullback of $i(\Sigma)$, which we denote by $i(\Sigma)^{i}$. From the point of view of $M, i(\Sigma)^{i}$ is an $\omega_{3}$ iteration strategy for $N_{\tau}$, and by Lemma 61, it condenses well in $M$.

Claim 65. $i(\Sigma)^{i}$ agrees with $\Sigma$ on all trees in the intersection of the two domains.

Proof. We first consider trees in $H\left(\omega_{2}\right)^{V}$, all of which are in both domains. Let $\mathcal{T} \in H\left(\omega_{2}\right)^{V}$ be a tree according to $\Sigma$. Note that $i \uparrow N_{\tau} \in M$. In $M$ the copied tree $i \mathcal{T}$ on $i\left(N_{\tau}\right)$ is satisfied to be the collapse of a support closed subtree $i(\mathcal{T})$. Since $i(\Sigma)$ condenses well in $M, i \mathcal{T}$ is according to $i(\Sigma)$. Hence $\mathcal{T}$ is according to the pullback $i(\Sigma)^{i}$. Now let $\mathcal{U}$ be a tree in $\mathrm{V}$ of size $\omega_{2}^{V}$ in $\mathrm{V}$ which is according 
to both $\Sigma$ and $i(\Sigma)^{i}$, and is of limit length. Let

$$
b=i(\Sigma)^{i}(\mathcal{U})
$$

and

$$
c=\Sigma(U)
$$

and

$$
b \neq c .
$$

Note that $c f(\operatorname{lh}(\mathcal{U}))$ must be countable in $V[G]$. In $\mathrm{V}$, we can write $\mathcal{U}=\bigcup_{\alpha<\omega_{2}} \mathcal{U}_{\alpha}$, where this is an increasing continuous chain of supportclosed subtrees, each of size $\omega_{1}$. Going to $\mathrm{V}[\mathrm{G}]$, where $c f(\operatorname{lh}(\mathcal{U}))$ is countable, we see that

$$
b \cap \mathcal{U}_{\alpha} \text { is cofinal in } \mathcal{U}_{\alpha}
$$

and

$$
c \cap \mathcal{U}_{\alpha} \text { is cofinal in } \mathcal{U}_{\alpha},
$$

for all sufficiently large $\alpha$, so we may assume all $\alpha$. Let $\overline{\mathcal{U}}_{\alpha}$ denote the collapse of $\mathcal{U}_{\alpha}, \bar{b}_{\alpha}$ the collapse of $b \cap \mathcal{U}_{\alpha}, \bar{c}_{\alpha}$ the collapse of $c \cap \mathcal{U}_{\alpha}$. Fix $\alpha$ such that $\bar{b}_{\alpha} \neq \bar{c}_{\alpha}$. Now

$$
\bar{c}_{\alpha}=\Sigma\left(\overline{\mathcal{U}}_{\alpha}\right)
$$

because $\Sigma$ condenses well in V. On the other hand,

$$
\bar{b}_{\alpha}=i(\Sigma)^{i}\left(\overline{\mathcal{U}}_{\alpha}\right) \text {, }
$$

because $i(\Sigma)^{i}$ condenses well in $M$. Since $\Sigma$ and $i(\Sigma)^{i}$ must agree at $\bar{U}_{\alpha}$ by the first part, we are done.

Now fix a transitive $A \in H\left(\omega_{2}\right)$ such that $N_{\tau} \in A$. Let $L^{\Sigma}[A]$ be the minimal model of height $\omega_{3}$ which has $A$ as a member and is closed under $\Sigma$, and is expanded by a predicate for $\Sigma$. In $M$, we can form $L^{i(\Sigma)^{i}}[A]$ in parallel fashion. By claim 65 , these two models are the same. So $L^{\Sigma}[A] \in M$. But $i(N S)$ is saturated in $M$, so by the same argument that shows that the existence of a saturated ideal implies $0^{\sharp}$ exists, we get some $P \in M$ such that

$$
M \models P=P_{0}^{\Sigma}(A)^{\sharp} .
$$

Being $P_{0}^{\Sigma}(A)^{\sharp}$ is a first order property, combined with linear iterability by the last (and only) extender in a way that moves $\Sigma$ to itself, for iterations of length $<\omega_{3}^{M}=\omega_{3}^{V}$. But now let $h$ be $V$-generic for $\operatorname{Col}\left(\omega, \omega_{2}^{V}\right)$, and such that $G \in V[h]$. The required iterability of $P$ is upward absolute, that is

$$
V[h] \models P \text { is } \omega_{3}^{V} \text { iterable, }
$$


so since $\omega_{3}^{V}$ is still uncountable in $V[h]$,

$$
V[h]=P=P_{0}^{\Sigma}(A)^{\sharp} .
$$

By the homogeneity of $\operatorname{Col}\left(\omega, \omega_{2}\right), P \in V$, and

$$
V \models P=P_{0}^{\Sigma}(A)^{\sharp},
$$

and we are done in the case $A \in H\left(\omega_{2}\right)$. We now use $\operatorname{WRP}_{(2)}\left(\omega_{2}\right)$, via hybrid mouse reflection at $\omega_{2}$, to show that $P_{0}^{\Sigma}(A)^{\sharp}$ exists, for all transitive $A \in H\left(\omega_{3}\right)$ such that $N_{\tau} \in A$. Without loss of generality, let us assume $A \subseteq \omega_{2}$. Let $\phi$ be a formula in the language of set theory together with a predicate symbol $\dot{\Sigma}$ and constant symbol $\dot{A}$, and let $\vec{\alpha} \in \omega_{2}^{<\omega}$. For $\sigma \prec H\left(\omega_{2}\right)$ countable, let

$$
\pi_{\sigma}: M_{\sigma} \rightarrow H_{\omega_{2}}
$$

be the transitive collapse, and $A_{\sigma}=\pi_{\sigma}^{-1}\left(A_{\sigma}\right), N_{\sigma}=\pi_{\sigma}^{-1}\left(N_{\tau}\right)$, and $\vec{\alpha}_{\sigma}=\pi_{\sigma}^{-1}(\vec{\alpha})$. Note that for such $\sigma$, the pullback strategy $\Sigma^{\pi_{\sigma}}$ is a full $\omega_{3}$ iteration strategy for $N_{\sigma}$, and it condenses well. Using our saturated ideal, we then have that

$$
P_{0}^{\Sigma^{\pi_{\sigma}}}\left(A_{\sigma}\right)^{\sharp} \text { exists, }
$$

and is $\omega_{3}$ iterable. We put

$$
\begin{aligned}
(\phi, \vec{\alpha}) \in P_{0}^{\Sigma}(A)^{\sharp} \Leftrightarrow \quad & \text { for club many } \sigma \in P_{\omega_{1}}\left(H_{\omega_{2}}\right) \\
& \left(\phi, \vec{\alpha}_{\sigma}\right) \in P_{0}^{\Sigma^{\pi_{\sigma}}}\left(A_{\sigma}\right)^{\sharp} .
\end{aligned}
$$

(Here we identify the structure $P_{0}^{\Sigma}(A)^{\sharp}$ with its theory with parameters from $\omega_{2}$.) In order to see that this definition works, we must show that every $(\phi, \vec{\alpha})$ is decided on a club. So suppose neither $(\phi, \vec{\alpha})$ nor $(\neg \phi, \vec{\alpha})$ is in $P_{0}^{\Sigma}(A)^{\sharp}$ according to the definition above. As usual, we find a transitive $X \prec H_{\omega_{2}}$ with $|X|=\omega_{1}$ such that both sets are stationary in $P_{\omega_{1}}(X)$. Without loss of generality, assume $\vec{\alpha}, N_{\tau} \in X$, and

$$
(\phi, \vec{\alpha}) \in P_{0}^{\Sigma}(A \cap X)^{\sharp} .
$$

It is then easy to see that for club many $\sigma \in P_{\omega_{1}}(X),\left(\phi, \vec{\alpha}_{\sigma}\right) \in$ $P_{0}^{\Sigma^{\pi_{\sigma}}}\left(A_{\sigma}\right)^{\sharp}$. That is because for club many $\sigma \prec X, \sigma=Z \cap X$ for some $Z \prec V_{\eta}$ with $P_{0}^{\Sigma}(A \cap X)^{\sharp} \in Z$. Letting $\pi \supseteq \pi_{\sigma}$ be the collapse of $Z$, we get that

$$
\pi^{-1}\left(P_{0}^{\Sigma}(A \cap X)^{\sharp}\right)=P_{0}^{\Sigma^{\pi_{\sigma}}}\left(A_{\sigma}\right)^{\sharp} .
$$

To see this, note $\pi^{-1}(\Sigma) \subseteq \Sigma^{\pi_{\sigma}}$ by our argument in the first part of the proof of 64 . So the strategy predicate in $\pi^{-1}\left(P_{0}^{\Sigma}(A \cap X)^{\sharp}\right)$ denotes $\Sigma^{\pi_{\sigma}}$. Moreover, iterates $S$ of $\pi^{-1}\left(P_{0}^{\Sigma}(A \cap X)^{\sharp}\right)$ embed into iterates $S^{*}$ of $P_{0}^{\Sigma}(A \cap X)^{\sharp}$, and the strategy predicate of $S^{*}$ denotes a fragment of $\Sigma$, so the strategy predicate of $S$ denotes a fragment of $\Sigma^{\pi_{\sigma}}$. So we 
have shown $(\phi, \vec{\alpha}) \in P_{0}^{\Sigma}(A)^{\sharp}$, or $(\neg \phi, \vec{\alpha}) \in P_{0}^{\Sigma}(A)^{\sharp}$. This easily gives that our $P_{0}^{\Sigma}(A)^{\sharp}$ has the first order properties required of $P_{0}^{\Sigma}(A)^{\sharp}$.

We must see that its strategy predicate denotes $\Sigma$, and that linear iterates of it move $\Sigma$ correctly. Let $I$ be a linear iteration of length $<\omega_{3}$ of $P$, with last model $Q$ such that $\dot{\Sigma}^{Q} \nsubseteq \Sigma$. We can find

$$
\pi: H \rightarrow V_{\eta}
$$

such that $M$ is countable transitive, and everything relevant is in $\operatorname{ran}(\pi)$. Because $\operatorname{ran}(\pi) \cap \omega_{2}$ meets the clubs definable over $V_{\eta}$ from elements of $\operatorname{ran}(\pi)$, we get

$$
\pi^{-1}(P)=P_{0}^{\Sigma^{\pi}}\left(\pi^{-1}(A)\right)^{\sharp} .
$$

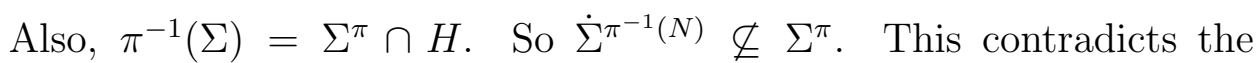
fact that linear iterations of $P_{0}^{\Sigma^{\pi}}\left(\pi^{-1}(A)\right)^{\sharp} d o$ move $\Sigma^{\pi}$ to itself, by definition.

We shall now use genericity iterations of $N_{\tau}$ to lift $J_{\beta}(\mathbb{R})^{V[g]}$ and $\Sigma^{g}$ to $V[g][h]$, for any $h$ generic over $V[g]$ for a poset in $H\left(\omega_{2}\right)^{V[g]}$. To this end, recall our self-justifying system $\mathcal{A}=\left\{A_{i} \mid i<\omega\right\}$ in $V[g]$. For $A \in \mathcal{A}$ and $\delta$ a Woodin cardinal of $N$, we have the $\operatorname{Col}(\omega, \delta)$-term $\tau_{A, \delta}^{N}$, whose images in $\Sigma^{g}$-iterations always capture $A$. Since $N=N_{\tau}[g]$, we have $\tau_{A, \delta}^{N}=\rho_{g}$ for some $\operatorname{Col}\left(\omega, \omega_{1}^{V}\right)$ - term $\rho$. Let $\sigma_{A, \delta}$ be the canonical $\operatorname{Col}\left(\omega, \omega_{1}^{V}\right) \times \operatorname{Col}(\omega, \delta)$ term such that for all generics $k \times l$,

$$
\left(\sigma_{A, \delta}\right)_{k \times l}=\left(\rho_{k}\right)_{l} .
$$

Thus

$$
\left(\sigma_{A, \delta}\right)_{g \times l}=\left(\tau_{A, \delta}^{N}\right)_{l}
$$

for $l$ being $\operatorname{Col}(\omega, \delta)$ generic over $N_{\tau}[g]$.

Lemma 66. Let $h \subset \mathbb{P}$ be $V[g]$-generic where $\mathbb{P} \in\left(H\left(\omega_{3}^{V}\right)\right)^{V[g]}$. Then in $V[g][h]$ there are

(1) sets $A_{i}^{*} \subseteq \mathbb{R}$ such that

$$
\left(H C^{V[g]}, \in, A_{i}\right)_{i<\omega} \prec\left(H C^{V[g][h]}, \in, A_{i}^{*}\right)_{i<\omega},
$$

(2) an ordinal $\beta^{h}$ and embedding

$$
\pi: J_{\beta}(\mathbb{R})^{V[g]} \rightarrow J_{\beta^{h}}(\mathbb{R})^{V[g][h]}
$$

such that $\pi$ is fully elementary if $\alpha=\beta$ or $[\alpha, \beta]$ is strong, and $\pi$ is $\Sigma_{n}$-elementary for $n$ least such that $\rho_{n}\left(J_{\beta}(\mathbb{R})^{V[g]}\right)=\mathbb{R}$ otherwise, and

(3) a unique $\omega_{3}^{V}$-iteration strategy $\Sigma^{h} \in V[g][h]$ for $N$ which extends $\Sigma^{g} \cup \Sigma$ and condenses well. 
Proof. We begin with (1). $A_{i}^{*}$ comes from interpreting the images of $\tau_{A_{i}}^{N_{\tau}}$ under genericity iterations. Note first

Claim 67. In $V$, let $M \in H\left(\omega_{3}\right)$ be any non-dropping $\Sigma$-iterate of $N_{\tau}$, and let $k<\omega$. Let $x \in \mathbb{R} \cap V[g][h]$; then there is (in $V$ ) a $\Sigma$-iteration map $i: M \rightarrow P$ with $\operatorname{crit}(i)>\delta_{k}^{M}$ such that for any $\operatorname{Col}\left(\omega, \delta_{k}^{M}\right)$-generic $l$ over $P$ such that $l \in V[g][h]$ and $g, h \in P[l]$, we have that $x \in P[l][f]$, for some $f \in V[g][h]$ such that $f$ is $\operatorname{Col}\left(\omega, \delta_{k+1}^{P}\right)$-generic over $P$.

Proof. Let $x=\sigma_{g \star h}$. Working in $V$, we use the standard genericity iteration for the $\omega_{2}^{V}$-generator version of the extended algebra of $M$ at $\delta_{k+1}^{M}$ to make $\sigma$ generic. We get in $V$ an $i: M \rightarrow P$ with $\operatorname{crit}(i)>\delta_{k}^{M}$ such that for any $\operatorname{Col}\left(\omega, \delta_{k}^{M}\right)$-generic $l$ over $P$, there is $f$ as in our claim with $\sigma \in P[l][f]$. So if $g, h \in P[l]$, then $x \in P[l][f]$. The important thing to note is that the genericity iteration yielding $i$ terminates. This follows from the fact that $P^{\Sigma}(C)^{\sharp}$ exists, where $C \in H\left(\omega_{3}\right)$ codes up $\sigma$ and the iteration from $N_{\tau}$ to $M$.

Claim 68. Let $i: N_{\tau} \rightarrow P$ and $j: N_{\tau} \rightarrow Q$ be non-dropping $\Sigma$-iterations of $N_{\tau}$ (in $V$ ), and let $\delta$ and $\mu$ be Woodin cardinals of $N_{\tau}$. Let $A \in \mathcal{A}$ be in our self-justifying system from $V[g]$. Let $x \in \mathbb{R}^{V[g][h]}$ be such that

$$
x \in P[g]\left[l_{0}\right] \cap Q[g]\left[l_{1}\right],
$$

where $l_{0}, l_{1}$ are generic over $P[g], Q[g]$ for the collapses of $i(\delta)$ and $j(\mu)$, respectively. Then

$$
x \in i\left(\sigma_{A, \delta}\right)_{g \times l_{0}} \Leftrightarrow x \in j\left(\sigma_{A, \mu}\right)_{g \times l_{1}} .
$$

Proof. If not, we have $(p, q) \in g \star h$ such that

$$
(p, q) \Vdash \phi\left(\check{N}_{\tau}, \check{\Sigma}\right)
$$

where $\phi$ in the language for forcing over $V$ expresses the failure of out claim in $V[g][h]$. Here $\phi$ also involves check-names for $\sigma_{A, \delta}$ and $\sigma_{A, \mu}$, which we have suppressed. In $V$, let

$$
\pi: H \rightarrow V_{\eta},
$$

where $H$ is transitive and of size $\omega_{1}, \omega_{1} \in H$, and everything relevant is in $\operatorname{ran}(\pi)$. We can find

$$
\bar{h} \in V[g]
$$

so that

$$
g \star \bar{h} \text { is } \operatorname{Col}\left(\omega, \omega_{1}\right) \star \pi^{-1}(\dot{\mathbb{P}}) \text {-generic over } H,
$$

and $\pi^{-1}(q) \in \bar{h}$. Note that by condensation for $\Sigma$,

$$
\pi^{-1}(\Sigma) \subseteq \Sigma \text {. }
$$


But then, the fact that $H[g][\bar{h}]=\phi\left[N_{\tau}, \pi^{-1}(\Sigma)\right]$ yields a $\Sigma^{g}$-iteration of $N$ which fails to move one of the term relations for $A$ correctly. This is a contradiction.

Motivated by these claims, working in $V[g][h]$ we put for $x \in \mathbb{R}$ and $A \in \mathcal{A}$,

$$
\begin{aligned}
& x \in A^{h} \Leftrightarrow \quad \exists i \exists \delta \exists l\left(i: N_{\tau} \rightarrow P \text { is a } \sum\right. \text {-iteration and } \\
&\left.l \text { is } P[g] \text {-generic and } x \in i\left(\sigma_{A, \delta}\right)_{g \times l}\right) .
\end{aligned}
$$

It is easy to see that

$$
A^{h} \cap V[g]=A,
$$

because the iteration given by 67 can be taken in $H\left(\omega_{2}\right)^{V}$ in this case, and such iterations correspond to iterations by $\Sigma^{g}$, which moves $\tau_{A, \delta}^{N}$ correctly. Note $\mathcal{A}$ is closed under real quantification. Fixing $i$, we have a $j$ such that

$$
V[g] \models \forall \vec{x} \in \mathbb{R}^{<\omega}\left(A_{j}(\vec{x}) \Leftrightarrow \exists y A_{i}(\vec{x}, y)\right) .
$$

But this fact is coded into the first order theory over $N_{\tau}$ of the term relations $\sigma_{A_{i}, \delta}$ and $\sigma_{A_{j}, \mu}$. More precisely, given $\delta<\mu$ Woodins of $N_{\tau}$, there is a $p \in g$ which forces over $N_{\tau}$ the statement "whenever $k$ is $N_{\tau}[\dot{g}]$-generic over $\operatorname{Col}(\omega, \delta)$ and $\vec{x} \in N_{\tau}[\dot{g}][k]$, then $\vec{x} \in\left(\sigma_{A_{j}, \delta}\right)_{\dot{g} \times k}$ if and only if 1 forces in $\operatorname{Col}(\omega, \mu)$ over $N_{\tau}[\dot{g}][k]$ " there is a $y$ such that $(\vec{x}, y) \in\left(\sigma_{A_{i}, \mu}\right)_{\dot{g} \times t}$, where $t$ is the re-arrangement of $k \times \dot{G}$." " These first order facts are preserved by our genericity iterations of $N_{\tau}$, and those are sufficiently numerous by 67 , and coherent in how they move the $\sigma_{A, \nu}$ by 68 , that we get

$$
V[g][h] \models \forall \vec{x} \in \mathbb{R}^{<\omega}\left(A_{j}^{h}(\vec{x}) \Leftrightarrow \exists y A_{i}^{h}(\vec{x}, y)\right) .
$$

We leave any further calculation here to the reader. ${ }^{10}$ Also, for any $i$ there is a $j$ such that

$$
V[g] \models \forall \vec{x}\left(A_{i}(\vec{x}) \Leftrightarrow \neg A_{j}(\vec{x})\right) .
$$

Fixing such $i, j$, we then have

$$
V[g][h] \models \forall \vec{x}\left(A_{i}^{h}(\vec{x}) \Leftrightarrow \neg A_{j}^{h}(\vec{x})\right) .
$$

Generalizing slightly, we get that for any formula $\phi$ in the language of our two structures, there is a $j=j_{\phi}$ such that for all $\vec{x}$ in $V[g]$,

$$
\left(\left(\mathrm{HC}^{V[g]}, \in, A_{i}\right)_{i<\omega} \models \phi[\vec{x}]\right) \Leftrightarrow A_{j}(\vec{x}),
$$

\footnotetext{
${ }^{10}$ See [21] for a similar argument. It was to make this argument possible that we moved to an $N$ with $\omega$ Woodins, rather than just one.
} 
and for all $\vec{x}$ in $V[g][h]$

$$
\left(\left(\mathrm{HC}^{V[g][h]}, \in, A_{i}^{h}\right)_{i<\omega} \models \phi[\vec{x}]\right) \Leftrightarrow A_{j}^{*}(\vec{x}) .
$$

Since $A_{j}=A_{j}^{h} \cap V[g]$, we are done with part (1). Part (2) of the theorem follows easily from part (1), and the fact that the $A_{i}$ code the appropriate fragments of the theory of $J_{\beta}(\mathbb{R})$. It is routine then use the $A_{i}^{h}$ to construct a structure over $\mathbb{R} \cap V[g][h]$ into which $J_{\beta}(\mathbb{R})^{V[g]}$ embeds with the required degree of elementarity. One need only show the structure over $\mathbb{R} \cap V[g][h]$ one gets is well-founded. The proof of this is a reflection argument very similar to the proof of Claim 55, so we omit it. We let $\beta^{h}$ be such that $\omega \beta^{h}$ is the height of this structure.

Finally, we turn to (3). By part (2), $\beta^{h}$ ends a gap $\left[\alpha^{h}, \beta^{h}\right]$ in $V[g][h]$, and the $A_{i}^{h}$ constitute a self-justifying system which seals this gap. We claim that the $A_{i}^{h}$ guide an iteration strategy $\Sigma^{h}$ for $N_{\tau}$, or equivalently for $N=N_{\tau}[g]$, and that $\Sigma^{g} \cup \Sigma \subseteq \Sigma^{g, h}$. This is again a simple reflection argument along the lines of the proof of Claim 55, and so again, we omit it. Being guided by a self-justifying system, $\Sigma^{h}$ condenses well.

Remark 69. An earlier version of this paper, posted on the first author's webpage, had at this point an argument purporting to show that the pullback strategy $i(\Sigma)^{i}$ used in the proof of Lemma 64 codes that same sort of gap in the $L(\mathbb{R})$ of $\operatorname{Ult}(V, G)$ that $\Sigma^{G}$ does in $V[g][G]$. Trevor Wilson found a serious gap in this argument, namely, its assumption that the theory coded into $i(\Sigma)^{i}$ describes a wellfounded model. Fortunately, we don't need this argument.

Remark 70 . We eventually get $\beta^{G}=\beta$, but only after we have shown $W_{\gamma+1}^{*}$ holds in $V[g]$. That is because the Foreman-Magidor argument requires a universally Baire prewellorder of length $\beta$ in $V[g]$.

Corollary 71. Let $G \subset\left(P\left(\omega_{1}\right) / N S\right)^{V}$ be generic over $V[g]$. Then

$$
V[g][G] \models \forall A \in H\left(\omega_{1}\right) \forall n\left(P_{n}^{\Sigma^{G}}(A)^{\sharp} \text { exists and is } \omega_{1} \text {-iterable }\right) .
$$

Proof. Suppose first that $\alpha \in \operatorname{ran} \pi_{G}$. We may then suppose that $N_{\tau}=$ $N$ is in $V$, and countable there. We can then repeat the inductive proof of theorem 28, showing that $H\left(\omega_{3}\right)^{V}$ is closed under the $P_{n}^{\Sigma}$ operator, for all $n$. This easily yields the corollary.

Suppose next that $\alpha \notin \operatorname{ran}\left(\pi_{G}\right)$, and let $\alpha_{0}$ be least such that $\alpha<$ $\pi_{G}\left(\alpha_{0}\right)$. As in the inadmissible case, $\alpha_{0}$ begins a gap in $V$. Thus $\pi_{G}\left(\alpha_{0}\right)$ begins a gap in $\operatorname{Ult}(V, G)$, so $\beta^{G}<\pi\left(\alpha_{0}\right)$. It follows that in $\operatorname{Ult}(V, G), J_{\beta^{G}+\omega}(\mathbb{R}) \models$ AD. Standard results on the existence of iterable models with Woodin cardinal under determinacy imply that $J_{\beta^{G}+\omega}(\mathbb{R})^{V[G]} \models \forall A \in H\left(\omega_{1}\right) \forall n\left(P_{n}^{\Sigma^{G}}(A)^{\sharp}\right.$ exists and is $\omega_{1}$-iterable ). ( 



by the elementarity of the Cohen ultrapower.

Now let $h$ be $\operatorname{Col}\left(\omega, \omega_{2}^{V}\right)$-generic over $V[g]$, and $G \in V[g][h]$ be $N S$ generic over $V[g]$. Note that the extension from $V[g][G]$ to $V[g][h]$ is by a partial order which, in $V[g][G]$, is of size $\omega_{1}$ and collapses $\omega_{1}$. So $V[g][g]$-to- $V[g][h]$ is a homogeneous extension. We shall show the mice $P_{n}^{\Sigma^{G}}(A)^{\sharp}$ given by 71 are definable from $A$ in $V[g][h]$, thus in $V[g]$ when $A \in V[g]$. Definability comes from lifting their strategies to $V[g][h]$, and that comes from lifting the operators themselves to $V[g][[h]$. To do that, we need to use simultaneous reflection in $V$, so we must consider the $P_{n}^{\Sigma}$-sharp operators on $H\left(\omega_{3}\right)^{V}$. The following lemma does the job.

Lemma 72. For all $n<\omega$,

(1) $V \models$ for all transitive $A \in H\left(\omega_{3}\right)$ such that $N_{\tau} \in A, P_{n}^{\Sigma}(A)^{\sharp}$ exists and is $\omega_{3}$-iterable,

(2) if $A \in H\left(\omega_{3}\right)^{V}$ and $P$ is such that $V \models " P=P_{n}^{\Sigma}(A)^{\#}$ is $\omega_{3}$ iterable", then $V[g][h]=" P=P_{n}^{\Sigma^{h}}(A)^{\sharp}$ is $\omega_{1}$-iterable", and

(3) $V[g][h] \models$ for all countable transitive $A$ such that $N \in A$, $P_{n}^{\Sigma^{h}}(A)$ exists and is $\omega_{1}$-iterable.

Proof. By induction on $n$. We have already proved (1) when $n=0$. Part (2) is trivial in this case, since the iterations of $P$ are all linear iterations by its unique extender, and hence are all in $V$. For part (3), Note that the $P_{0}^{\Sigma}$-sharp operator determines itself on $V[g][h]$. More precisely, the $P_{0}^{\Sigma}$-sharp operator on $H\left(\omega_{3}\right)^{V}$ determines the $P_{0}^{\Sigma^{h}}$-sharp operator on $H\left(\omega_{1}\right)^{V[g][h]}$. For let $A$ be countable transitive in $V[g][h]$, and say $A=\rho_{g \times h}$. Let $B \in V$ be the transitive closure of $\left\{\rho, N_{\tau}\right\}$. We have an $\omega_{3}^{V}$-iterable $P=P_{0}^{\Sigma}(B)^{\sharp}$ in $V$. But then $P[g \times h]$ exists in $V[g][h]$, and we can obtain $P_{0}^{\Sigma^{h}}(A)^{\sharp}$ from it. This is because the determination of $\Sigma^{h}$ from $\Sigma$ we gave (via $\mathbb{R}$-genericity iterations which create a self-justifying system guiding $\Sigma^{h}$ ) is sufficiently local that if $M \models \mathrm{ZFC}$ and $\Sigma \cap M \in M$ and $g, h \in M$, then $\sigma^{h} \cap M \in M$ and is uniformly-in- $M$ definable over $M$ from $\Sigma \cap M, g$, and $h$. Iterations of $P_{0}^{\Sigma^{h}}(A)^{\sharp}$ correspond to iterations of $P$ as in (2). The latter stretch $\Sigma$ into $\Sigma$, so the former stretch $\Sigma^{h}$ into $\Sigma^{h}$.

Now suppose (1) through (3) hold for $n=k$. We consider (1) for $n=k+1$. We first consider the case $A \in H\left(\omega_{2}\right)^{V}$. In $V[g]$, let $B$ be the first admissible set over $\{A, g\}$, so that $N \in B$. By Corollary 71 we have $P$ in $V[g][G]$ such that

$$
V[g][G] \models P=P_{k+1}^{\Sigma^{G}}(B)^{\sharp},
$$


in the sense that $P$ has the first order properties, and is $\omega_{1}^{V[g][G]}$-iterable via a strategy which moves $\Sigma^{G}$ to itself. We claim that

$$
V[g][h] \models P=P_{k+1}^{\Sigma^{h}}(B)^{\sharp},
$$

in the sense that $P$ is $\omega_{1}^{V[g][h]}$ iterable in $V[g][h]$ via a strategy which moves $\Sigma^{h}$ to itself. The iteration strategy for $P$ in $V[g][h]$ is the one guided by the $Q$-structures provided by (3) for $n=k$. Let $\Gamma$ be this strategy, and suppose $\Gamma$ fails in $V[g][h]$. Let $\mathcal{O}^{h}$ be the $P_{k}^{\Sigma^{h}}$-sharp operator of $V[g][h]$, and let $\mathcal{O}=\mathcal{O}^{h} \uparrow V[g][G]$. So $\mathcal{O}$ is defined on $A \in H\left(\omega_{2}\right)^{V[g][G]}$ with $N \in A$. We have that $\mathcal{O} \in V[g][G]$, because the extension to $V[g][h]$ is homogeneous, and $\mathcal{O}^{h}$ is definable in $V[g][h]$ from $\Sigma$. Moreover, $\mathcal{O}$ determines the full $\mathcal{O}^{h}$ in $V[g][h]$ via the process we have described. So $\Gamma$ is definable in $V[g][h]$ from $\mathcal{O}$, and

$V[g][G] \models$ "it is forced that the strategy for $P$ determined by $\mathcal{O}$ fails".

From the point of view of $V[g][G]$, the forcing in question is just $\operatorname{Col}\left(\omega, \omega_{1}\right)$. But now, working in $V[g][G]$, let

$$
\pi: S \rightarrow V_{\eta}
$$

where $S$ is countable transitive, with everything relevant in its range. Let $l$ be $S$-generic for the collapse of $\omega_{1}^{S}$, with $l \in V[g][G]$. It is then easy to see that $\pi^{-1}(\mathcal{O})$ is contained in the $P_{k}^{\Sigma^{G}}$-sharp operator of $V[g][G]$, and what it determines on $S[l]$ is also contained in the $P_{k}^{\Sigma^{G}}$ sharp operator of $V[g][G]$. Since $P$ did have a strategy in $V[g][G]$ guided by this operator, we have a contradiction, proving our claim.

Now we can invert the extension leading from $A$ to $B$, getting a $\Sigma^{h}$ premouse $Q$ over $A$ such that

$$
P=\text { canonical re-arrangement of } Q[g] \text { as a premouse over } B \text {. }
$$

By the homogeneity of the forcing and the definablity of $P$ in $V[g][h]$, we get that inductively that all levels of $Q$ are in $V$, and that all trees to which $\Sigma^{h}$ is applied in such levels are in $V$. Thus $Q$ is a $\Sigma$-premouse in $V$. The iteration strategy for $P$ in $V[g][h]$ induces a strategy for $Q$ in $V$, and this strategy is in $V$ by homogeneity, and it witnesses

$$
Q=P_{k+1}^{\Sigma}(A)^{\sharp}
$$

in $V$, and that $Q$ is $\omega_{3}$-iterable in $V$. We now use simultaneous reflection to extend the $P_{k+1}^{\Sigma}$-sharp operator to $H\left(\omega_{3}\right)$ in $V$, just as we did in the $n=0$ case. For $A \subseteq \omega_{2}$, the key definition is

$$
\begin{aligned}
(\phi, \vec{\alpha}) \in P_{k+1}^{\Sigma}(A)^{\sharp} \Leftrightarrow \quad & \text { for club many } \sigma \in P_{\omega_{1}}\left(H_{\omega_{2}}\right) \\
& \left(\phi, \vec{\alpha}_{\sigma}\right) \in P_{k+1}^{\Sigma^{\pi_{\sigma}}}\left(A_{\sigma}\right)^{\sharp} .
\end{aligned}
$$


Here we use Lemma 49 to see that for each such $\sigma \in P_{\omega_{1}}\left(\omega_{2}\right), P_{k+1}^{\Sigma^{\pi_{\sigma}}}\left(A_{\sigma}\right)^{\sharp}$ exists, and is $\omega_{3}$-iterable. Just as in the $n=0$ case, we get that everything is decided on a club, so that the definition yields a structure with the first order properties of $P_{k+1}^{\Sigma}(A)^{\sharp}$. An argument parallel to that in the $n=0$ case shows that this structure interprets $\dot{\Sigma}$ as $\Sigma$, and that it is $\omega_{3}$-iterable in a way that moves $\Sigma$ to itself. Here one uses the corresponding properties of the $P_{k+1}^{\Sigma^{\pi_{\sigma}}}\left(A_{\sigma}\right)^{\sharp}$, and the fact that $\Sigma$ collapses into its pullbacks under Skolem hulls. This finishes the proof of (1) at $k+1$. We leave the straightforward proofs of (2) and (3) at $k+1$ to the reader.

We have finally done what we set out to do in this section.

Lemma 73. The following holds in $V[g]$. For all transitive $A \in H\left(\omega_{1}\right)$ such that $N \in A$, and all $n<\omega, P_{n}^{\Sigma^{g}}(A)^{\sharp}$ exists and is $\omega_{1}$-iterable. Hence $W_{\gamma+1}^{*}$ holds in $V[g]$.

Proof. This follows at once from (3) of 72 , and the homogeneity of $\operatorname{Col}\left(\omega, \omega_{1}\right)$. Every set in $J_{\gamma}(\mathbb{R})^{V[g]}$ is (boldface) projective in $\Sigma^{g}$. So the $P_{n}^{\Sigma^{g}}(A)^{\sharp}$ are the desired coarse capturing mice.

Lemma 74. $I_{\gamma+1}$ holds.

Proof. We have shown that the $P_{n}^{\Sigma^{G}}$-sharp operator of $V[g][G]$, when restricted to $\mathrm{HC}^{V[g]}$, is just the $P_{n}^{\Sigma^{g}}$-sharp operator of $V[g]$. Projectivein- $\Sigma$ truth is coded into these operators, so we get

$$
\left(\mathrm{HC}^{V[g]}, \in, \Sigma^{g}\right) \prec\left(\mathrm{HC}^{V[g][G]}, \in, \Sigma^{G}\right) .
$$

But $\Sigma^{g}$ codes truth at the bottom of the Levy hierarchy over $J_{\gamma}(\mathbb{R})^{V[g]}$, and $\Sigma^{G}$ codes truth at the bottom of the Levy hierarchy over $J_{\gamma^{G}}(\mathbb{R})^{V[g][G]}$, where $\gamma^{G}=\beta^{G}$ if our gap was weak, and $\gamma^{G}=\beta^{G}+1$ otherwise. (Truth is coded via $\mathbb{R}$-genericity iterations which determine self-justifying systems at the end of these gaps, as in our argument.) So we get from the line displayed above an embedding

$$
\pi: J_{\gamma+1}(\mathbb{R})^{V[g]} \rightarrow J_{\gamma^{G}+1}(\mathbb{R})^{V[g][G]}
$$

which is $\Sigma_{1}$ elementary. But in $V[g]$ we have an $\omega_{1}$ Universally Baire prewellorder of length $\gamma$, so we can use the Foreman-Magidor argument to show $\gamma=\gamma^{G}$, and $\pi=$ identity.

Repeating the relevant arguments $\omega$ times gives $W_{\gamma+\omega}^{*}$ in $V[g]$ as well as $I_{\gamma+\omega}$. 


\section{Concluding Remarks}

Like many of the well-known consequences of $\mathrm{MM}(c)$, our hypotheses follow from the Strong Reflection Principle of Todorcevic, denoted $\operatorname{SRP}\left(\omega_{2}\right)$, which asserts that for every projective stationary subset $S$ of $\left[\omega_{2}\right]^{\omega}$ there is an ordinal $\delta<\omega_{2}$ so that $S \cap[\delta]^{\omega}$ contains a club in $[\delta]^{\omega}$. Thus, our main theorem gives $\operatorname{AD}^{L(\mathbb{R})}$ from $\operatorname{SRP}\left(\omega_{2}\right)$ as well. While this represents the best known lower bound for the strength of $\operatorname{SRP}\left(\omega_{2}\right)$, and even $\operatorname{MM}(c)$, these principles are almost certainly much stronger. ${ }^{11}$ Moreover, in a precise sense, our arguments can not take us much farther. In section 9.5 of [25] Woodin defines principles $\operatorname{SRP}^{*}\left(\omega_{2}\right)$ and $\operatorname{WRP}_{(2)}^{*}\left(\omega_{2}\right)$ and shows that the latter is a consequence of the former if $N S$ is saturated (see Lemma 9.93 of [25]). $\operatorname{SRP}^{*}\left(\omega_{2}\right)$ asserts the existence of a normal fine ideal $I \subset P\left(\left[\omega_{2}\right]^{\omega}\right)$ with the following two properties: (1) For every $T \in P\left(\omega_{1}\right) \backslash N S$ the set

$$
S_{T}=\left\{\sigma \in\left[\omega_{2}\right]^{\omega} \mid \sigma \cap \omega_{1} \in T\right\}
$$

is $I$-positive, and (2) for every $S \subset\left[\omega_{2}\right]^{\omega}$ which satisfies $S \cap S_{T} \notin I$ for every $T \in P\left(\omega_{1}\right) \backslash N S$, there is $\gamma<\omega_{2}$ such that $S \cap[\gamma]^{\omega}$ contains a club in $[\gamma]^{\omega}$. WRP $_{(2)}^{*}\left(\omega_{2}\right)$ asserts the existence of a normal fine ideal $I$ with property (1) above so that any pair $S, T \notin I$ simultaneously reflect to stationary sets in some $[\gamma]^{\omega}$. Woodin obtains $S R P^{*}\left(\omega_{2}\right)$ together with the saturation of $N S$ in a $\mathbb{P}_{\max }$ extension of a determinacy model whose existence is equiconsistent with $\omega^{2}$ Woodin cardinals.

Theorem 75. (Woodin; 9.102 of [25]) The following are equiconsistent.

(1) $F \cap L(F, \mathbb{R})$ is an ultrafilter where $F$ is the club filter on $[\mathbb{R}]^{\omega}$ and $A D$ holds in the model $L(F, \mathbb{R})$

(2) There exists a set of Woodin cardinals of order type $\omega^{2}$

Moreover, if $G$ is $\mathbb{P}_{\max }$ generic over $L(F, \mathbb{R})$ as in (1) then

$$
L(F, \mathbb{R})[G] \models \operatorname{SRP}^{*}\left(\omega_{2}\right) \text { and } N S \text { saturated } .
$$

Woodin remarks in 9.98 of [25] that his proof of Theorem 10 also proves PD from $N S$ saturated and $\mathrm{WRP}_{(2)}^{*}\left(\omega_{2}\right)$. The same is true of our argument.

Corollary 76. Assume NS saturated, $\mathrm{WRP}_{(2)}^{*}\left(\omega_{2}\right)$ holds, and $2^{\omega} \leq \omega_{2}$. Then $L(\mathbb{R}) \models A D$.

\footnotetext{
${ }^{11}$ They can be obtained via forcing from a supercompact cardinal (see [4]) or from $A D_{\mathbb{R}}+\Theta$ regular (see 10.88 of $[25]$ ).
} 
Proof. This amounts to checking that $\mathrm{WRP}_{(2)}^{*}\left(\omega_{2}\right)$ can serve in the place of $\operatorname{WRP}_{(2)}\left(\omega_{2}\right)$ in every $H\left(\omega_{2}\right)$ to $H\left(\omega_{3}\right)$ lifting argument. For example in Lemma 14 we would show that the sets $S_{t}$ are measured by the filter dual to $I$ (as opposed to the club filter). One therefore gets $2^{\omega_{1}}=\omega_{2}$ as in Lemma 8 and the rest of the proof is the same as in the proof of the Main Theorem.

Very likely the $2^{\omega} \leq \omega_{2}$ hypothesis can be dropped, although we haven't thought this through. ${ }^{12}$ Thus the consistency strength of NS saturated together with $\operatorname{WRP}_{(2)}^{*}\left(\omega_{2}\right)$ and $2^{\omega} \leq \omega_{2}$ is somewhere in the interval

$$
\text { ( } \omega \text { Woodins, } \omega^{2} \text { Woodins] }
$$

and we have reason to believe that the following conjecture is true.

Conjecture 77 . The following are equiconsistent.

(1) There exists a set of Woodin cardinals of order type $\omega^{2}$

(2) $N S$ is saturated and $\mathrm{WRP}_{(2)}^{*}\left(\omega_{2}\right)$ holds.

(3) $N S$ is saturated and $\mathrm{SRP}^{*}\left(\omega_{2}\right)$ holds.

The first step is to prove that $K(\mathbb{R}) \models A D$. We leave this for another time.

\section{REFERENCES}

[1] Feng, Q., Jech, T., Projective Stationary Sets and Strong Reflection Principles, Journal of the London Mathematical Society, 58 (2), 271-283, 1999

[2] Feng, Q., Magidor, M., Woodin, H., Universally Baire sets of reals, MSRI Pub. 26, 1992, pp. 203-242

[3] Foreman, M., Magidor, M., Large cardinals and definable counterexamples to the continuum hypothesis, Ann. Pure Appl. Logic 76 (1995), no. 1, 47-97

[4] Foreman, M., Magidor, M., Shelah, S., Martin's Maximum, Saturated Ideals, and Nonregular Ultrafilters, Annals of Mathematics 127 (1998), 1-47

[5] Hauser, K., Generic Relativizations of Fine Structure, Archive for Mathematical Logic 39, 2000, no. 4, 227-251

[6] Jech, T., Stationary Sets, in: Handbook of Set Theory, vol. 1, M. Foreman and A. Kanamori eds., Springer 2010, pp. 93-129.

[7] Kechris, A., Woodin, H., Equivalence of Partition Properties and Determinacy, Proc. Natl. Acad. Sci. USA 80 (1983), 1783-1786.

[8] A.S. Kechris and W.H. Woodin, Generic codes for uncountable ordinals, in: Games, scales, and Suslin cardinals, The Cabal Seminar, volume I, Kechris et. al. eds., Cambridge University Press, 2008, pp. 379-397.

[9] Martin, D., Steel, J., A proof of projective determinacy, J. Amer. Math. Soc. 2 (1989), no. 1, 71-125.

[10] Mitchell, W., Steel, J., Fine Structure and Iteration Trees, Lecture Notes in Logic, Vol. 3, Springer Verlag, 1991

\footnotetext{
${ }^{12}$ The point is that we use $2^{\omega_{1}}=\omega_{2}$ at various points and the hypotheses $N S$ saturated and WRP $_{(2)}^{*}\left(\omega_{2}\right)$ give all of Lemma 8 except Todorcevic's bound on $2^{\omega}$.
} 
[11] Moschovakis, Y., Descriptive Set Theory, Studies in Logic and the Foundations of Mathematics, 100. North-Holland Publishing Co., Amsterdam-New York, 1980

[12] Neeman, I., Optimal proofs of determinacy, Bull. Symbolic Logic 1 (1995), no. $3,327-339$

[13] Neeman, I., Itay Optimal proofs of determinacy II, J. Math. Log. 2 (2002), no. $2,227-258$

[14] R.D. Schindler and J.R. Steel, The self-iterability of $L[E]$, Journal of Symbolic Logic, vol. 74 (2009), pp. 751-779.

[15] Schindler, R., Steel, J., The Strength of AD, unpublished manuscript.

[16] Schindler, R., Steel, J., The Core Model Induction, unpublished manuscript.

[17] Steel, J., Scales on $\Sigma_{1}^{1}$ sets, Cabal seminar 79-81, 72-76, Lecture Notes in Math., 1019, Springer, Berlin, 1983.

[18] Steel, J., The core model iterability problem, Lecture Notes in Logic, 8. Springer-Verlag, Berlin, 1996.

[19] Steel, J., A Theorem of Woodin on Mouse Sets, to appear in: Ordinal definability and recursion theorey: The Cabal seminar, volume III, Kechris et. al. eds., Cambridge University Press.

[20] J.R. Steel, An outline of inner model theory, in Handbook of Set Theory, vol. 3, M. Foreman and A. Kanamori eds., Springer 2010, pp. 1595-1684.

[21] J.R. Steel, Derived models associated to mice, in Computational Prospects of Infinity, Chong et. al. eds., Part 1, Tutorials, pp. 105-193, Lect. Notes Ser. Inst. Math. Sci. Natl. Univ. Singap., 14, World Sci. Publ., Hackensack, NJ, 2008.

[22] Steel, J., PFA implies AD in L(R), J. Symbolic Logic 70 (2005), no. 4, 12551296.

[23] Steel, J., Scales in $L(\mathbb{R})$, Cabal seminar 79-81, 107-156, Lecture Notes in Math., 1019, Springer, Berlin, 1983

[24] J.R. Steel, Scales in $K(\mathbb{R})$, in: Games, scales, and Suslin cardinals, The Cabal seminar, volume I, Kechris et. al. eds., Cambridge University Press, 2008, pp. 176-208.

[25] Woodin, H., The axiom of determinacy, forcing axioms, and the nonstationary ideal, de Gruyter Series in Logic and its Applications, 1. Walter de Gruyter \& Co., Berlin, 1999

[26] Zoble, S., Stationary Reflection and the Determinacy of Inductive Games, Doctoral Dissertation, University of California at Berkeley, 2000

[27] Zoble, S., Stationary reflection and the universal Baire property, Fund. Math. 191 (2006), no. 1, 45-56

Department of Mathematics, University of California at Berkeley

E-mail address: steel@math.berkeley.edu

Department of Mathematics, Wesleyan University

E-mail address: azoble@wesleyan.edu 